\title{
Reduced dynamics and symmetric solutions for globally coupled weakly dissipative oscillators
}

\author{
Peter Ashwin, \\ Department of Mathematical Sciences, \\ University of Exeter, \\ Exeter EX4 4QE, UK
}

\author{
Gerhard Dangelmayr, \\ Department of Mathematics, \\ Colorado State University, \\ Fort Collins, Co 80523, USA
}

October 14,2004

\begin{abstract}
Systems of coupled oscillators may exhibit spontaneous dynamical formation of attracting synchronized clusters with broken symmetry; this can be helpful in modelling various physical processes. Analytical computation of the stability of synchronized cluster states is usually impossible for arbitrary nonlinear oscillators. In this paper we examine a particular class of strongly nonlinear oscillators that are analytically tractable. We examine the effect of isochronicity (a turning point in the dependence of period on energy) of periodic oscillators on clustered states of globally coupled oscillator networks.

We extend previous work on networks of weakly dissipative globally coupled nonlinear Hamiltonian oscillators to give conditions for the existence and stability of certain clustered periodic states under the assumption that dissipation and coupling are small and of similar order. This is verified by numerical simulations on an example system of oscillators that are weakly dissipative perturbations of a planar Hamiltonian oscillator with a quartic potential.

Finally we use the reduced phase-energy model derived from the weakly dissipative case to motivate a new class of phase-energy models that can be usefully employed for understanding effects such as clustering and torus breakup in more general coupled oscillator systems. We see that the property of isochronicity usefully generalizes to such systems, and we examine some examples of their attracting dynamics.
\end{abstract}




\section{Introduction}

Coupled oscillators are often used as dynamical models in many areas of the applied sciences. As model dynamical systems they are very useful in allowing one to explore the dynamics of higher dimensional systems, and their study has a respectable past; see for example [7]. In this paper we consider some aspects of coupled oscillator dynamics and clustering in globally coupled systems of oscillators.

In previous papers $[5,6,10]$ we extended some perturbation methods for strongly nonlinear, weakly dissipative systems of $[20,16,17,8]$ to coupled systems and highlighted the importance of isochronicity, a turning point of the period-energy function. In particular, we examined in [6] the stability of uniform oscillations (also called fully synchronized or in-phase oscillations) for oscillators that are coupled and weakly dissipative, such that the coupling is much weaker than the dissipation. We also identified that passing through isochronicity can force bifurcations to appear within the system.

This paper focuses on several related questions for systems of globally coupled ( $\mathbf{S}_{n}$-symmetric) oscillators.

1. We extend [6] in that we consider dynamics when the order of coupling is comparable to the order of dissipation; this means that we compute solutions and their stabilities in cases where there is no invariant torus for the dynamics.

2. We find conditions for existence and stability not only for uniform (fully synchronized) oscillations as in [10], but also for several other symmetric periodic states. This includes solutions found in [3] in the weak-coupling limit and also some 'out of phase cluster' states that exist for a certain common type of coupling.

3. We find examples of attracting robust heteroclinic cycles in a system of four globally coupled weakly dissipative oscillators.

4. We introduce a new phase-energy model for coupled oscillators in which isochronicity can usefully be defined, even when the system is not a reduction from a weakly dissipative system. For this system we observe also that passage through isochronicity forces bifurcation.

For the remainder of this section we introduce the system of weakly dissipative coupled planar Hamiltonian oscillators we consider, and define three special types of coupling that are of particular interest. We give in Section 1.2 an overview of our results for this system.

Section 2 analyzes a number of uniform $\left(\mathbf{S}_{n}\right)$, clustered, $\left(\mathbf{S}_{m} \times \mathbf{S}_{n-m}\right)$ and rotating wave oscillations $\left(\mathbf{Z}_{n}\right)$ of such systems in the singular limit of approaching zero dissipation. In general the computations are rather laborious and hence some details are relegated to Appendices. Section 3 applies these results to investigate the bifurcations of a system of four globally coupled oscillators, where the individual oscillators are weakly dissipative perturbations of an oscillator with a quartic potential, first studied in the uncoupled case in [9]. We give some simulations illustrating the more complicated attractors that one can find in this system.

Motivated by the reduced models obtained from weakly dissipative oscillators by averaging, in Section 4 we consider a system of coupled phase-energy oscillators that possess a natural concept of isochronicity. These models permit one to investigate the dynamics of coupled oscillators in regimes where a reduction to phase dynamics is not always possible in that the amplitude (or energy) of each oscillator also plays a role. Nonetheless, we still find that isochronicity still plays an important role in organizing bifurcations within this system. 


\subsection{Weakly dissipative, strongly nonlinear oscillators}

We examine systems of weakly dissipative coupled planar Hamiltonian oscillators of the form

$$
\frac{d^{2} v_{i}}{d t^{2}}+U^{\prime}\left(v_{i}\right)=\varepsilon F_{i}
$$

where $U(v)$ is a smooth potential with at least one local minimum, $\varepsilon$ is a small parameter and $F_{i}$ represents the dissipation and coupling between the oscillators. We do not assume that $U$ is quadratic and so in the singular limit $\varepsilon=0$ the oscillator is Hamiltonian but nonlinear. We assume that we can write the dissipation $(p)$ and coupling $(q)$ terms in the form

$$
\begin{aligned}
F_{i} & =f_{i}+\varepsilon g_{i}, \\
f_{i} & =p\left(v_{i}, u_{i}\right)+\sum_{j \neq i} q\left(v_{i}, u_{i}, v_{j}, u_{j}\right), \\
g_{i} & =p_{2}\left(v_{i}, u_{i}\right)+\sum_{j \neq i} q_{2}\left(v_{i}, u_{i}, v_{j}, u_{j}\right),
\end{aligned}
$$

where we write $u_{i}=\frac{d v_{i}}{d t}$. We include the second order terms $g_{i}$ because even in generic cases they can affect certain $O(\varepsilon)$-terms of the oscillating solutions, in particular the frequency corrections (see Section 2).

The coupling we consider is global coupling, also called fully symmetric or all-to-all coupling in the literature, and so the network of oscillators has the symmetries of the full permutation group on $n$ objects, $\mathbf{S}_{n}$. In the limit of $\varepsilon$ small and coupling weaker than dissipation, the oscillators are slaved to their phases and the analysis in [3] can be applied.

We will consider generic cases as well as the following three special types of coupling depending on the functional form of $q$. We say there is:

Coupling via derivatives only (CDO) if the coupling terms $q$ in (3) are independent of $v_{i}$ (i.e. only dependent on $u_{i}$ ).

Coupling via positions only (CPO) if the coupling terms $q$ in (3) are independent of $u_{i}$ (i.e. only dependent on $v_{i}$ ).

Symmetric coupling in the derivatives (SCD) if the coupling function $q\left(v_{i}, u_{i}, v_{j}, u_{j}\right)$ is jointly even in $\left(u_{i}, u_{j}\right)$, i.e.,

$$
q\left(v_{i},-u_{i}, v_{j},-u_{j}\right)=q\left(v_{i}, u_{i}, v_{j}, u_{j}\right)
$$

Note that CDO that is linear and non-trivial cannot be SCD. However, CPO always gives SCD. Furthermore, the conditions for these special forms of coupling only involve the leading order coupling term $q$. It turns out that if $q$ satisfies one of these conditions, this has a strong effect on the existence and stability of certain solutions, see Section 1.2. We do not insist that $q_{2}$ has a special form when analyzing the implications of CDO, CPO and SCD.

We conclude by introducing phase-energy coordinates $\left(\psi_{i}, \alpha_{i}\right)$, which are vital for the analysis of (1). There is no gain in using canonical action variables instead of energies, since ultimately we

are studying a dissipative system. As detailed in [6], the system (1) can be transformed to have the form:

$$
\begin{aligned}
\frac{d \psi_{i}}{d t} & =\omega\left(\alpha_{i}\right)-\varepsilon \omega\left(\alpha_{i}\right) z^{\prime}\left(\psi_{i}, \alpha_{i}\right) F_{i} \\
\frac{d \alpha_{i}}{d t} & =\varepsilon \omega\left(\alpha_{i}\right) \dot{z}\left(\psi_{i}, \alpha_{i}\right) F_{i} .
\end{aligned}
$$


The function $z(\varphi, \alpha)$ is the solution of

$$
\omega^{2} \dot{z}^{2} / 2+U(z)=\alpha \quad(\cdot=\partial / \partial \varphi)
$$

and $\omega(\alpha)$ is chosen so that $z(\varphi+2 \pi, \alpha)=z(\varphi, \alpha)$. Throughout the paper we stipulate that the phase of $z$ is fixed so that $z(\varphi, \alpha)$ is an even function of $\varphi$, and $\ddot{z}(0, \alpha)>0$. We recall from [6] that there is a useful relation between the phase and energy derivatives of $z$ :

$$
\dot{z}(\omega \dot{z})^{\prime}-\omega \ddot{z} z^{\prime}=1 / \omega\left({ }^{\prime}=\partial / \partial \alpha\right) .
$$

\subsection{Overview}

We now give a brief overview of the periodic solutions studied analytically in Section 2. Each of these solutions has a symmetry that is a subgroup of $\mathbf{S}_{n} \times \mathbf{S}^{1}\left(\mathbf{S}^{1}\right.$ is the circle group) acting on the space of periodic functions $\left\{\left(v_{i}(t), u_{i}(t)\right): 1 \leq i \leq n\right\}$ by permutations $\left(\mathbf{S}_{n}\right)$ and phase shifts $\left(\mathbf{S}^{1}\right)$. The symmetry of an oscillating solution is simply the largest subgroup of $\mathbf{S}_{n} \times \mathbf{S}^{1}$ that leaves the solution invariant (see $[13,3])$.

The asymptotic form of an oscillating solution is found by means of a nonlinear version of the method of Poincaré-Lindstedt that extends the perturbation methods of $[7,20,16,17,8]$ for strongly nonlinear, weakly dissipative systems to coupled systems as in $[5,6,10]$. The analysis starts with a solution of the uncoupled system with the desired symmetry properties and applies first order averaging in the energies to give the persistence condition. This is a system of nonlinear equations for the parameters on which the solution of the uncoupled system depends. When a solution of the persistence condition is found the oscillating solution and its frequency can be determined from the first and second order expansion of the phase and energy equations. The solution stabilities can be determined by asymptotic expansion of the eigenvalues of the monodromy matrix; the relevant entries of this matrix are computed by a regular perturbation analysis. In general this computation is straightforward but laborious and hence the details are relegated to appendices.

The solutions we will study analytically can be divided into 'basic solutions' and 'cluster oscillations' (more precisely, two-cluster oscillations). The former are governed by single persistence conditions for the energies; the latter require the solution of two coupled persistence conditions for the energies and phase shifts. To keep the paper at a reasonable length, we restrict the analysis to a representative subset of solutions. In addition to finding branches and stabilities of generically expected solutions, we do find some unexpected and nongeneric solution behaviour for certain special forms of coupling.

The solutions studied in Section 2 are summarized in Table 1. For generic coupling the basic solutions are the uniform oscillation, rotating wave oscillation and out of phase cluster if $n$ is even. These solutions have maximal symmetries and are predicted in [3] purely by virtue of symmetry considerations. In the special case of SCD coupling the degenerate out of phase cluster oscillations are persistent, for more general coupling they only arise as special cases of cluster oscillations (see Section 3.3).

We will distinguish two types of cluster oscillations: cluster oscillations with energy difference of order $O(\varepsilon)$ between the oscillations in the two groups, and cluster oscillations with energy difference of order $O(1)$. The former are studied in detail in Section 2.3. Their persistence condition, equation (25), yields two equations for the persistence energy and the phase shift at order $O(1)$. The eigenvalues of the monodromy matrix are naturally more complicated than for the basic solutions. Persistence conditions and stability coefficients for $O P C$ and $O P C(m)$ are derived in Section 2.4.

If the equation $\omega(\alpha)=\omega(\beta)$ admits other solutions than the trivial solution $\beta=\alpha$ (i.e., the $(1,1, \ldots, 1)$ resonance surface has several 'sheets'), the averaging approach also admits cluster 


\begin{tabular}{l|c|c|c}
\hline Oscillation Type & Symmetry & Typical phases & Section \\
\hline $\begin{array}{l}U O \\
\text { Uniform oscillation }\end{array}$ & $\mathbf{S}_{n}$ & $(\theta, \theta, \cdots, \theta)$ & 2.1 \\
\hline$R W$ & $\mathbf{Z}_{n}$ & $(\theta, \theta+2 \pi / n, \cdots, \theta+2(n-1) \pi / n)$ & 2.2 \\
Rotating wave & $\left(\mathbf{S}_{n / 2}\right)^{2} \times_{s} \mathbf{Z}_{2}$ & $(\theta, \cdots, \theta, \theta+\pi, \cdots, \theta+\pi)$ & 2.4 \\
\hline $\begin{array}{l}O P C(n \text { even) } \\
\text { Out of phase cluster }\end{array}$ & $\mathbf{S}_{m} \times \mathbf{S}_{n-m}$ & $(\theta, \cdots, \theta, \theta+\pi, \cdots, \theta+\pi)$ & 2.4 \\
\hline $\begin{array}{l}O P C(m) \\
\begin{array}{l}\text { Degenerate out of } \\
\text { phase cluster }\end{array}\end{array}$ & & \\
\hline Cluster oscillations & $\mathbf{S}_{m} \times \mathbf{S}_{n-m}$ & $(\theta, \cdots, \theta, \phi, \cdots, \phi)$ & 2.3 \\
\hline
\end{tabular}

Table 1: Names and symmetries of the periodic solutions studied in Section 2 via averaging (see indicated section for details). We write $G \times{ }_{s} H$ to denote a semidirect product of groups $G$ and $H$. Note that solutions $\mathrm{OPC}(m)$ where $2 m \neq n$ only occur in special cases such as symmetric coupling in the derivatives (SCD).

oscillations with energy difference of order $O(1)$ between the oscillations in the two groups. The persistence condition for these solutions is presented in Section 2.4.3, but we do not analyze their stability. Necessary for this type of solution to exist (in the averaging framework) is that there is a point of isochronicity. In fact, the branching of the resonance surface is what causes the bifurcations and stability exchanges of all basic solutions and cluster oscillations when passing through isochronicity. We study some of these bifurcations in Section 3 for our example system.

\section{Persistence and stability of oscillating solutions}

In this section we use averaging (more precisely a nonlinear version of the method of PoincaréLindstedt) to understand the asymptotic location and stability of a number of solutions of the weakly dissipative oscillators (1), generalizing the analysis of [6].

\subsection{Uniform oscillations; symmetry $\mathrm{S}_{n}$}

The simplest case of periodic solutions of (1) is when all oscillators have the same phase and energy at all points in time; we call these the uniform oscillations, fully synchronized or in-phase oscillations.

\subsubsection{Persistence of uniform oscillations}

Uniform oscillations are solutions of (6) with $\psi_{i}, \alpha_{i}$ independent of $i$. Setting $\psi_{i}=\psi, \alpha_{i}=\alpha$, $z_{i}=z$, the system (6) reduces to the two-dimensional system,

$$
\begin{aligned}
\frac{d \psi}{d t} & =\omega(\alpha)-\varepsilon \omega(\alpha) z^{\prime}(\psi, \alpha) F, \\
\frac{d \alpha}{d t} & =\varepsilon \omega(\alpha) \dot{z}(\psi, \alpha) F
\end{aligned}
$$

where $F=f+\varepsilon g$, and

$$
f(v, u)=p(v, u)+(n-1) q(v, u, v, u)
$$




$$
g(v, u)=p_{2}(v, u)+(n-1) q_{2}(v, u, v, u),
$$

with $v=z(\psi, \alpha), u=\omega(\alpha) \dot{z}(\psi, \alpha)$. The asymptotic form of periodic solutions of (8) can be found by means of a nonlinear version of the Poincaré-Lindstedt method as in $[5,6,8,10]$.

We make the ansatz

$$
(\psi(t, \varepsilon), \alpha(t, \varepsilon))=\left(\psi_{r}(\varphi, \varepsilon), \alpha_{r}(\varphi, \varepsilon)\right),
$$

where $\psi_{r}-\varphi$ and $\alpha_{r}$ are $2 \pi$-periodic in $\varphi$ with the following asymptotic expansion in powers of $\varepsilon$,

$$
\begin{aligned}
& \psi_{r}=\varphi+\varepsilon \phi^{(1)}(\varphi)+O\left(\varepsilon^{2}\right) \\
& \alpha_{r}=\alpha^{(0)}+\varepsilon\left(\alpha^{(1)}+\beta^{(1)}(\varphi)\right)+O\left(\varepsilon^{2}\right),
\end{aligned}
$$

and where without loss of generality we require that all $\varphi$-dependent terms in (9) have zero mean. The phase $\varphi$ is related to $t$ by

$$
\frac{d \varphi}{d t}=\Omega(\varepsilon)=\omega\left(\alpha^{(0)}\right)+\varepsilon \Omega^{(1)}+O\left(\varepsilon^{2}\right)
$$

with the first order frequency correction $\Omega^{(1)}$ to be determined.

When $(\psi, \alpha)=\left(\psi_{r}, \alpha_{r}\right)$ is substituted into (8) and the resulting equation is expanded in powers of $\varepsilon$, the equation for $d \alpha / d t$ at order $O(\varepsilon)$ yields $\dot{\beta}^{(1)}=\dot{z} f$, with the arguments $(v, u)$ of $f$ replaced by $(z(\varphi, \alpha), \omega(\alpha) \dot{z}(\varphi, \alpha))$. The condition for $\beta^{(1)}$ to be periodic leads to the persistence condition, ${ }^{1}$

$$
\overline{\dot{z} f}=0 \text {. }
$$

Equation (10) is a nonlinear equation for $\alpha^{(0)}$, and we assume in the following that $\alpha^{(0)}$ is a fixed, non-degenerate solution of this equation. Functions of $(\varphi, \alpha)$ are then evaluated at $\alpha^{(0)}$ if arguments are omitted.

The equation for $d \psi / d t$ at $O(\varepsilon)$ yields

$$
\Omega^{(1)}+\omega \dot{\phi}^{(1)}=\omega^{\prime}\left(\alpha^{(1)}+\beta^{(1)}\right)-\omega z^{\prime} f .
$$

The "solvability condition" $\left(\phi^{(1)}\right.$ be periodic) requires that

$$
\Omega^{(1)}=\omega^{\prime} \alpha^{(1)}-\overline{\omega z^{\prime} f} .
$$

If this relation is satisfied, $\phi^{(1)}$ is obtained by integrating the equation

$$
\dot{\phi}^{(1)}=\left(\overline{z^{\prime} f}-z^{\prime} f\right)+\left(\omega^{\prime} / \omega\right) \beta^{(1)} .
$$

To find the first order mean-energy and frequency corrections we have to examine the equation for $d \alpha / d t$ at $O\left(\varepsilon^{2}\right)$. Let us denote by $\varepsilon^{2} \beta^{(2)}(\varphi)$ the zero-mean, $2 \pi$-periodic correction of $\alpha_{r}$ at second order. Then, by setting the coefficient of $\varepsilon^{2}$ in the expansion of $d \alpha / d t$ equal to zero, we obtain the following equation,

$$
\Omega^{(1)} \dot{\beta}^{(1)}+\omega \dot{\beta}^{(2)}=(\omega \dot{z} f)^{\prime}\left(\alpha^{(1)}+\beta^{(1)}\right)+(\omega \dot{z} f)_{, \varphi} \phi^{(1)}+\omega \dot{z} g
$$

\footnotetext{
${ }^{1}$ Throughout the paper we use the notation

$$
\bar{h}=\frac{1}{2 \pi} \int_{0}^{2 \pi} h(\varphi) d \varphi
$$
}

to denote the average of a $2 \pi$-periodic function $h(\varphi)$. 
which leads to

$$
\omega \overline{(\dot{z} f)^{\prime}} \alpha^{(1)}+\overline{(\omega \dot{z} f)^{\prime} \beta^{(1)}}-\omega \overline{\dot{z} f \dot{\phi}^{(1)}}+\omega \overline{\dot{z} g}=0
$$

as solvability condition for $\beta^{(2)}$. After substituting $\dot{\phi}^{(1)}$ this condition can be rewritten as

$$
\overline{(\dot{z} f)^{\prime}} \alpha^{(1)}+\overline{(\dot{z} f)^{\prime} \beta^{(1)}}+\overline{\dot{z} z^{\prime} f^{2}}+\overline{\dot{z} g}=0,
$$

which determines $\alpha^{(1)}$ and hence $\Omega^{(1)}$ if $\overline{(\dot{z} f)^{\prime}} \neq 0$.

\subsubsection{Stability of uniform oscillations}

We examine the stability of the uniform oscillations to small perturbations by setting $\psi_{i}=\psi_{r}(\varphi)+$ $\phi_{i}, \alpha_{i}=\alpha_{r}(\varphi)+\beta_{i}$ and estimating the eigenvalues of the monodromy matrix in the limit $\varepsilon \rightarrow 0$.

The linearized system for $\varepsilon=0$ is

$$
\frac{d \phi_{i}}{d t}=\omega^{\prime} \beta_{i}, \quad \frac{d \phi_{i}}{d t}=0 \quad \text { or } \quad \omega \dot{\phi}_{i}=\omega^{\prime} \beta_{i}, \omega \dot{\beta}_{i}=0
$$

The solution of the linearized system as a function of $\varphi$ up to $O(1)$ is then,

$$
\phi_{i}=\frac{\omega^{\prime}}{\omega} \beta_{i 0} \varphi+\phi_{i 0}+O(\varepsilon), \quad \beta_{i}=\beta_{i 0}+O(\varepsilon)
$$

where $\left(\phi_{i 0}, \beta_{i 0}\right)$ are the initial values. We extend this to the order $O(\varepsilon)$,

$$
\begin{aligned}
\phi_{i}(\varphi) & =\frac{\omega^{\prime}}{\omega} \beta_{i 0} \varphi+\phi_{i 0}+\varepsilon \phi_{i}^{(1)}(\varphi)+O\left(\varepsilon^{2}\right), \\
\beta_{i}(\varphi) & =\beta_{i 0}+\varepsilon \beta_{i}^{(1)}(\varphi)+O\left(\varepsilon^{2}\right) .
\end{aligned}
$$

Evaluation of this system at $\varphi=2 \pi$ yields the monodromy matrix that sends $\left\{\phi_{i 0}, \beta_{i 0}\right\}$ to $\left\{\phi_{i}(2 \pi), \beta_{i}(2 \pi)\right\}$. Due to the $\mathbf{S}_{n}$ symmetry, the monodromy matrix consists of $2 \times 2$ blocks $B$ on the diagonal blocks and $2 \times 2$ blocks $A$ on all off-diagonal blocks. The eigenvalues of the monodromy matrix are the eigenvalues of

$$
L=B-A \quad((n-1) \text {-fold }), \quad K=B+(n-1) A \quad \text { (simple) } .
$$

The $O(1)$-terms of the matrices $B, A$ and $K, L$ are

$$
B=\left[\begin{array}{cc}
1 & \omega^{\prime} T \\
0 & 1
\end{array}\right]+O(\varepsilon), \quad A=O(\varepsilon), \quad K=B+O(\varepsilon), \quad L=B+O(\varepsilon),
$$

with $T=2 \pi / \omega$ the period.

It can be seen directly from the persistence calculations that the matrix $K$ has the eigenvalue one, and another eigenvalue $\lambda^{(0)}=1+r \varepsilon+O\left(\varepsilon^{2}\right)$, where

$$
r=2 \pi \overline{(\dot{z} f)^{\prime}} .
$$

This eigenvalue describes the stability of the uniform oscillation in the two-dimensional subspace of the $2 n$-dimensional phase space in which all oscillators are identical.

To classify the transverse eigenvalues, we denote by $\varepsilon l_{i j}$ the $O(\varepsilon)$ correction of the $(i j)$-entry of $L$. The characteristic equation for the eigenvalues $\tilde{\lambda}$ of $L-I$, where $I$ is the $2 \times 2$ identity matrix, can be written as

$$
\tilde{\lambda}^{2}-\left[\left(l_{11}+l_{22}\right) \varepsilon+O\left(\varepsilon^{2}\right)\right] \tilde{\lambda}-\omega^{\prime} T l_{21} \varepsilon+O\left(\varepsilon^{2}\right)=0,
$$


thus at leading order the eigenvalues are $\tilde{\lambda}= \pm \sqrt{\omega^{\prime} T l_{21} \varepsilon}+O(\varepsilon)$. If $\omega^{\prime} l_{21}>0$, the eigenvalues are real, and the order $O(\sqrt{\varepsilon})$ is sufficient. If $\omega^{\prime} l_{21}<0$, the $O(\sqrt{\varepsilon})$-terms are imaginary. In this case we need the real part at $O(\varepsilon)$ which is given by $2 \operatorname{Re}(\tilde{\lambda})=\left(l_{11}+l_{22}\right) \varepsilon+O\left(\varepsilon^{2}\right)$. We therefore have to distinguish the following two generic cases for the perturbations $\lambda_{1,2}(\varepsilon)$ of the double eigenvalue $\lambda_{1,2}(0)=1$ of $L(\varepsilon)$ :

(a) If $\omega^{\prime} l_{21}>0$, the eigenvalues are real and given by $\lambda_{1,2}=1 \pm \sqrt{\omega^{\prime} T l_{21} \varepsilon}+O(\varepsilon)$, hence the fully synchronized oscillation is a saddle.

(b) If $\omega^{\prime} l_{21}<0$, the eigenvalues are complex conjugate with $\left|\lambda_{1,2}\right|^{2}=1+l \varepsilon+O\left(\varepsilon^{2}\right)$, where $l=l_{11}+l_{22}-\omega^{\prime} T l_{21}$.

In Appendix A.1.1 we compute the relevant $O(\varepsilon)$-corrections of $A$ and $B$. With these corrections $l_{21}$ and $l$ can be represented by the following averages,

$$
\begin{aligned}
l_{21} & =-2 \pi n \overline{\dot{z}\left(q_{, v_{2}} \dot{z}+q_{, u_{2}} \omega \ddot{z}\right)}, \\
l & =2 \pi \overline{(\dot{z} f)^{\prime}}-n T \overline{q_{, u_{2}}},
\end{aligned}
$$

where the coupling function is considered as function of four generic variables, $q=q\left(v_{1}, u_{1}, v_{2}, u_{2}\right)$, and the subscripts behind the comma denote the partial derivatives evaluated at $v_{1}=v_{2}=z, u_{1}=$ $u_{2}=\omega \dot{z}$. Assuming $r<0$, the expressions (14) allow us to make some observations directly:

- If $l_{21} \neq 0$, then no matter what the sign of $l_{21}$ is, passage through isochronicity induces a transition from a saddle to a (stable or unstable) spiral. Specifically, if $q_{, u_{2}}=0$ (CPO) and $q_{, v_{2}}>0$ (e.g. linear coupling $q=v_{2}$ ), then $l_{21}<0$ and $l>0$, thus if $\omega^{\prime}<0$ we find a saddle and if $\omega^{\prime}>0$ we find a stable spiral.

- In general, the spiral in case of $\omega^{\prime} l_{21}<0$ is always stable if $\overline{q_{, u_{2}}}>0$. If $\overline{q_{, u_{2}}}<0$ it can be stable for sufficiently small $n$, but eventually becomes unstable when $n$ increases.

In the case of CDO we have $q_{, v_{1}}=q_{, v_{2}}=0$ and therefore

$$
l_{21}=-2 \pi n \omega \overline{q_{u_{2}} \dot{z} \ddot{z}}=-(2 \pi n / \omega) \overline{\dot{S}}=0,
$$

where

$$
S(u)=\int u q_{, u_{2}}(u, u) d u .
$$

Thus the linear stability analysis leads to a degeneracy at $O(\varepsilon)$. To determine the stability of uniform oscillations in this case, we have to go to $O\left(\varepsilon^{2}\right)$ and include quadratic terms in the perturbation analysis.

\subsubsection{Stability of uniform oscillations for CDO coupling}

We assume the case of CDO, i.e. no coupling in the positions at leading order and so $q$ depends only on $\left(u_{1}, u_{2}\right)$. To resolve the problem caused by the degeneracy $l_{21}=0$, we extend the linear stability analysis by including quadratic terms in the equations for the phase and energy perturbations, following the procedure used in [10] for the case of diffusive coupling.

We write the perturbation about the uniform oscillation in the form,

$$
\begin{aligned}
\psi_{i}(t, \varepsilon) & =\psi_{r}(\varphi, \varepsilon)+\varepsilon \phi_{i}(\tau)+\varepsilon^{2}\left(\phi_{i}^{(2)}(\tau)+\psi_{i}^{(2)}(\varphi, \tau)\right)+O\left(\varepsilon^{3}\right), \\
\alpha_{i}(t, \varepsilon) & =\alpha_{r}(\varphi, \varepsilon)+\varepsilon^{2}\left(e_{i}(\tau)+\beta_{i}^{(2)}(\varphi, \tau)\right)+\varepsilon^{3} \beta_{i}^{(3)}(\varphi, \tau)+O\left(\varepsilon^{4}\right),
\end{aligned}
$$


where $\tau=\varepsilon t$ is a slow time and $\psi_{i}^{(2)}$ and $\beta_{i}^{(2)}$ have zero mean. In Appendix A.1.2 we show that the conditions for $\psi_{i}^{(2)}, \beta_{i}^{(3)}$ to be periodic in $\varphi$ lead to the following closed system of equations for the slowly varying functions $\phi_{i}(\tau), e_{i}(\tau)$ :

$$
\begin{aligned}
\frac{d \phi_{i}}{d \tau} & =\omega^{\prime} e_{i}+M \sum_{j}\left(\phi_{j}-\phi_{i}\right), \\
\frac{d e_{i}}{d \tau} & =D e_{i}+N \sum_{j}\left(e_{j}-e_{i}\right)+P \sum_{j}\left(\phi_{j}-\phi_{i}\right)+Q \sum_{j}\left(\phi_{j}-\phi_{i}\right)^{2},
\end{aligned}
$$

where

$$
D=\omega \overline{(\dot{z} f)^{\prime}}, \quad M=-\omega^{2} \overline{z^{\prime} \ddot{z} q_{, u_{2}}}, \quad N=\overline{S^{\prime}}=\overline{q_{, u_{2}}}-M
$$

and

$$
\begin{aligned}
& P=\overline{\left(\dot{z} f^{\prime}-z^{\prime} \dot{f}\right) S}-\overline{(\dot{z} f)^{\prime}} \bar{S}-\left(n \omega^{\prime} / \omega^{2}\right)\left(\overline{S^{2}}-\bar{S}^{2}\right)-(n / \omega)\left(\overline{S S^{\prime}}-\bar{S} \overline{S^{\prime}}\right)+\omega \overline{\dot{z}^{2} q_{2, v_{2}}}+\omega^{2} \overline{\dot{z} \ddot{z} q_{2, u_{2}}}, \\
& Q=\left(\omega^{2} / 2\right) \overline{\dot{z} \dddot{z} q_{, u_{2}}}+\left(\omega^{3} / 2\right) \overline{\dot{z} \ddot{z}^{2} q_{, u_{2} u_{2}}} \text {. }
\end{aligned}
$$

We note that only in the case of CDO coupling the expansion (15) leads to the quadratic system (16). In the general case it is not possible to find a closed system for the $\left(\phi_{i}, e_{i}\right)$. We also note that the term in $P$ defined through $q_{2}$ vanishes if $q_{2}$ depends on the derivatives only, too.

In Appendix A.1.3 we analyze the periodic solutions of the system (16) and their stability. Assuming that the uniform oscillation is a stable solution of (8), i.e. $D<0$, the result of this analysis is that the stability of the uniform oscillation against perturbations of the form (15) is fully determined by a $2 \times 2$-matrix $B^{(0)}$, with

$$
\operatorname{Tr} B^{(0)}=D-n(N+M), \quad \operatorname{det} B^{(0)}=n M(D+n N)+n \omega^{\prime} P .
$$

Moreover, it turns out that in an $O(\varepsilon)$-neighbourhood of the fully synchronized oscillation there exist all types of cluster oscillations consisting of two fully synchronized groups of $m$ and $n-m$ oscillators, respectively, with $n \neq 2 m$. These cluster oscillations are all saddles and the energy difference between the two oscillations is of order $O\left(\varepsilon^{2}\right)$, whereas the phase shift is of order $O(\varepsilon)$. In Section 2.3 we will study the persistence and stability of cluster oscillations with energy difference of order $O(\varepsilon)$ and phase shift of order one for general couplings.

\subsection{Rotating wave oscillations; symmetry $\mathrm{Z}_{n}$}

Rotating wave oscillations with symmetry $\mathbf{Z}_{n}$ are oscillating solutions of (1) for which all oscillators have identical wave forms, with a phase shift of

$$
\vartheta=2 \pi / n
$$

between oscillators $i$ and $i+k$ ( $k$ and $n$ relatively prime). Without loss of generality we set $k=1$. We do not consider the more general case of oscillations with symmetry $\mathbf{S}_{k} \times_{s} \mathbf{Z}_{m}$ where $k m=n$ and $1<k<n[3]$; this could be done by continuing the analysis along the same lines.

\subsubsection{Persistence of rotating waves}

The persistence analysis for rotating wave oscillations proceeds in the same manner as for uniform oscillations. The appropriate Poincaré-Lindstedt ansatz is:

$$
\begin{aligned}
& \psi_{i}=\varphi+i \vartheta+\varepsilon \phi^{(1)}(\varphi+i \vartheta)+O\left(\varepsilon^{2}\right) \\
& \alpha_{i}=\alpha^{(0)}+\varepsilon\left(\alpha^{(1)}+\beta^{(1)}(\varphi+i \vartheta)\right)+O\left(\varepsilon^{2}\right)
\end{aligned}
$$


where $d \varphi / d t=\Omega(\varepsilon)$ as in Section 2.1.1. For $0 \leq i \leq n-1$ we set $z_{i}=z(\varphi+i \vartheta), v_{i}=z_{i}, u_{i}=\omega \dot{z}_{i}$, and omit the subscript if $i=0$. The persistence condition for a solution to (6) in the form of (17) follows again from the solvability condition for $\phi^{(1)}$, which leads here to

$$
\overline{\dot{z} f_{\vartheta}}=0,
$$

where

$$
f_{\vartheta}=p(v, u)+\sum_{i=1}^{n-1} q\left(v, u, v_{i}, u_{i}\right) .
$$

The first order corrections $\Omega^{(1)}$ and $\alpha^{(1)}$ are given by the same expressions as in Section 2.1.1, equations (11) and (12), with $f$ and $g$ replaced by $f_{\vartheta}$ and $g_{\vartheta}$, where $g_{\vartheta}$ is defined analogously to $f_{\vartheta}$ in terms of the second order dissipation and coupling terms.

\subsubsection{Stability of rotating waves}

The $\mathbf{Z}_{n}$-symmetry induces a circulant structure in the monodromy matrix $M$. Writing the first two rows of $M$ in the form

$$
A=\left[A^{(0)}, A^{(1)}, \ldots, A^{(n-1)}\right]
$$

with $2 \times 2$-matrices $A^{(j)}, 0 \leq j \leq n-1$, the rows below the first two are obtained by successive cyclic permutations of the matrix row $A$ to the right. The eigenvalues of $M$ are the eigenvalues of the components $L^{(k)}, 0 \leq k \leq n-1$, of the discrete Fourier transform of $A$, i.e.

$$
L^{(k)}=\sum_{j=0}^{n-1} \rho^{j k} A^{(j)},
$$

where $\rho=e^{i \vartheta}$ is the $n$th primitive root of unity. Since the $A^{(j)}$ are real, $L^{(0)}$ is real, and $L^{(n-k)}$ is the complex conjugate of $L^{(k)}$ for $1 \leq k \leq n-1$.

The leading term of $A^{(0)}$ is again given by

$$
A^{(0)}=\left[\begin{array}{cc}
1 & \omega^{\prime} T \\
0 & 1
\end{array}\right]+O(\varepsilon),
$$

and $A^{(j)}=O(\varepsilon)$ for $1 \leq j \leq n-1$, hence

$$
L^{(k)}=\left[\begin{array}{cc}
1 & \omega^{\prime} T \\
0 & 1
\end{array}\right]+\varepsilon L^{(k, 1)}+O\left(\varepsilon^{2}\right)
$$

for all $0 \leq k \leq n-1$. We can, therefore, pursue the same kind of analysis as for the uniform oscillation, except that now some of the matrices involved may have complex entries.

The relevant terms are again $l_{21}^{(k)}$ and $l^{(k)}=l_{11}^{(k)}+l_{22}^{(k)}-\omega^{\prime} T l_{21}^{(k)}$, with $l_{\nu \mu}^{(k)}(\nu, \mu=1,2)$ the $O(\varepsilon)-$ entries of $L^{(k, 1)}$. These terms are the discrete Fourier transforms of the vectors $\left[a_{21}^{(0)}, \ldots, a_{21}^{(n-1)}\right]$ and $\left[a^{(0)}, \ldots, a^{(n-1)}\right]$, where $a^{(j)}=a_{11}^{(j)}+a_{22}^{(j)}-\omega^{\prime} T a_{21}^{(j)}$ and the $a_{\nu \mu}^{(j)}$ are the entries of the $O(\varepsilon)_{-}$ corrections of the matrices $A^{(j)}$. A completely analogous calculation as for the uniform oscillation yields the following representation of $a_{21}^{(j)}$ and $a^{(j)}$ through averages:

$$
\begin{array}{ll}
a_{21}^{(0)}=-2 \pi \sum_{i=1}^{n-1} \overline{\dot{z} d_{i}}, & a^{(0)}=2 \pi \overline{\left(\dot{z} f_{\vartheta}\right)^{\prime}}+2 \pi \sum_{i=1}^{n-1}\left(\overline{z^{\prime} d_{i}}-\overline{\dot{z} e_{i}}\right), \\
a_{21}^{(j)}=2 \pi \overline{\dot{z} d_{j}}, & a^{(j)}=2 \pi\left(\overline{\dot{z} e_{j}}-\overline{z^{\prime} d_{j}}\right),
\end{array}
$$


where we have set

$$
d_{i}=q_{, v_{2}} \dot{z}_{i}+q_{, u_{2}} \omega \ddot{z}_{i}, \quad e_{i}=q_{, v_{2}} z_{i}^{\prime}+q_{, u_{2}}\left(\omega \dot{z}_{i}\right)^{\prime},
$$

with the arguments $\left(v_{1}, u_{1}, v_{2}, u_{2}\right)$ of $q$ evaluated at $\left(v, u, v_{i}, u_{i}\right)$.

The matrix $L^{(0)}$ is real and has the eigenvalue one. The other eigenvalue is $\lambda^{(0)}=1+r \varepsilon+O\left(\varepsilon^{2}\right)$, where

$$
r=2 \pi \overline{\left(\dot{z} f_{\vartheta}\right)^{\prime}} .
$$

This eigenvalue describes the stability of the rotating wave against perturbations of the same form. The eigenvalues $\lambda_{ \pm}^{(k)}$ of $L^{(k)}$ for $1 \leq k \leq n-1$ are characterized by $l_{21}^{(k)}$ and $l^{(k)}$ similarly as in Section 2.1.2, but now these terms may be complex. A simple calculation shows that these eigenvalues can be characterized as follows:

- If $l_{21}^{(k)}$ is real and $\omega^{\prime} l_{21}^{(k)}<0$, then $\left|\lambda_{ \pm}^{(k)}\right|^{2}=1+\operatorname{Re}\left(l^{(k)}\right) \varepsilon+O\left(\varepsilon^{3 / 2}\right)$.

- In all other cases let $l_{21}^{(k)}=r_{k} e^{i \theta_{k}}$. Then we have $\left|\lambda_{ \pm}^{(k)}\right|^{2}=1 \pm 2 q_{k} \sqrt{\left|\omega^{\prime} T\right| r_{k} \varepsilon}+O(\varepsilon)$, where $q_{k}=\cos \theta_{k} / 2$ if $\omega^{\prime}>0$, and $q_{k}=-\sin \theta_{k} / 2$ if $\omega^{\prime}<0$.

Hence the real and imaginary parts are

$$
\begin{aligned}
& l_{21}^{(k)}=2 \pi b_{21}^{(k)}+2 \pi \sum_{j=1}^{m}\left(c_{21}^{(j+)}(\cos k j \vartheta-1)+i c_{21}^{(j-)} \sin k j \vartheta\right), \\
& l^{(k)}=2 \pi \overline{\left(\dot{z} f_{\vartheta}\right)^{\prime}}+2 \pi b^{(k)}+2 \pi \sum_{j=1}^{m}\left(c^{(j+)}(\cos k j \vartheta-1)+i c^{(j-)} \sin k j \vartheta\right),
\end{aligned}
$$

where $b_{21}^{(k)}=b^{(k)}=0$ and $m=(n-1) / 2$ if $n$ is odd,

$$
b_{21}^{(k)}=\left((-1)^{k}-1\right) \overline{\dot{z} d_{n / 2}}, \quad b^{(k)}=\left((-1)^{k}-1\right)\left(\overline{\dot{z} e_{n / 2}}-\overline{z^{\prime} d_{n / 2}}\right),
$$

and $m=n / 2-1$ if $n$ is even, and

$$
c_{21}^{(j \pm)}=\overline{\dot{z} d_{j}} \pm \overline{\dot{z} d_{n-j}}, \quad c^{(j \pm)}=\overline{\dot{z} e_{j}}-\overline{z^{\prime} d_{j}} \pm\left(\overline{\dot{z} e_{n-j}}-\overline{z^{\prime} d_{n-j}}\right) .
$$

For $n \geq 3$ and generic coupling we can expect a nonzero imaginary part of $l_{21}^{(k)}$ for $1 \leq k \leq n-1$, $k \neq n / 2$, hence the rotating wave will be a saddle. A sufficient condition for these imaginary parts to vanish is that $d_{j}(\varphi)-d_{n-j}(\varphi)$ is even for $1 \leq j \leq m$. It is easy to see that these conditions are satisfied in the case of SCD coupling, i.e. if (5) holds. In this case the persistence condition simplifies to $\overline{\dot{z} p}=0$, and we can expect stable rotating wave oscillations provided that $\overline{\left(\dot{z} f_{\vartheta}\right)^{\prime}}<0$, $\operatorname{Re}\left(l^{(k)}\right)<0$, and the $l_{21}^{(k)}$ have the same signs. Passage through isochronicity then leads to a transition to instability as for the uniform oscillation. In the case of the rotating wave, since generically at least one of the $l^{(k)}$ has a non-zero imaginary part, this transition is usually a torus bifurcation; see Section 3.

\subsection{Cluster oscillations; symmetry $\mathbf{S}_{m} \times \mathrm{S}_{n-m}$}

Now we study the persistence and stability of solutions for which the phases of the oscillators are grouped into two clusters such that the solutions have symmetry $\mathbf{S}_{m} \times \mathbf{S}_{n-m}$. As shown in [3], there is generically at least one non-trivial periodic solution of this form for globally coupled phase oscillators. In fact there can only fail to be such a solution if the in-phase solution is at a bifurcation point. As also shown in [3], there are generically periodic solutions of the 'rotating two-block' type with symmetry $\left(\mathbf{S}_{m} \times \mathbf{S}_{l}\right)^{k} \times{ }_{s} \mathbf{Z}_{k}$ for any decomposition of $n$ into $(m+l) k=n$. However, we do 
not consider these other than in the case $k=1$ in this section and the case $k=2, m=n / 2, l=0$ for the out-of-phase clusters.

The cluster oscillations we study are oscillating solutions of our basic system (1) in a subspace in which $m$ oscillators $\left(v_{i}, u_{i}\right), i \in\left\{i_{1}, \ldots, i_{m}\right\}$, are identical and the remaining $n-m$ oscillators $\left(v_{j}, u_{j}\right)$, $j \in\left\{j_{1}, \ldots, j_{n-m}\right\}$, are identical. Such a subspace is invariant by virtue of the $\mathbf{S}_{n}$-symmetry, and the time evolution in this space is governed by

$$
\begin{aligned}
\frac{d^{2} v_{i}}{d t^{2}}+U^{\prime}\left(v_{i}\right) & =\varepsilon F_{i}\left(v_{i}, u_{i}, v_{j}, u_{j}\right)+O\left(\varepsilon^{2}\right), \\
\frac{d^{2} v_{j}}{d t^{2}}+U^{\prime}\left(v_{j}\right) & =\varepsilon F_{j}\left(v_{j}, u_{j}, v_{i}, u_{i}\right)+O\left(\varepsilon^{2}\right),
\end{aligned}
$$

where

$$
\begin{aligned}
& F_{i}=p\left(v_{i}, u_{i}\right)+(m-1) q\left(v_{i}, u_{i}, v_{i}, u_{i}\right)+(n-m) q\left(v_{i}, u_{i}, v_{j}, u_{j}\right), \\
& F_{j}=p\left(v_{j}, u_{j}\right)+(n-m-1) q\left(v_{j}, u_{j}, v_{j}, u_{j}\right)+m q\left(v_{j}, u_{j}, v_{i}, u_{i}\right) .
\end{aligned}
$$

The corresponding phase-angle system is given by $(k=i, j)$

$$
\begin{aligned}
\frac{d \psi_{k}}{d t} & =\omega_{k}-\varepsilon \omega_{k} z_{k}^{\prime} F_{k}+O\left(\varepsilon^{2}\right), \\
\frac{d \alpha_{k}}{d t} & =\varepsilon \omega_{k} \dot{z}_{k} F_{k}+O\left(\varepsilon^{2}\right),
\end{aligned}
$$

with $\omega_{k}=\omega\left(\alpha_{k}\right), z_{k}=z\left(\psi_{k}, \alpha_{k}\right), v_{k}=z_{k}$ and $u_{k}=\omega_{k} \dot{z}_{k}$.

\subsubsection{Persistence of cluster oscillations}

We set $i=1, j=2$, and compute by means of the Poincaré-Lindstedt method a solution of (21) of the form $(\nu=1,2)$

$$
\begin{aligned}
& \psi_{\nu}(t, \varepsilon)=\varphi+(-1)^{\nu} \Delta / 2+\varepsilon\left[\phi_{\nu}^{(1)}\left(\varphi+(-1)^{\nu} \Delta / 2\right)+\Delta_{\nu}^{(1)}\right]+O\left(\varepsilon^{2}\right), \\
& \alpha_{\nu}(t, \varepsilon)=\alpha^{(0)}+\varepsilon\left[\alpha_{\nu}^{(1)}+\beta_{\nu}^{(1)}\left(\varphi+(-1)^{\nu} \Delta / 2\right)\right]+O\left(\varepsilon^{2}\right),
\end{aligned}
$$

where

$$
d \varphi / d t=\Omega(\varepsilon)=\omega\left(\alpha^{(0)}\right)+\varepsilon \Omega^{(1)}+O\left(\varepsilon^{2}\right)
$$

as in Section 2.1.1. The values of $\alpha^{(0)}$ and the phase shift $\Delta$ have to be determined from the persistence condition (25) below. In the following we set $m_{1}=m, m_{2}=n-m$ and, assuming $\alpha^{(0)}$ and $\Delta$ are known,

$$
\begin{aligned}
& z=z\left(\varphi, \alpha^{(0)}\right), \quad v=z, \quad u=\omega \dot{z}, \\
& z_{\nu}=z\left(\varphi+(-1)^{\nu} \Delta, \alpha^{(0)}\right), \quad v_{\nu}=z_{\nu}, \quad u_{\nu}=\omega \dot{z}_{\nu} \quad(\nu=1,2) \text {. }
\end{aligned}
$$

We now substitute the ansatz (22) into the phase angle system (21). By equating terms at order $O(\varepsilon)$ in $d \alpha_{i} / d t$ we obtain (note that here $\beta_{\nu}^{(1)}=\beta_{\nu}^{(1)}(\varphi)$ )

$$
\dot{\beta}_{\nu}^{(1)}=\dot{z} f_{\nu},
$$

where,

$$
f_{\nu} \equiv p(v, u)+\left(m_{\nu}-1\right) q(v, u, v, u)+m_{\mu} q\left(v, u, v_{\mu}, u_{\mu}\right) \quad(\mu \neq \nu) .
$$

The condition for $\beta_{\nu}^{(1)}$ to be periodic is that the average of the right hand side of (23) vanishes. This leads to the persistence conditions for cluster oscillations,

$$
\overline{\dot{z} f_{\nu}}=0,
$$


two nonlinear equations for $\Delta$ and $\alpha^{(0)}$. Subtracting these equations yields an equation involving the coupling function alone,

$$
\left(m_{1}-m_{2}\right) \overline{\dot{z} q}+m_{2} \overline{\dot{z} q_{2}}-m_{1} \overline{\dot{z} q_{1}}=0,
$$

where we set $q \equiv q(v, u, v, u)$ and $q_{\nu} \equiv q\left(v, u, v_{\nu}, u_{\nu}\right)$.

We assume that $(25)$ has a non-degenerate solution $\left(\alpha^{(0)}, \Delta\right)$, and evaluate in the following all quantities at this solution. At order $O(\varepsilon)$ in $d \psi_{i} / d t$ we then find

$$
\Omega^{(1)}+\omega \dot{\phi}_{\nu}^{(1)}=\omega^{\prime}\left(\alpha_{\nu}^{(1)}+\beta_{\nu}^{(1)}\right)-\omega z^{\prime} f_{\nu}
$$

and the solvability condition for $\phi_{\nu}^{(1)}$ yields

$$
\omega^{\prime} \alpha_{\nu}^{(1)}=\Omega^{(1)}+\omega \overline{z^{\prime} f_{\nu}}
$$

If (27) is satisfied, $\phi_{\nu}^{(1)}$ is obtained by integration of

$$
\dot{\phi}_{\nu}^{(1)}=\overline{z^{\prime} f_{\nu}}-z^{\prime} f_{\nu}+\left(\omega^{\prime} / \omega\right) \beta_{\nu}^{(1)} .
$$

The first order frequency and phase shift corrections are computed in Appendix A.2.1. To simplify notation we introduce the following $2 \pi$-periodic functions of $\varphi$,

$$
\begin{aligned}
q_{, v} & =\left.\frac{\partial q\left(v_{i}, u_{i}, v_{j}, u_{j}\right)}{\partial v_{j}}\right|_{(v, u, v, u)} & q_{, v_{\nu}} & =\left.\frac{\partial q\left(v_{i}, u_{i}, v_{j}, u_{j}\right)}{\partial v_{j}}\right|_{\left(v, u, v_{\nu}, u_{\nu}\right)} \\
q_{, u} & =\left.\frac{\partial q\left(v_{i}, u_{i}, v_{j}, u_{j}\right)}{\partial u_{j}}\right|_{(v, u, v, u)} & q_{, u_{\nu}} & =\left.\frac{\partial q\left(v_{i}, u_{i}, v_{j}, u_{j}\right)}{\partial u_{j}}\right|_{\left(v, u, v_{\nu}, u_{\nu}\right)} \\
d & =q_{, v} \dot{z}+q_{, u} \omega \ddot{z} & d_{\nu} & =q_{, v_{\mu}} \dot{z}_{\mu}+q_{, u_{\mu}} \omega \ddot{z}_{\mu} \\
e & =q_{, v} z^{\prime}+q_{, u}(\omega \dot{z})^{\prime} & e_{\nu} & =q_{, v_{\mu}} z_{\mu}^{\prime}+q_{, u_{\mu}}\left(\omega \dot{z}_{\mu}\right)^{\prime},
\end{aligned}
$$

where $\mu \neq \nu$ and all functions are evaluated at $\alpha^{(0)}$.

\subsubsection{Stability of cluster oscillations}

The eigenvalues of the monodromy matrix for cluster oscillations are analyzed in Appendix A.2.2, and the matrix entries needed for this analysis are computed in Appendix A.2.3. We briefly summarize the results of these calculations.

There are two pairs of transverse, $\left(m_{\nu}-1\right)$-fold degenerate eigenvalues. These eigenvalues are determined by the stability coefficients $(\mu \neq \nu ; \nu, \mu=1,2)$

$$
\begin{aligned}
l_{21}^{(\nu)} & =-2 \pi m_{\nu} \overline{\dot{z} d}-2 \pi m_{\mu} \overline{\dot{z} d_{\nu}}, \\
l^{(\nu)} & =2 \pi \overline{\left(\dot{z} f_{\nu}\right)^{\prime}}-T m_{\nu} \overline{q, u}+2 \pi m_{\mu}\left(\overline{z^{\prime} d_{\nu}}-\overline{\dot{z} e_{\nu}}\right)
\end{aligned}
$$

in the same way as the transverse eigenvalues for the uniform oscillation. Observe that the first term in $l_{21}^{(\nu)}$ vanishes in the case of coupling in the derivatives only, but the second term generically does not. Thus the degeneracy encountered for uniform oscillations in this special case does not occur for cluster oscillations.

The remaining three non-trivial eigenvalues are those of a $4 \times 4$-matrix that governs the stability of the cluster oscillations in their invariant subspace. There is one real eigenvalue $\lambda^{(0)}=1+r \varepsilon+$ $O\left(\varepsilon^{2}\right)$, and a pair of eigenvalues determined by stability coefficients $\left(k_{21}, k\right)$ in the same way as the transverse eigenvalues for the uniform oscillation. The analytical expressions for $\left(r, k_{21}, k\right)$ are presented in equation (A.5). 


\subsection{Other cluster oscillations}

\subsubsection{Out of phase cluster oscillations; symmetry $\left(\mathbf{S}_{n / 2}\right)^{2} \times{ }_{s} \mathbf{Z}_{2}$}

In the case that $n$ is even, $n=2 m$, the results of the preceding subsection apply to out of phase clusters where

$$
m_{1}=m_{2}=m, \quad \Delta=\pi .
$$

If (30) holds, all $2 \pi$-periodic functions with labels $\nu=1$ and $\nu=2$ defined in the last paragraph, in particular those defined in (28), coincide. Thus we can replace these labels by a single label which we set equal to $\pi$, i.e., $z_{1}=z_{2}=z_{\pi}$ with $z_{\pi}(\varphi, \alpha)=z(\varphi+\pi, \alpha)$. Analogously we define $v_{\pi}, u_{\pi}, f_{\pi}$, $d_{\pi}$, and $e_{\pi}$, and note that

$$
f_{\pi}=p(v, u)+(m-1) q(v, u, v, u)+m q\left(v, u, v_{\pi}, u_{\pi}\right) .
$$

The two equations in (25) are now identical and we are left with a single persistence condition,

$$
\overline{\dot{z} f_{\pi}}=0 \text {. }
$$

The eigenvalues of the monodromy matrix are characterized by $\left(l_{21}, l\right)$ (transverse eigenvalues) and $\left(r, k_{21}, k\right)$ (eigenvalues in the space of cluster oscillations) as in the general case. The expressions (A.5) for these stability coefficients simplify to

$$
\begin{aligned}
l_{21} & =-n \pi\left(\overline{\dot{z} d}+\overline{\dot{z} d_{\pi}}\right), \\
l & =2 \pi \overline{\left(\dot{z} f_{\pi}\right)^{\prime}}-\frac{n}{2} T \overline{q_{, u}}+\pi n\left(\overline{z^{\prime} d_{\pi}}-\overline{\dot{z} e_{\pi}}\right), \\
r & =2 \pi \overline{\left(\dot{z} f_{\pi}\right)^{\prime}}, \\
k_{21} & =-2 \pi n \overline{\dot{z} d_{\pi}}, \\
k & =2 \pi \overline{\left(\dot{z} f_{\pi}\right)^{\prime}}+2 \pi n\left(\overline{z^{\prime} d_{\pi}}-\overline{\dot{z} e_{\pi}}\right) .
\end{aligned}
$$

\subsubsection{Degenerate out of phase cluster oscillations for symmetric coupling in the derivatives (SCD)}

If the coupling function $q$ satisfies SCD, i.e. equation (5), then there exist out of phase cluster oscillations $(\Delta=\pi)$ for any arbitrary integers $\left(m_{1}, m_{2}\right)$ with $m_{1}+m_{2}=n$, even for the case $m_{1} \neq m_{2}$. To see this, consider the equation (26). For $\Delta=\pi$ we have $q_{1}=q_{2}=q_{\pi}$, and if (5) holds, $q$ and $q_{\pi}$ are both even. Thus (26) is satisfied and we are left with the single persistence condition $\overline{\dot{z} p}=0$, which is the same as for the uniform oscillation.

The stability coefficients for these out of phase cluster oscillations follow again from the Appendix. Since $e, e_{\pi}$ are now even and $d, d_{\pi}$ are odd, the expressions (A.5) simplify to

$$
\begin{aligned}
l_{21}^{(\nu)} & =-2 \pi\left(m_{\nu} \overline{\dot{z} d}+m_{\mu} \overline{\dot{z} d_{\pi}}\right), \quad \nu=1,2, \\
k_{21} & =-2 \pi n \overline{\dot{z} d_{\pi}} \\
r=k=l^{(\nu)} & =2 \pi \overline{\left(\dot{z} f_{\pi}\right)^{\prime}} .
\end{aligned}
$$

\subsubsection{Cluster oscillations with energy difference of order one}

The cluster oscillations studied so far have energy differences of order $O(\varepsilon)$ between the oscillators in the two clusters. If $\omega(\alpha)$ is monotone, these are the only cluster oscillations we can determine via averaging in the limit $\varepsilon \rightarrow 0$, because the equation $\omega(\alpha)=\omega(\beta)$ admits only the solution $\beta=\alpha$. In 
contrast, if $\omega(\alpha)$ has a local minimum or maximum, i.e. a point of isochronicity, then there exists a solution branch $\beta=h(\alpha)$ to the equation $\omega(\alpha)=\omega(\beta)$ in a certain $\alpha$-range, for which $\beta \neq \alpha$ if $\alpha$ is not an isochronicity value. In this case we can also determine cluster oscillations of the form of $(22)$, but with $\alpha^{(0)}$ for $\nu=2$ replaced by $\beta^{(0)}=h\left(\alpha^{(0)}\right)$, by the method of averaging.

The persistence equations for these cluster oscillations are

$$
\overline{\dot{z} f}=0, \quad \overline{\dot{y} g}=0,
$$

where

$$
\begin{aligned}
f & =p(z, \omega \dot{z})+\left(m_{1}-1\right) q(z, \omega \dot{z}, z, \omega \dot{z})+m_{2} q\left(z, \omega \dot{z}, y_{+}, \omega \dot{y}_{+}\right) \\
g & =p(y, \omega \dot{y})+\left(m_{2}-1\right) q(y, \omega \dot{y}, y, \omega \dot{y})+m_{1} q\left(y, \omega \dot{y}, z_{-}, \omega \dot{z}_{-}\right)
\end{aligned}
$$

and

$$
z=z\left(\varphi, \alpha^{(0)}\right), \quad z_{-}=z\left(\varphi-\Delta, \alpha^{(0)}\right), \quad y=z\left(\varphi, h\left(\alpha^{(0)}\right)\right), \quad y_{+}=z\left(\varphi+\Delta, h\left(\alpha^{(0)}\right)\right), \quad \omega=\omega\left(\alpha^{(0)}\right) .
$$

In Section 3 we numerically compute solutions of these persistence equations for an example. We do not pursue a stability analysis for this type of cluster oscillations; this can be done similarly as in Appendix A.2.

When the persistence energy $\alpha^{(0)}$ is at isochronicity, the equations (25) and (34) are identical. Thus we can expect bifurcations of the two types of cluster oscillations at isochronicity. Moreover, at isochronicity the two equations in (34) coincide for $\Delta=0$, hence we can expect cluster oscillations to bifurcate from the uniform oscillation. Similar considerations apply to the out of phase clusters as well as to higher clusters or clusters with rotating wave components. In summary, isochronicity acts as a degenerate "organizing center" for a variety of oscillation patterns in the averaging framework. We do not attempt a detailed analysis of the bifurcations organized by isochronicity in this paper. Some examples are given in Section 3, where we also elucidate special features of the bifurcation structure due to SCD coupling.

\section{Example: a system of weakly coupled dissipative oscillators with quartic potential}

We consider oscillators (1) with a quartic potential

$$
U(v)=v^{4} / 4-v^{2}-2 v .
$$

This potential is a special case of the one parameter family of potential normal forms $U(v, r)=$ $v^{4} / 4-v^{2}-r v$ studied by Chow and Sanders [9], who show that for $r>\sqrt{32 / 27}$ there exists a point of isochronicity. After a brief discussion of the Hamiltonian system, we will study the associated system of four globally coupled oscillators with a van der Pol type dissipation function and coupling that is cubic in the positions and linear in the derivatives:

$$
\frac{d^{2} v_{i}}{d t^{2}}+U^{\prime}\left(v_{i}\right)=-\varepsilon\left(\left(v_{i}-2\right)^{2}-s\right) u_{i}+\varepsilon \sum_{j=1}^{4}\left(c_{1}\left(v_{j}-v_{i}\right)+c_{2}\left(u_{j}-u_{i}\right)+c_{3}\left(v_{j}-v_{i}\right)^{3}\right) .
$$

The system (36) has special cases of coupling as described in Section 1.1, namely CDO if $c_{1}=c_{3}=0$ and CPO (i.e. SCD) if $c_{2}=0$. We perform averaging calculations on fixing the coupling coefficients and treating $s$ as bifurcation parameter. Complementary to this we perform simulations and apply numerical continuation methods to find bifurcation diagrams for the full system for fixed values of

$s$ and varying coupling constants. Simulations and continuation of periodic solutions were carried out using the package XPPAUT [12]. 


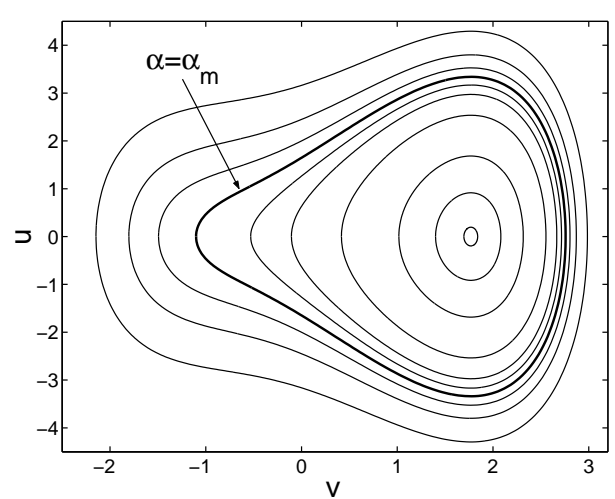

(a)

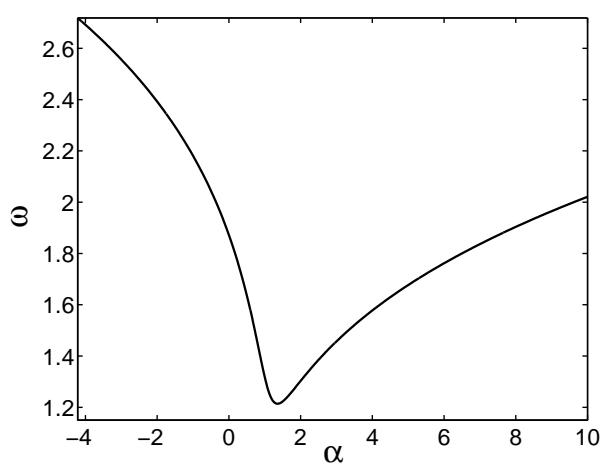

(b)

Figure 1: Hamiltonian oscillator for the potential (35). (a): Level sets $u^{2} / 2+U(v)=\alpha$. (b): Frequency-energy function $\omega(\alpha)$.

\subsection{Averaging for quartic oscillators}

\subsubsection{Single oscillator}

Some level sets $H=\alpha$ of the Hamiltonian function $H(v, u)=u^{2} / 2+U(v)$ are displayed in Figure 1 (a). The minimum of the potential $U(v)$, equation (35), is $\alpha_{\min }=-4.2191$ and occurs at $v_{\min }=1.7693$. Denote by $-a \pm i b$ the complex roots of the quartic polynomial $\alpha-U(v)$ for $\alpha>\alpha_{\text {min }}$. The real part $-a$ and $\alpha$ are related by

$$
\alpha=1 / a^{2}-\left(a^{2}-1\right)^{2} .
$$

When $\alpha$ increases from $\alpha_{\min }$ towards infinity, $a$ decreases monotonically from $v_{\min }$ towards zero. We therefore can use $a$ as parameter instead of $\alpha$. In terms of $a$, the real roots of $\alpha-U(v)$ are $a \pm \sqrt{2 / a+2-a^{2}}$, and the imaginary part of the complex root is $b^{2}=a^{2}+2 / a-2$.

The period function can be written in terms of Jacobi's complete elliptic integral $K(k)$ as

$$
T(a)=4 \sqrt{\frac{a}{D_{1}}} K(k)
$$

with the modulus

$$
k=\frac{D}{\sqrt{2 D_{1}\left(D_{1}+3 a^{3}-2 a\right)}}
$$

and where

$$
D=\sqrt{4-a^{2}\left(a^{2}-2\right)^{2}}, \quad D_{1}=2 \sqrt{2 a^{6}-2 a^{4}+1} .
$$

In Figure 1 (b) we show the frequency $\omega=2 \pi / T$ as function of $\alpha$. Isochronicity occurs at $\alpha_{m}=$ 1.3577 and corresponds to a frequency minimum, $\omega\left(\alpha_{m}\right)=1.2139$. The associated value of $a$ is $a_{m}=0.8288$.

The $2 \pi$-periodic solution $z(\varphi, a)$ of the equation

$$
\omega^{2}(a) \ddot{z}+U^{\prime}(z)=0,
$$

can be represented in terms of Jacobi's elliptic function $\mathrm{cn}$ in the form

$$
z(\varphi, a)=\frac{r_{1} \operatorname{cn}(\tau, k)+r_{2}}{r_{3} \operatorname{cn}(\tau, k)+r_{4}}
$$


where $\tau=\sqrt{D_{1} / a}(\varphi / \omega)$,

$$
\begin{array}{ll}
r_{3}=2 a+d-b / k_{1}, & r_{1}=a r_{3}-d r_{4}, \\
r_{4}=2 a+d+b k_{1}, & r_{2}=a r_{4}-d r_{3},
\end{array}
$$

and

$$
k_{1}=\left(3 a^{3}-2 a+D_{1}\right) / D, \quad d=\sqrt{2 / a+2-a^{2}} .
$$

In our averaging calculations we work with the analytical representations of $z$ and $\dot{z}$. The energy derivatives $z^{\prime}$ and $\dot{z}^{\prime}$ are computed numerically by solving their governing variational equations. The averages required in the perturbation analysis are computed numerically using the trapezoidal rule.

\subsubsection{Oscillating solutions for the coupled system}

We now give a brief overview of the oscillating solutions studied in the remainder of this section.

Basic solutions As was discussed in Section 1.2, the basic solutions for generic coupling are the uniform oscillation, $U O$, the rotating wave oscillation, $R W$, and the out of phase cluster oscillation, $O P C$. For SCD coupling $\left(c_{2}=0\right)$ we also find $(1,3)$-out of phase cluster oscillations as basic solutions, which we denote here by $O P C 13$.

In Appendix A.3 we summarize the persistence conditions and stability coefficients for these solutions. Due to our special form of coupling, the persistence condition for $U O$ is the same as for a single Hamiltonian oscillator perturbed by the $O(\varepsilon)$-dissipation term, i.e.

$$
\overline{\dot{z}^{2}(z-2)^{2}}-s \overline{\dot{z}^{2}}=0 .
$$

In Figure 2 we show the persistence energy $\alpha(s)$ determined by equation (37). We see that $\alpha(s)$ increases monotonically with $s$ and passes through isochronicity at $s_{c}=1.5298, \alpha\left(s_{c}\right)=\alpha_{m}$. For $c_{2}=0$, the persistence conditions for all four basic solutions $U O, R W, O P C, O P C 13$ are identical (see Appendix A.3), hence their persistence energies are determined by $\alpha(s)$ as well. If $c_{2} \neq 0, \alpha(s)$ only determines the persistence energy of $U O$.

Inspection of the stability coefficients shows:

- The stability of $U O$ depends only on $c_{1}$ : if $c_{1}>0, U O$ is stable for $s>s_{c}$ and a saddle for $s<s_{c}$, and vice versa for $c_{1}<0$.

- For $R W$ there is a complex conjugate pair $\left(l_{21}^{(1,3)}, l^{(1,3)}\right)$ with $l_{21}^{(1,3)}$ real if $c_{2}=0$, and a real pair $\left(l_{21}^{(2)}, l^{(2)}\right)$. This solution can only be stable if $c_{2}=0$.

- For both $O P C$ and $O P C 13$ there is a pair $\left(l_{21}, l\right)$ of multiplicity two, and a pair $\left(k_{21}, k\right)$ of multiplicity one. If $c_{2}=c_{3}=0, O P C$ and $O P C 13$ are both saddles.

Two-cluster oscillations In the averaging framework we have to distinguish cluster oscillations with energy differences of order $O(\varepsilon)$, denoted $C_{\varepsilon}(2,2)$ and $C_{\varepsilon}(1,3)$, and of order one, denoted $C_{1}(2,2)$ and $C_{1}(1,3)$, respectively. The $1: 1$ resonance set $\omega(\beta)=\omega(\alpha)$ for our Hamiltonian oscillator is shown in Figure 3. For $C_{\varepsilon}(2,2)$ and $C_{\varepsilon}(1,3)$ the leading order energies $(\alpha, \beta)$ of the two clusters are identical, i.e. on the branch $\beta=\alpha$ of the resonance set, and for $C_{1}(2,2)$ and $C_{1}(1,3)$ they are on the branch $\beta=h(\alpha)$. We note that the distinction of the two types of cluster oscillations makes strict sense only in the asymptotic limit $\varepsilon \rightarrow 0$. 


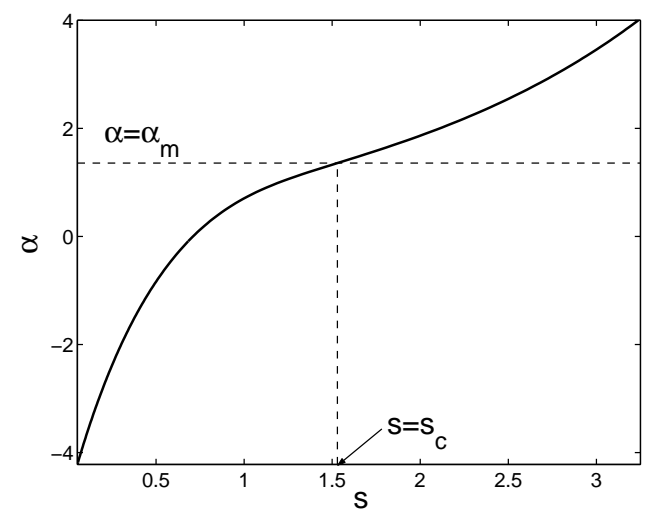

Figure 2: Persistence energy $\alpha(s)$ determined by (37). This persistence energy holds for all four basic solutions ( $U O, R W, O P C, O P C(1)=O P C 13)$ if $c_{2}=0$. If $c_{2} \neq 0, \alpha(s)$ only governs $U O$. Isochronicity occurs at $s_{c}=1.5298$.

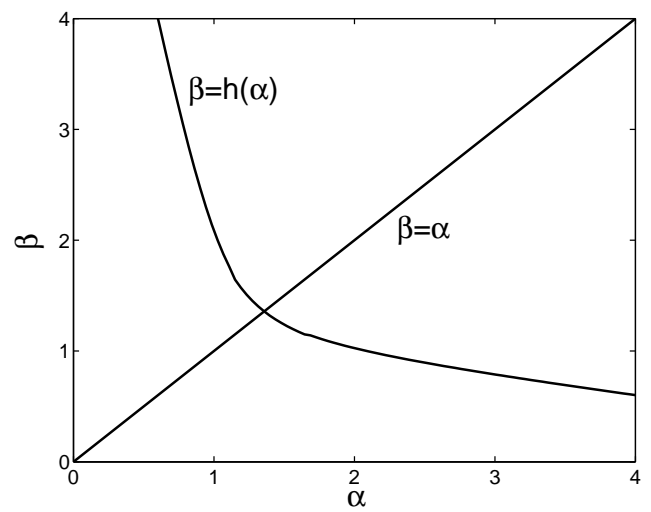

Figure 3: Resonance curves $\omega(\alpha)=\omega(\beta)$ for a Hamiltonian oscillator with potential (35). The branch $\beta=h(\alpha)$ crosses the trivial branch $\beta=\alpha$ at the isochronicity energy $\alpha_{m}=1.3577$.

The transverse stability of $C_{\varepsilon}(2,2)$ and $C_{\varepsilon}(1,3)$ is determined by two pairs $\left(l_{21}^{( \pm)}, l^{( \pm)}\right)$of multiplicity one and a single pair $\left(l_{21}, l\right)$ of multiplicity two, respectively. The stability in the space of two-cluster oscillations for both types is determined by a pair $\left(k_{21}, k\right)$ and a single term $r$, analogously as for the out of phase clusters. Numerically we found that $k, l^{( \pm)}, l, r<0$ for the parameter ranges considered.

In Appendix A.3 we summarize the persistence conditions for all four cluster oscillations, and the stability coefficients $l_{21}, l_{21}^{( \pm)}$and $k_{21}$ for $C_{\varepsilon}(2,2)$ and $C_{\varepsilon}(1,3)$. We have not computed stability coefficients for $C_{1}(2,2)$ and $C_{1}(1,3)$.

Three- and four-cluster oscillations In our simulations we also observe three-cluster oscillations $(2,1,1)$, with the two oscillators in the first cluster identical, and the four-cluster oscillation $(1,1,1,1)$. We do not analyze these oscillations via averaging, but continue them for varying coupling coefficients using AUTO. 


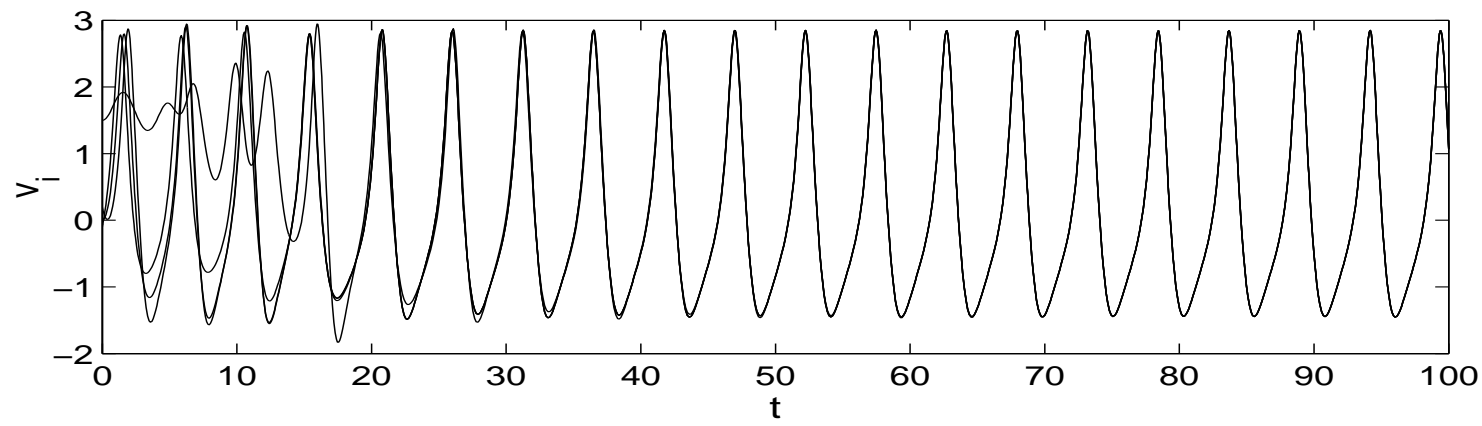

(a)

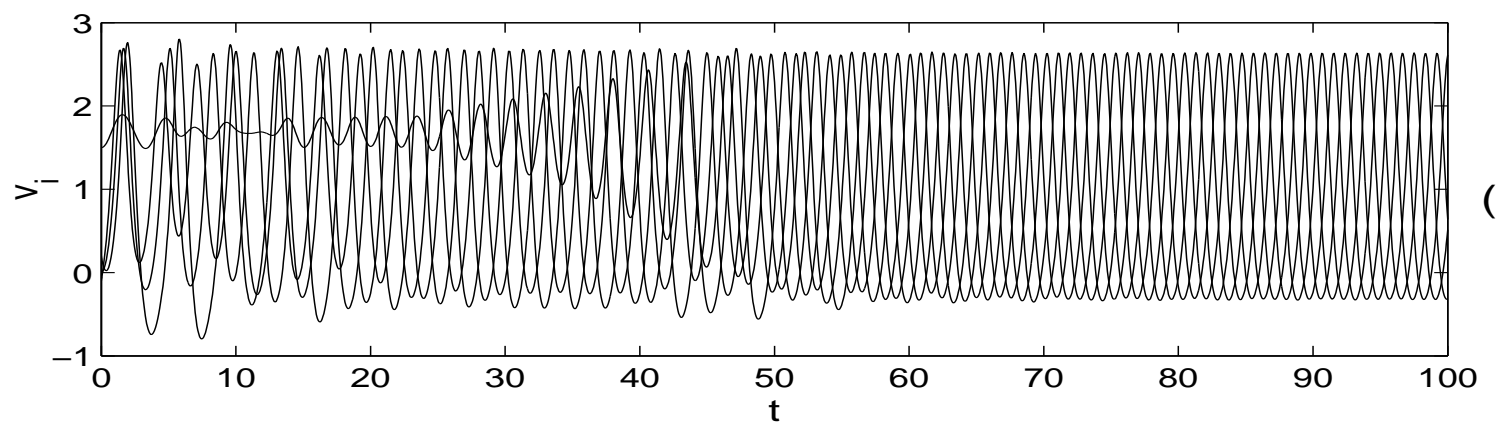

(b)

Figure 4: Time series for (36) with $\varepsilon=0.2, c_{1}=1, c_{2}=c_{3}=0$, and initial conditions $\left(v_{1}, v_{2}, v_{3}, v_{4}\right)=(0.2,-0.1,1.5,0),\left(u_{1}, u_{2}, u_{3}, u_{4}\right)=(-1,1,0,0)$. In (a) for $s=2$ we see approach to $U O\left(s>s_{c}\right)$, and in (b) for $s=1$ we have approach to $R W\left(s<s_{c}\right)$.

\subsection{Linear and cubic coupling in the positions}

For coupling in the positions only, $c_{2}=0$, the persistence energies of the basic solutions $U O, R W$, $O P C$, and $O P C 13$ are identical and vary monotonically with $s$, see Figure 2 (a).

If $c_{3}=0, O P C$ and $O P C 13$ are saddles, and $U O$ and $R W$ exchange stability at isochronicity. This is demonstrated in Figure 4 (a), (b) for $c_{1}=1$ : for $s>s_{c}$ we see approach to $U O$ and for $s<s_{c}$ to $R W$ for the same initial condition.

If $c_{3} \neq 0$, the stability coefficients $l_{21}^{(1,3)}, l_{21}^{(2)}$ for $R W$ and $l_{21}, k_{21}$ for $O P C$ and $O P C 13$ can change their signs. In Figure 5 we show the curves in the $\left(c_{1} / c_{3}, s\right)$-plane where these coefficients vanish. Together with the isochronicity line $s=s_{c}$, these curves define codimension one boundaries dividing the $\left(c_{1} / c_{3}, s\right)$-plane into regions with different stability assignments as shown in the figure. Note that we have omitted the sign of $r$ in the stability assignments because $r$ is always negative.

On $\left\{l_{21}^{(2)}=0\right\}$ we find a transverse bifurcation from $R W$ to a $(2,2)$-cluster with the oscillators in each cluster $\pi$ out of phase. On $\left\{l_{21}^{(1,3)}=0\right\}$ there is a torus bifurcation from $R W$ to a quasiperiodic rotating wave because $l^{(1,3)}$ is complex. On $\left\{l_{21}=0\right\}$ for $O P C$ and $O P C 13$ we find a transverse bifurcation to a $(2,1,1)$-cluster, and on $\left\{k_{21}=0\right\}$ a bifurcation to a generic $(2,2)$-cluster $C_{\varepsilon}(2,2)$ and $C_{\varepsilon}(1,3)$, respectively. Note that the boundaries $\left\{k_{21}=0\right\}$ are identical for $O P C$ and $O P C 13$ in the averaging framework.

The averaging approach admits cluster oscillations of types $C_{\varepsilon}(2,2)$ and $C_{\varepsilon}(1,3)$ only if $c_{1}$ and $c_{3}$ have opposite signs. The phase shifts $\Delta$ versus $s$ for these solutions are shown in Figure 6 for $c_{1}=-1$ and six values of $c_{3}$. These branches are identical in the averaging limit and bifurcate from the (identical) boundaries $\left\{k_{21}=0\right\}$ for $O P C$ and $O P C 13$ shown in Figure 5 (b) and (c). The persistence energies along these branches are simply segments of the graph of Figure 2 (a). For the chosen parameter values both $C_{\varepsilon}(2,2)$ and $C_{\varepsilon}(1,3)$ are saddles and have the same stability 


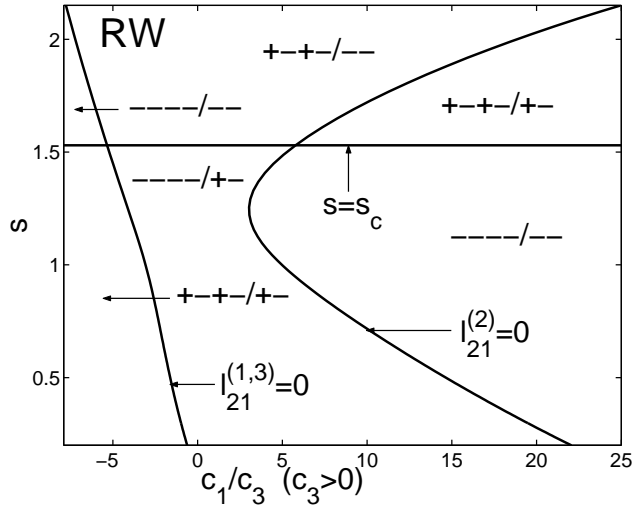

(a)

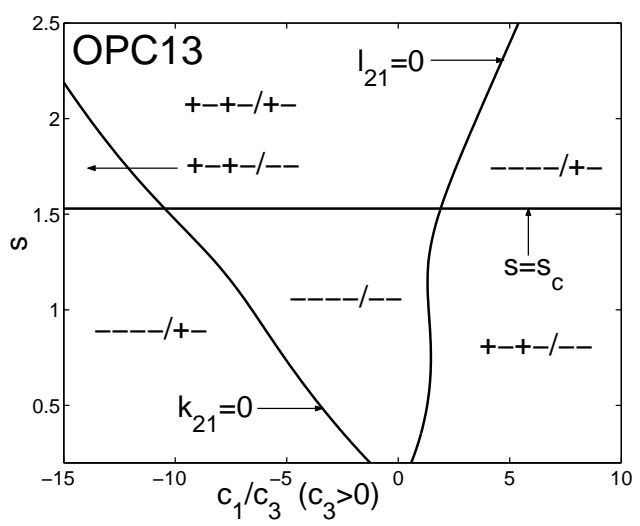

(c)

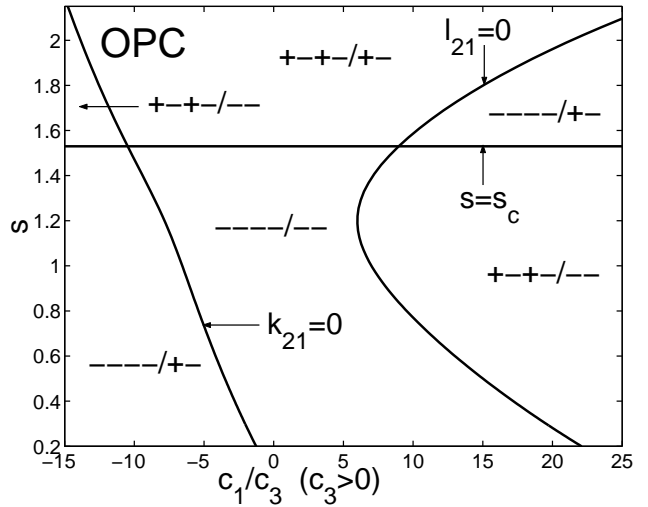

(b)

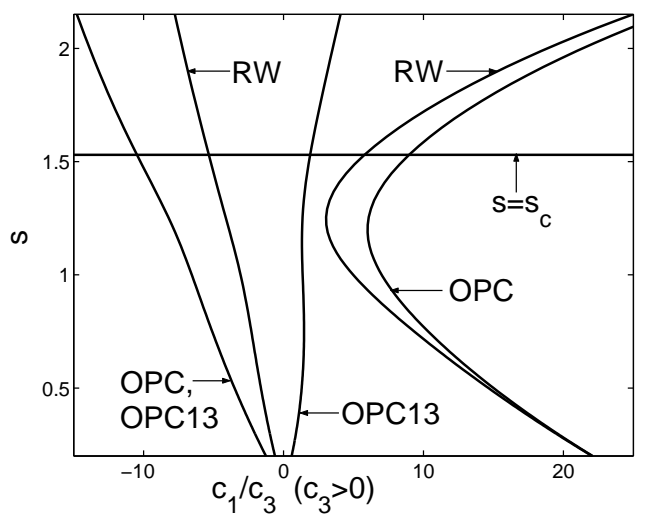

(d)

Figure 5: (a)-(c): Stability diagrams for the basic solutions of (36) with $c_{2}=0$ in the $\left(c_{1} / c_{3}, s\right)-$ plane. (a): RW, (b): OPC, (c): OPC13. (d): Codimension one boundaries of (a)-(c) in one diagram.

assignments. At isochronicity the stability assignments change as marked in the figure.

In Figure 7 (a), (b) and (c), (d) we show bifurcation diagrams for the cluster oscillations $C_{1}(1,3)$ and $C_{1}(2,2)$ for $c_{1}=c_{3}=1$ and $-c_{1}=c_{3}=1$, respectively. The phase shifts are shown in (a), (c), and the energies in (b), (d), as functions of $s$. For $c_{1}=c_{3}=1$, these cluster oscillations bifurcate transcritically from $U O$ and their out of phase versions $O P C$ and $O P C 13$ at isochronicity. For $-c_{1}=c_{3}=1$, there are also cluster oscillations of types $C_{\varepsilon}(1,3)$ and $C_{\varepsilon}(2,2)$. In this case we find at isochronicity an additional transcritical bifurcation of $C_{\varepsilon}(1,3)$ and $C_{1}(1,3)$, and $C_{\varepsilon}(2,2)$ and $C_{1}(2,2)$. This confirms the observation made in Section 2 that isochronicity acts as a degenerate bifurcation point that affects all basic solutions and cluster oscillations in the limit $\varepsilon \rightarrow 0$. We have not studied in detail how these bifurcations "unfold" for $\varepsilon \neq 0$.

Bifurcation diagrams for the full system (36) are shown in Figure 8 for $\varepsilon=0.05, c_{3}=0.1$, and varying $c_{1}$ for (a): $s=1.8$, and (b): $s=1.2$. These bifurcation diagrams correspond to horizontal paths through the stability diagrams of Figure 5 above and below isochronicity. In (a) we see the transverse instability of $U O$ near $c_{1}=0$ and the torus bifurcation of $R W$ on the line $\left\{l_{21}^{(1,3)}=0\right\}$ of Figure 5 (a). The branch 2,2 corresponds to a cluster branch of type $C_{1}(2,2)$. We see that this branch bifurcates supercritically from $U O$ as in Figure 7 (c), (d) and is stable. We also see $(2,1,1)$ and $(1,1,1,1)$ cluster oscillations emanating stably from $U O$. In the limit $\varepsilon \rightarrow 0$, all these symmetry breaking bifurcations merge at $c_{1}=0$. 


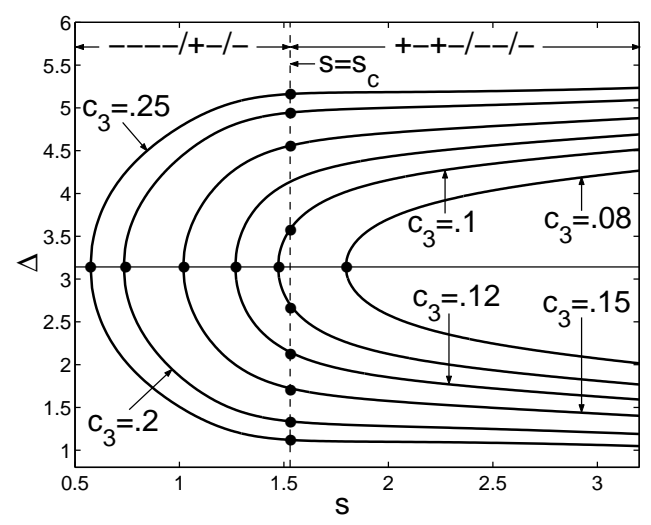

Figure 6: Phase shifts $\Delta$ of $C_{\varepsilon}(1,3)$ and $C_{\varepsilon}(2,2)$ versus $s$ for (36) with $c_{2}=0, c_{1}=-1$, and six values of $c_{3}$. At leading order averaging the phase shifts for these solutions are identical in case of SCD, and the persistence energies coincide with the persistence energy for UO. For the chosen parameter values the stability assignments for the two solutions are the same and change at isochronicity as marked in the figure.

In Figure 8 (b), below isochronicity, we see the instability of $R W$ on the line $\left\{l_{21}^{(2)}=0\right\}$ of Figure 5 (a). Here no stable oscillation bifurcates from $R W$. Instead, complicated period doublings occur near $c_{1}=-0.8$ which have not been investigated in detail. Again we see the supercritical pitchfork of $C_{1}(2,2)$ and this time also a stable $C_{1}(1,3)$ branch. Figure 9 gives some examples of timeseries of differences on the attractors for the same system.

\subsection{Mixed linear coupling}

Now we study the effect of additional coupling in the derivatives, $c_{2} \neq 0$, for the case of linear couplings, $c_{3}=0$. If $c_{2} \neq 0$, the persistence energies of the basic solutions $U O, R W$, and $O P C$ no longer coincide, and each of them has its own isochronicity value. Moreover, $R W$ is always a saddle, and $O P C$ is a saddle as well if the coupling is linear. In Figure 10 we show the persistence energies of the basic solutions for $c_{1}=1$ and four different values of $c_{2}$. Notice that $O P C$ encounters a saddle node bifurcation for larger values of $c_{2}$. The stability of $U O$ is unchanged, i.e. it is stable for $s>s_{c}$ and a saddle for $s<s_{c}$.

Cluster oscillations of type $C_{\varepsilon}(2,2)$ do not occur in the averaging framework if the coupling is linear. In contrast, $O P C 13$ for $c_{2}=0$ evolves into a unique $C_{\varepsilon}(1,3)$ branch for $c_{2} \neq 0$. In Figure 11 we show the phase shifts and energies versus $s$ for these oscillations for $c_{1}=1$ and the four values of $c_{2}$ chosen for Figure 10. Shown is also the isochronicity curve in the phase shift diagram. Note that $C_{\varepsilon}(1,3)$ is always a saddle as is $R W$ and $O P C$.

Cluster oscillations of types $C_{1}(2,2)$ and $C_{1}(1,3)$ both exist and their bifurcation for varying $s$ is displayed in Figure 12 for $c_{1}=c_{2}=1$. As in the case $c_{2}=0$, we see $C_{1}(2,2)$ bifurcating from $U O$ and $O P C$ in pitchforks, whereas $C_{1}(1,3)$ bifurcates from $U O$ and $C_{\varepsilon}(1,3)$ transcritically. We conjecture that the transcritical bifurcation of $C_{\varepsilon}(1,3)$ and $C_{1}(1,3)$ "unfolds" in the usual way in a saddle node pair or in two monotonic branches when $\varepsilon$ becomes nonzero, but this requires further investigation. In contrast, the bifurcation of $C_{1}(2,2)$ from $U O$ and $O P C$ is a true symmetry breaking bifurcation revealed in pitchforks. This time the pitchfork bifurcation of $C_{1}(2,2)$ from $U O$ 

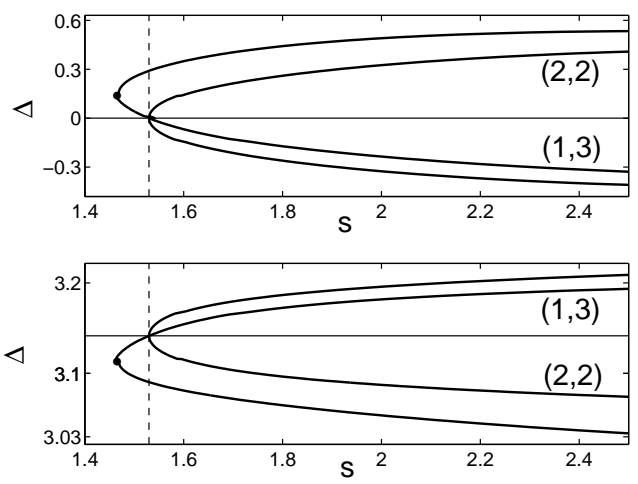

(a)
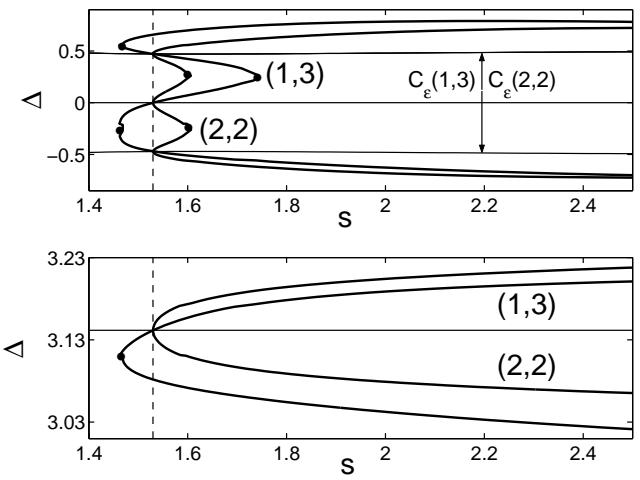

(c)

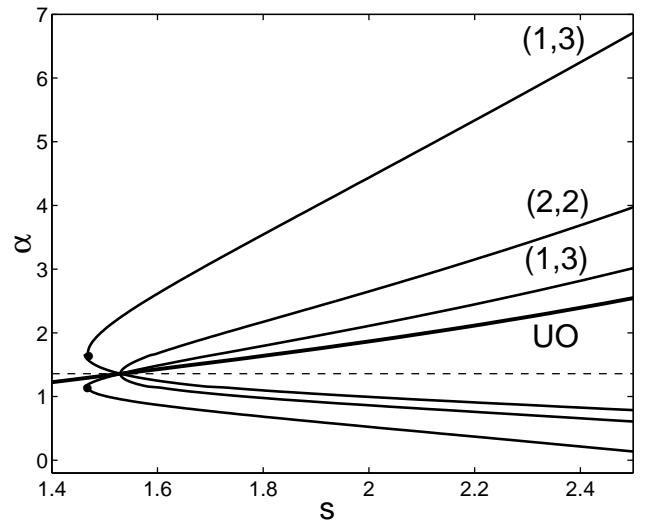

(b)

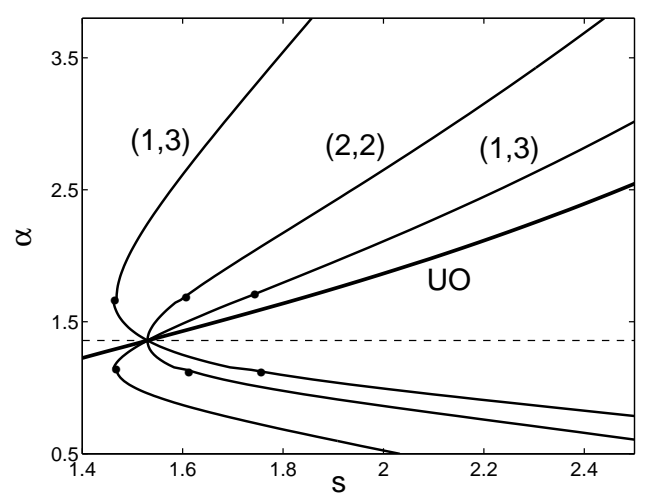

(d)

Figure 7: Bifurcation of $C_{1}(2,2)$ and $C_{1}(1,3)$ for (36) with $c_{2}=0$ and $(a),(b): c_{1}=c_{3}=1$, (c), $(d):-c_{1}=c_{3}=1$. (a), (c): Phase shifts versus $s$. In the top diagram of (c) we also show the phase shifts of $C_{\varepsilon}(2,2)$ and $C_{\varepsilon}(1,3)$, cf. Figure 6 . (b), (d): Both energies of the cluster oscillators and uniform persistence energy versus $s$ with saddle nodes marked by dots. Note that in (a), (b) $U O$ is stable for $s>s_{c}$, and in (c), (d) for $s<s_{c}$. Thus the pitchfork of $C_{1}(2,2)$ from UO is subcritical in (a), (b) and supercritical in (c), (d).

is subcritical. We have not computed the stability assignments of these cluster branches using the averaging approach. Our simulations and AUTO calculations suggest that they are both saddles.

In the case of coupling in the derivatives only, CDO, where $c_{1}=c_{3}=0$ we observe a large range of possible attractors and quite sensitive dependence on initial conditions and parameters. Figure 13 illustrates some simulations for the case with $s=1.8$ and $\varepsilon=0.05$. On reducing the value of $c_{2}$ we go from stable UO to stable RW, then to a variety of intermittent attractors, some of which we believe may be structurally stable heteroclinic cycles. For $c_{2}=-0.5$ we find a state that appears to be fully symmetric chaos; thus we have a very similar scenario for breakdown to fully symmetric chaos to that previously observed in [4]. However, we have not been able to confirm the details even for the structurally stable heteroclinic cycles because several of the Floquet multipliers obtained from continuation are very close to being unity, in agreement with the analytical calculations of Section 2.1.3.

Nonetheless, for example in Figure 14 we show that at larger values of $\varepsilon$ and for $c_{1}, c_{2} \neq 0$ there are clearly structurally stable heteroclinic attractors. 


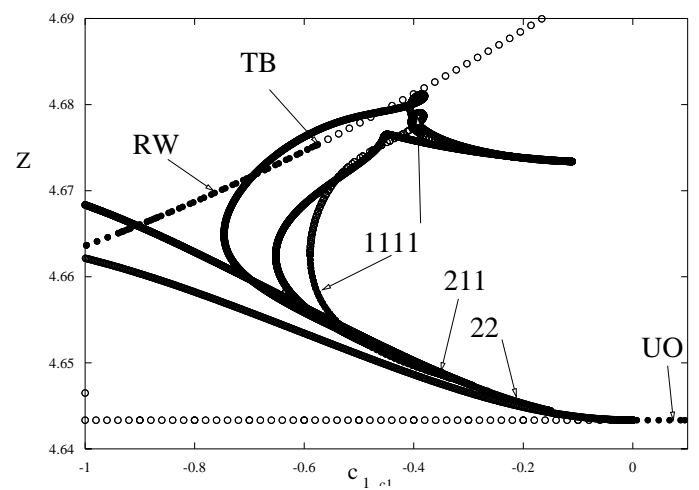

(a)

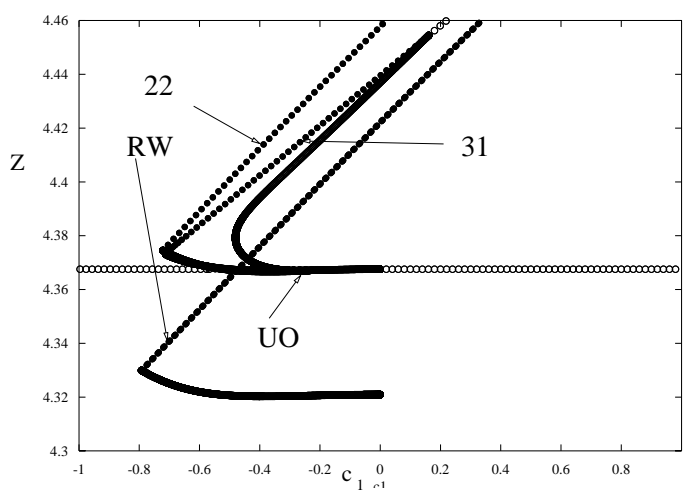

(b)

Figure 8: Bifurcation diagrams showing mean solution norm $Z$ versus $c_{1}$, calculated using AUTO, for (36) with $\varepsilon=0.05, c_{2}=0, c_{3}=0.1$, and (a): $s=1.8,(b): s=1.2$. TB marks a torus bifurcation of $R W$. Branches 2, 2 and 1,3 are branches of two-cluster oscillations with $O(1)$ energy differences. Branches 2,1,1 and 1,1,1,1 are branches of three-and four-cluster oscillations. The filled circles correspond to stable branches.

\section{A phase-energy model for coupled oscillators near isochronicity}

In [6] we derived a reduced, coupled phase energy system for weakly perturbed Hamiltonian systems that describes the dynamics near a point of isochronicity for the case that the coupling terms are of order $O\left(\varepsilon^{2}\right)$, and the dissipation is of order $O(\varepsilon)$. Although this phase energy system is a reduction of the original system (1), even in the limit of small $\varepsilon$ it can be technically very difficult to calculate the reduced functions in terms of integrals of solutions of the Hamiltonian oscillators; often one needs to use partly numerical methods even for the case of quite simple potential $U(v)$.

Therefore, in an attempt to better understand the dynamics of coupled oscillators that depend in addition to phases on energy-type variables and are not necessarily weak perturbations of Hamiltonian systems, we consider the following phase-energy model:

$$
\begin{aligned}
\dot{\theta}_{i} & =\omega\left(\alpha_{i}\right)+\frac{\varepsilon}{n} \sum_{j=1}^{n} f\left(\theta_{j}-\theta_{i}\right), \\
\dot{\alpha}_{i} & =-\beta\left(\alpha_{i}-\alpha_{0}\right)+\frac{\varepsilon}{n} \sum_{j=1}^{n} g\left(\theta_{j}-\theta_{i}\right) .
\end{aligned}
$$

The phase of oscillator $i$ is represented by the angular variable $\theta_{i}$ (modulo $2 \pi$ ) and the energy relative to the uncoupled $(\varepsilon=0)$ level is represented by $\alpha_{i} \in \mathbf{R}$. We set

$$
\omega(\alpha)=1+\lambda_{1} \alpha+\lambda_{2} \alpha^{2} .
$$

This is inspired by the system (6), but $\beta>0$ need not be small. We say such oscillators have isochronicity at energy $\alpha$ if $\omega^{\prime}(\alpha)=0$; for example they are isochronous at 0 if and only if $\lambda_{1}=0$. The system (38) has a continuous phase-shift symmetry given by

$$
\theta_{i} \mapsto \theta_{i}+\varphi
$$

for all $i$ introduced by averaging, and this allows one to easily locate periodic orbits of this equation. The phase shift symmetry can be removed by considering more general arbitrary $f\left(\theta_{j}, \theta_{i}\right)$ and 
(a)

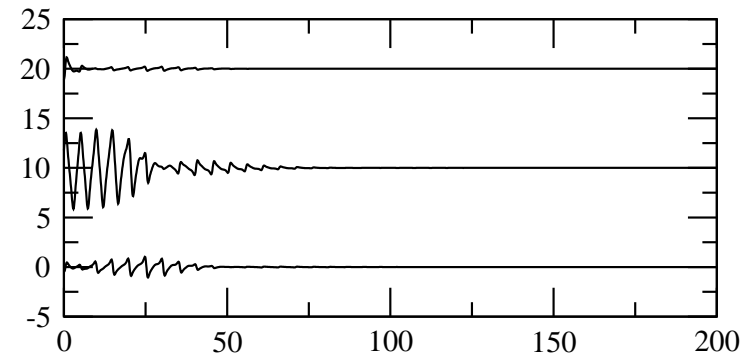

(c)

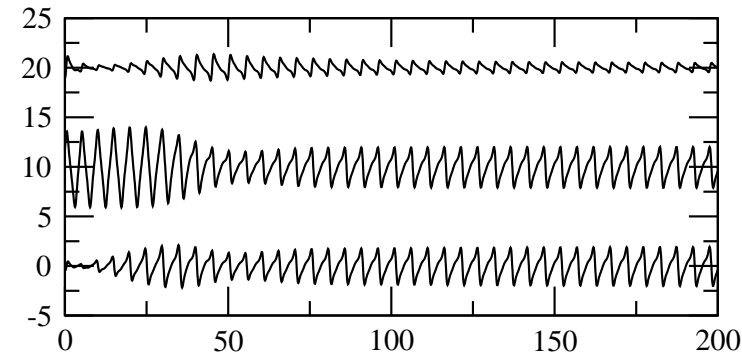

(e)

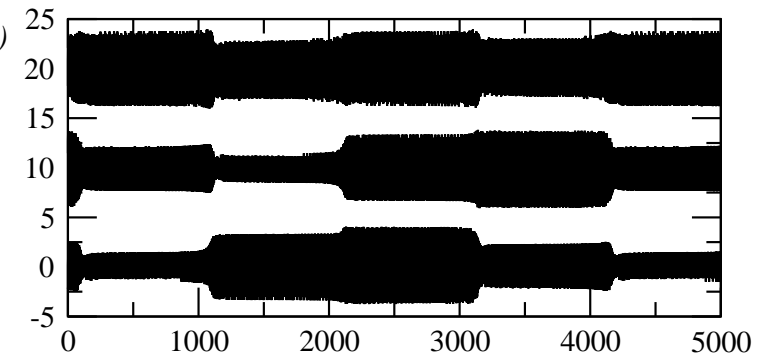

(b)

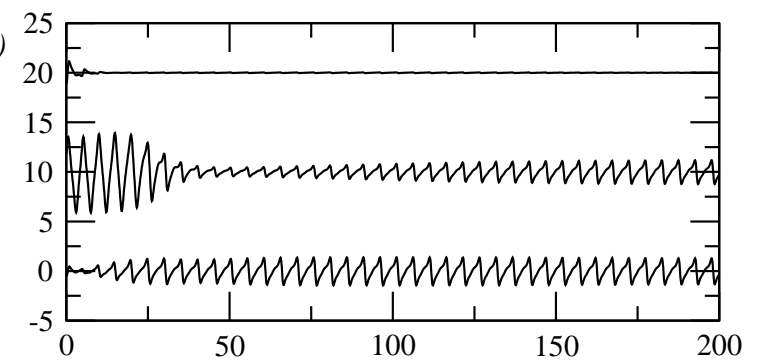

(d)
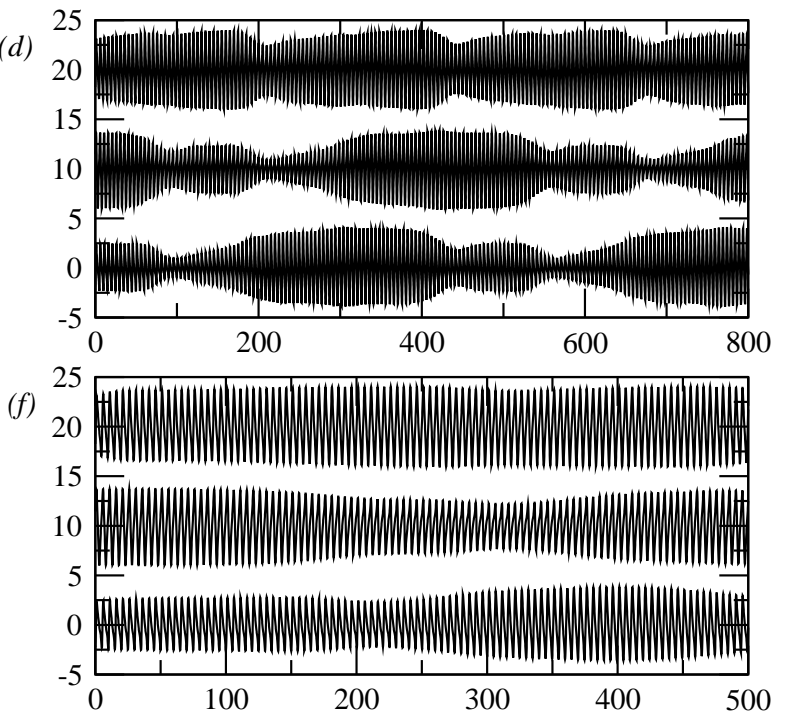

Figure 9: Simulations for the four coupled oscillators with CPO, $c_{2}=0, c_{3}=0.1, \varepsilon=0.05$ and $s=1.8$. For $(a)-(f)$ we have set $c_{1}=0.1,-0.1,-0.3,-0.35,-0.387,-0.5$ respectively. In all plots the differences $v_{1}-v_{k}+10(k-2)$ for $k=2,3,4$ against time (horizontal axis) are shown; a flat section of the plot indicates that two oscillators are synchronized during this time. Observe that (a) shows a transient to attracting UO, (b) a $(2,2)$ cluster, (c) a $(2,1,1)$ cluster, (d) is close to a heteroclinic orbit, while (e) is a torus that has bifurcated from a $R W$.

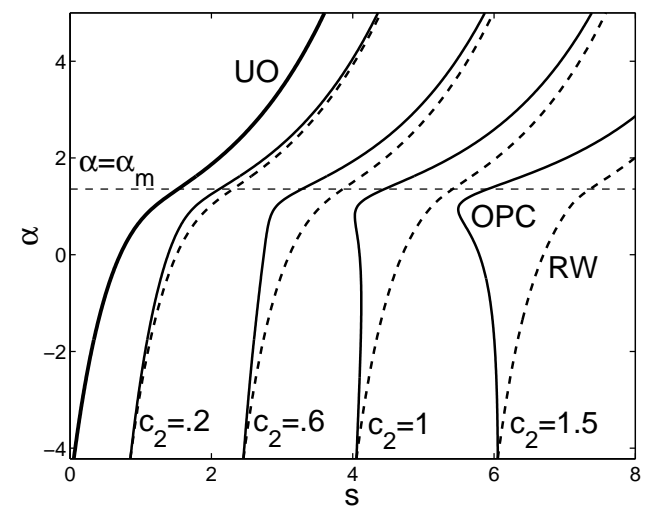

Figure 10: Persistence energies $\alpha$ versus $s$ for (36) with $c_{3}=0, c_{1}=1$, and four different values of $c_{2}$. Thick line: UO, full lines: OPC, dashed lines: $R W$. 


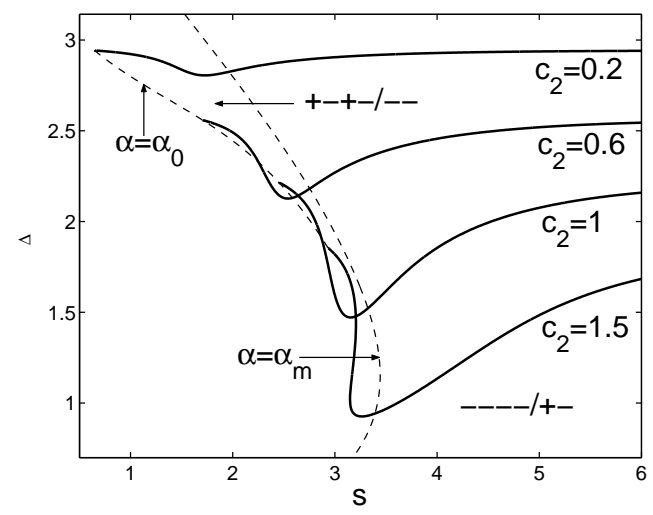

(a)

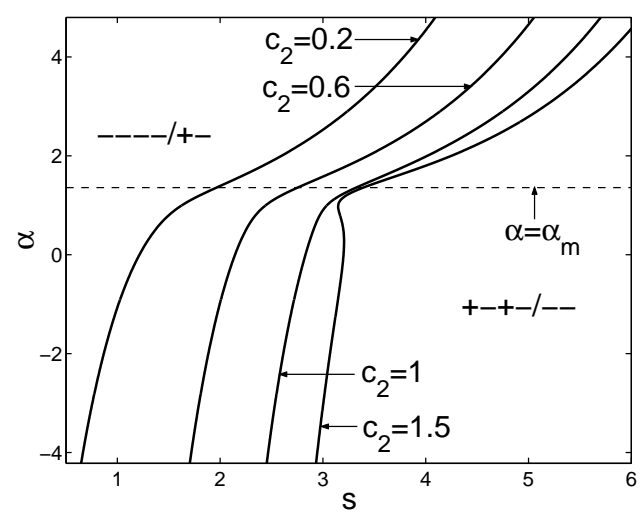

(b)

Figure 11: Cluster oscillation $C_{\varepsilon}(1,3)$ for (36) with $c_{1}=1, c_{3}=0$, and four different values of $c_{2}$. (a): $\Delta$ versus $s,(b): \alpha$ versus $s$. The left dashed curve in (a) marks the location where the branches emanate from the energy minimum. On the right dashed curve the branches pass through isochronicity.

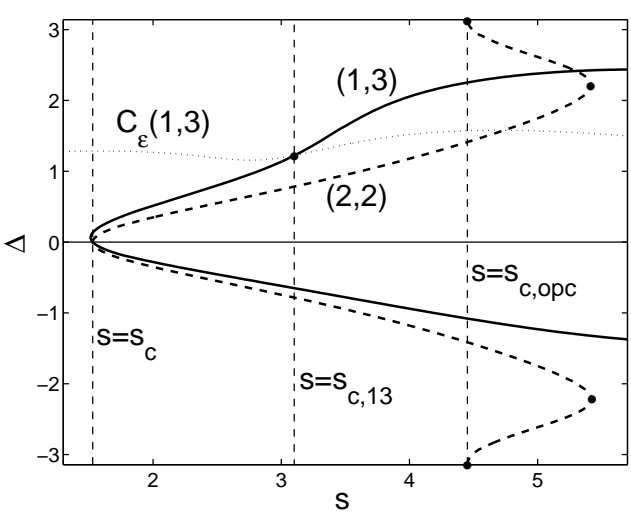

(a)

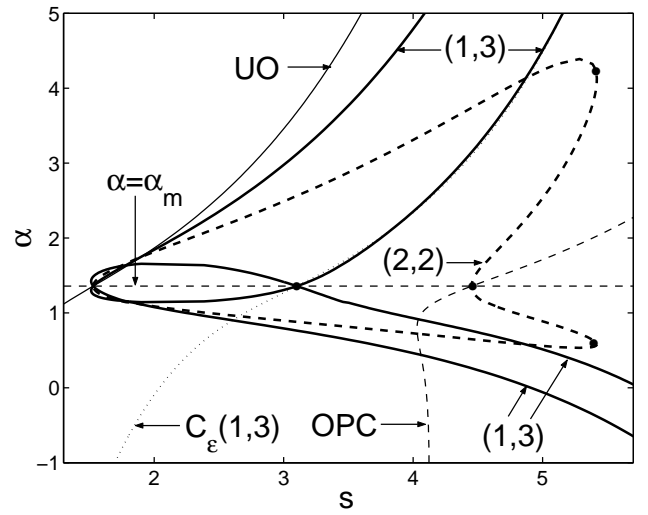

(b)

Figure 12: Bifurcation of $C_{1}(1,3)$ and $C_{1}(2,2)$ for (36) with $c_{1}=c_{2}=1, c_{3}=0$. (a): Phase shifts versus s. Also shown is the phase shift of $C_{\varepsilon}(1,3)$. (b): Both energies of $C_{1}(1,3)$ and $C_{1}(2,2)$, and the energies of $U O, O P C$, and $C_{\varepsilon}(1,3)$ versus $s . C_{1}(2,2)$ bifurcates from $O P C$ at $s_{c, o p c}=4.4517$. At $s \approx 7.6$ another $(2,2)$-branch (not shown) is created in a saddle node bifurcation and persists for $s$ above the saddle node value. $C_{1}(1,3)$ and $C_{\varepsilon}(1,3)$ undergo a transcritical bifurcation at $s_{c, 13}=3.1017$. 
(a)
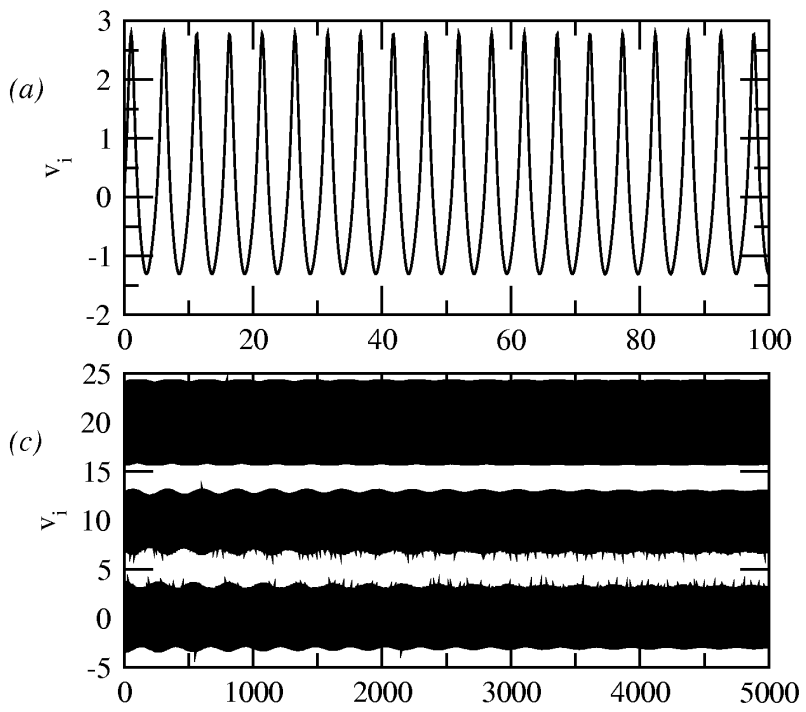

(e)

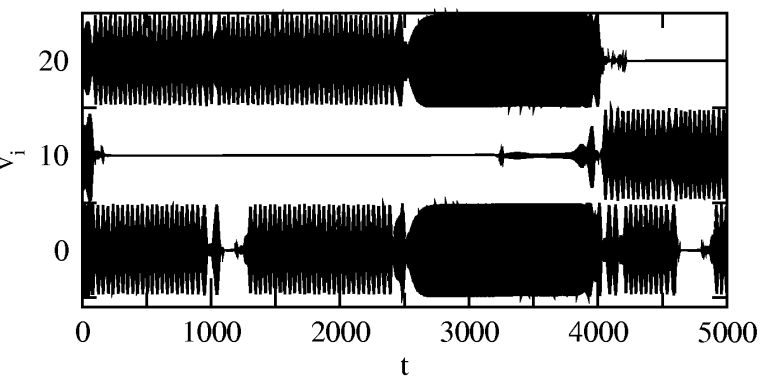

(b)

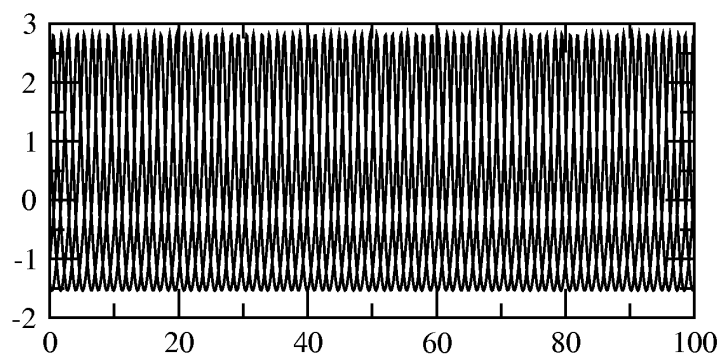

(d)
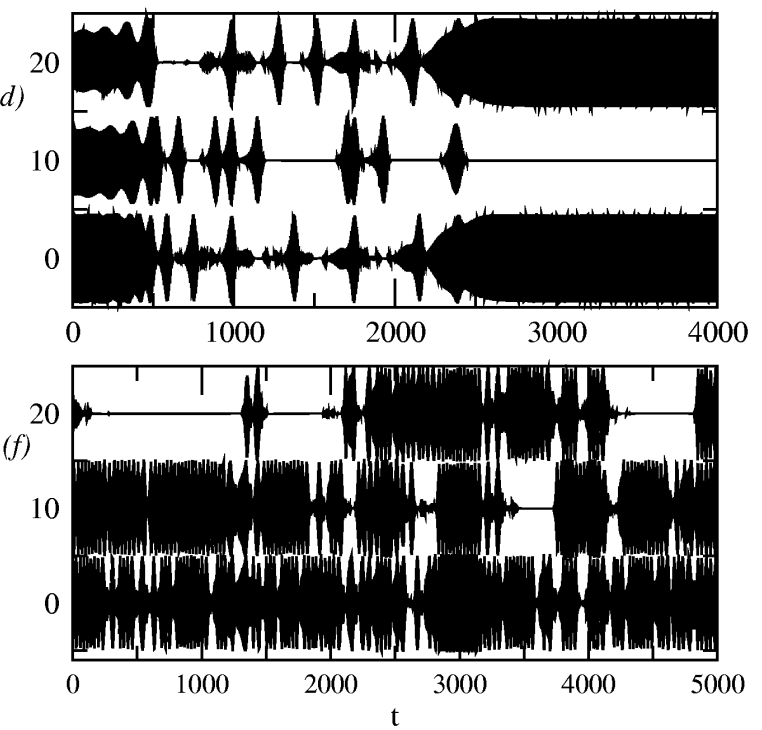

Figure 13: Simulations for the four coupled oscillators with CDO, $c_{1}=c_{3}=0, \varepsilon=0.05$ and $s=1.8$. For $(a)-(f)$ we have set $c_{2}=0.2,-0.1,-0.11,-0.2,-0.4,-0.5$, respectively. For $(a)$, $(b)$ we plot the positions $v_{i}$ against $t$ while in $(c)$ - $(f)$ we plot the differences $v_{1}-v_{k}+10(k-2)$ for $k=2,3,4$, and so a flat part of the plot indicates that two oscillators are synchronized during this time. Observe that (a) shows attracting UO, (b) attracting $R W,(c)$ attracting (2,1,1), while (d)-(f) show attractors that visit a range of different symmetries over a long timespan.

$g\left(\theta_{j}, \theta_{i}\right)$. The $\mathbf{S}_{n}$-symmetry can be removed by considering $(i, j)$-dependent functions $f_{i j}\left(\theta_{j}-\theta_{i}\right)$ and $g_{i j}\left(\theta_{j}-\theta_{i}\right)$. The term $-\beta \alpha_{i}$ in (38) is necessary to stabilize the oscillations.

The system (38) is a simplified version of the reduced system derived in [6] for weakly perturbed Hamiltonian oscillators. Global coupling is via functions $f$ and $g$ of the phase differences. These coupling functions are general $2 \pi$-periodic functions. As with the weakly dissipative oscillators discussed in Section 1.1, we can distinguish some special cases; if $f \not \equiv 0, g \equiv 0$ we say there is coupling through the derivatives only: $\mathrm{CDO}$; if $f \equiv 0, g \not \equiv 0$ we say there is coupling through positions only: CPO.

The equation (38) can be seen as a model for the dynamics of globally coupled oscillators where one cannot necessarily complete a reduction to pure phase oscillators. This will occur for example if there is one Floquet multiplier close to unity corresponding to a direction transverse to the torus. For this reason, we believe the model (38) will be useful in examining effects such as oscillator death [11] where oscillators cease to oscillate, as well as enable us to observe torus breakup, "ponies on a merry-go-round' solutions [2] and other effects in quite a general setting. 

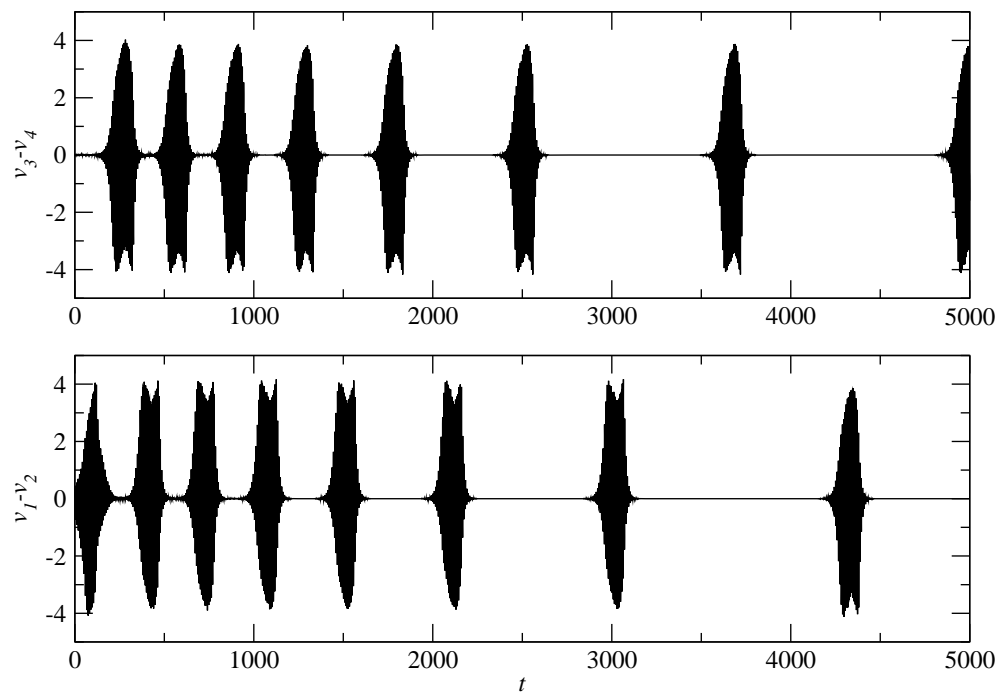

Figure 14: Simulations for the four coupled oscillators with $c_{1}=0.04, c_{2}=0.1, c_{3}=0, \varepsilon=0.8$ and $s=1.8$. The differences of $v_{1}-v_{2}$ and $v_{3}-v_{4}$ are shown and there is clearly an attracting structurally stable heteroclinic cycle between cluster states with symmetry $\mathbf{S}_{2} \times \mathbf{S}_{2}$ where $v_{1}=v_{2}$ and $v_{3}=v_{4}$.

Periodic solutions of the phase energy model Periodic solutions of (38) of a variety of types, such as the uniform oscillations UO, rotating waves RW and cluster states can be located by taking advantage of the symmetry (40). There will be periodic solutions with $\alpha_{i}=A$ constant and $\theta_{i}(t)=\Omega t+\delta_{i}$, where $\Omega=\omega(A)$ (although there can also be periodic solutions with $O(1)$ energy differences as discussed in Section 2.4). Constants $A$ and $\delta_{i}$ can be found such that the equations

$$
\begin{aligned}
\Omega & =\omega(A)+\frac{\varepsilon}{n} \sum_{j=1}^{n} f\left(\delta_{j}-\delta_{i}\right) \\
0 & =-\beta\left(A-\alpha_{0}\right)+\frac{\varepsilon}{n} \sum_{j=1}^{n} g\left(\delta_{j}-\delta_{i}\right), \quad i=1, \cdots, n
\end{aligned}
$$

are satisfied. The equations (41) are overdetermined (there are $2 n$ equations with $n+1$ unknowns) in the general case, and we believe this means that in general periodic orbits will be hard to find for $\varepsilon$ small but non-zero. However, if we consider only coupling through position $f \equiv 0$ then the first $n$ equations are identical and so we have $n+1$ equations with $n+1$ unknowns.

\subsection{Reduction to a phase-only model}

The case of only coupling through the positions clearly leads to a trivial reduction to a Kuramototype phase oscillator of the form

$$
\dot{\theta}_{i}=\omega\left(\alpha_{0}\right)+\frac{\varepsilon}{n} \sum_{j} f\left(\theta_{j}-\theta_{i}\right)
$$

as the $\alpha_{i}$ will simply decay to $\alpha_{0}$. This can be generalized to a phase reduction if the evolution of the phase differences occurs over a timescale much slower than that of the energies. More precisely, if we replace the second equation in (38) by

$$
0=-\beta\left(\alpha_{i}-\alpha_{0}\right)+\frac{\varepsilon}{n} \sum_{j=1}^{n} g\left(\theta_{j}-\theta_{i}\right)
$$


then we can solve

$$
\alpha_{i}=\frac{\varepsilon}{n \beta} \sum_{j=1}^{n} g\left(\theta_{j}-\theta_{i}\right)+\alpha_{0}
$$

where this is justifiable in the limit $\varepsilon \rightarrow 0$ if $\varepsilon / \beta=o(1)$, corresponding to the case of coupling weaker than dissipation considered in [6]. This gives coupled phase equations of the form

$$
\dot{\theta}_{i}=\omega\left(\alpha_{0}+\frac{\varepsilon}{n \beta} \sum_{j} g\left(\theta_{j}-\theta_{i}\right)\right)+\frac{\varepsilon}{n} \sum_{j=1}^{n} f\left(\theta_{j}-\theta_{i}\right) .
$$

Hence to first order in $\varepsilon$ we have

$$
\dot{\theta}_{i}=\omega\left(\alpha_{0}\right)+\frac{\varepsilon}{n} \sum_{j=1}^{n} G\left(\theta_{j}-\theta_{i}\right)+O\left(\varepsilon^{2}\right),
$$

with

$$
G(\theta)=\frac{\omega^{\prime}\left(\alpha_{0}\right)}{\beta} g(\theta)+f(\theta) .
$$

This adiabatic reduction clearly does not need any special assumptions on $f$ and $g$. On the other hand, if $\varepsilon / \beta=O(1)$ corresponding to the case of coupling and dissipation of the same order as considered in this paper, the reduction to a phase-only model is not possible. In fact an interesting feature of this model is precisely that it can be used even in regions where the reduction to phase oscillators is not possible.

\subsection{Example: four globally coupled phase-energy oscillators}

We consider a system of $n=4$ globally coupled oscillators (with coupling of the type CPO), of the form (38) with $f \equiv 0$ and

$$
g(\phi)=\sin (\phi+\delta)+r \sin (2 \phi) .
$$

This choice was inspired by the phase oscillator system of Hansel et al. [14] and studied by Kori and Kuramoto [19]. This provides an interesting example of a system of phase oscillators with an attracting robust heteroclinic cycle between $\mathbf{S}_{2} \times \mathbf{S}_{2}$ cluster states. For the model (38) we can reproduce the behaviour observed in Hansel et al [14] even though our model consists of two degree of freedom oscillators. More precisely,

$$
r=0.25, \quad \delta=4.391, \quad \varepsilon=0.125, \quad \lambda_{1}=-0.4, \quad \lambda_{2}=0, \quad \beta=1, \quad \alpha_{0}=0 \text { and } n=4 .
$$

For these values we can solve (41) and observe that in the invariant subspace $\mathbf{S}_{2} \times \mathbf{S}_{2}$ there are periodic orbits with $0=\delta_{1}=\delta_{2}=\delta_{3}+0.8869=\delta_{4}+0.8869$ and $\alpha_{i}=-0.096763$. There are of course other symmetrically related orbits. Note that the location of the orbits is independent of the $\lambda_{i}$ and observe from the trajectories in Figure 15 that there is a robust heteroclinic attracting cycle between orbits of this form, as observed in the phase oscillator model of [14]. This confirms that the existence of such cycles in globally coupled systems is not just limited to phase oscillator models.

By setting $\lambda_{2} \neq 0$ and changing the parameter one can observe change of isochronicity in the system (38), along with the associated changes in stability, however, one should note that because the location of periodic solutions as given by (41) is independent of the $\lambda_{i}$, the bifurcations can only occur without change in the number and location of periodic solutions; bifurcations can be observed on varying the system parameters. For instance, if we choose the system and parameters 

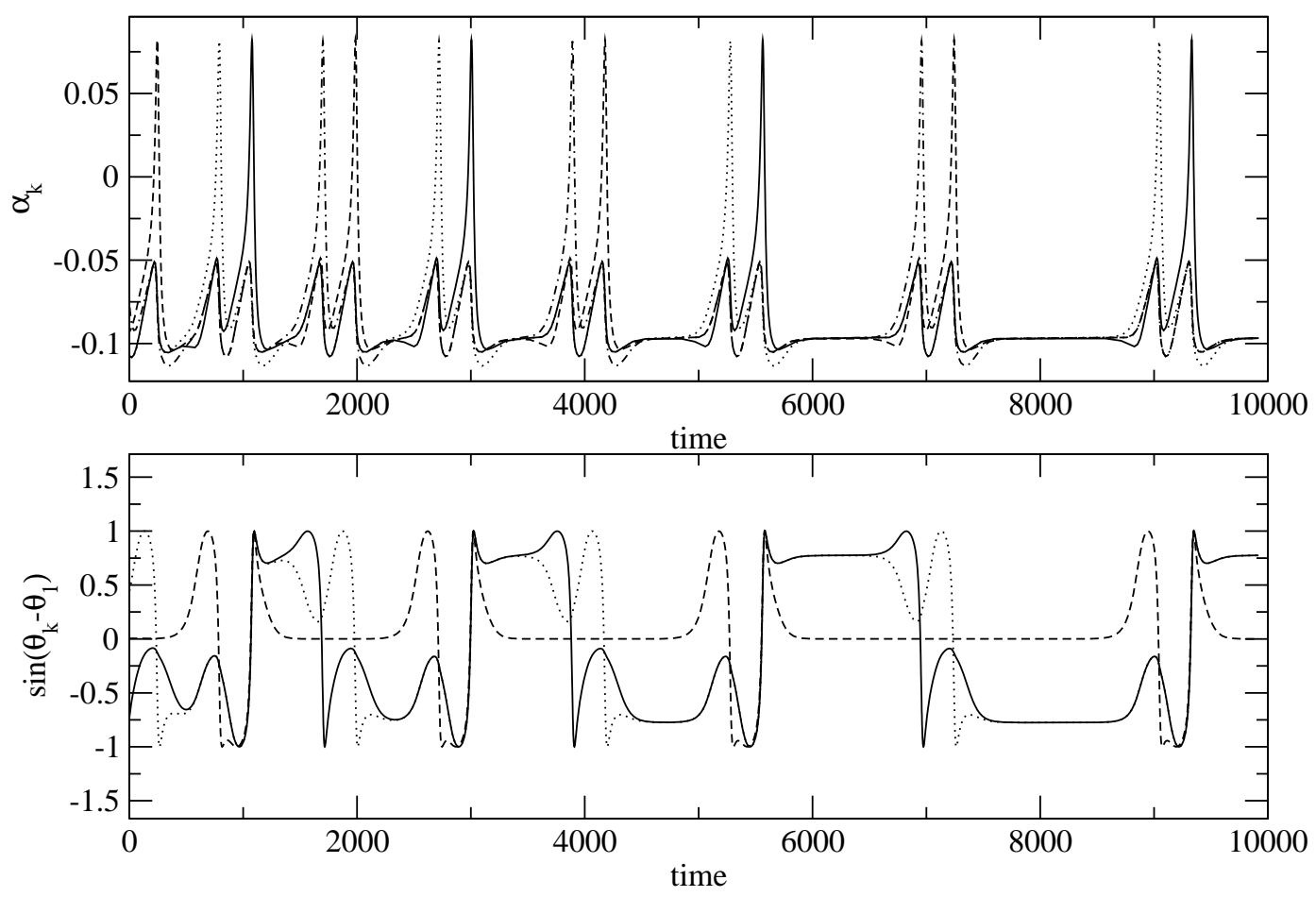

Figure 15: The energies $\alpha_{k}$ and $\sin \left(\theta_{k}-\theta_{1}\right)$ for the phase-energy model (38) with parameters that give a structurally stable heteroclinic cycle in $n=4$ oscillators between $\mathbf{S}_{2} \times \mathbf{S}_{2}$ cluster oscillations. Observe that as time progresses, the phase differences are synchronized in pairs for most of the time, except during events that represent motion close to a heteroclinic connection.

as in (38) and (42) other than changing the parameters to $\lambda_{1}=0, \lambda_{2}=-1$ we find on increasing $\alpha_{0}$ from -0.2 stable $\left(\mathbf{S}_{2}\right)^{2} \times{ }_{s} \mathbf{Z}_{2}$ periodic orbits with

$$
\theta_{1}=\theta_{2}=\theta_{3}+\pi=\theta_{4}+\pi
$$

then $\mathbf{S}_{3}$ cluster periodic orbits, then heteroclinic cycles between $\mathbf{S}_{2} \times \mathbf{S}_{2}$. This is illustrated in Figure 16(a-c); for (a-c) we have set $\alpha=-0.2,0.0,0.2$ respectively. The final timeseries (d) of this figure shows an example of a chaotic attractor for $\lambda_{1}=2, \lambda_{2}=1, \beta=0.1, \alpha_{0}=1, \varepsilon=0.6$ and other parameters as in (42). This has periods where one oscillator undergoes several revolutions while another remains more or less stationary or even reverses its direction.

\section{Conclusions}

In summary, we have extended the analysis in [6] to examine a class of $n$ weakly dissipative globally coupled oscillators where the coupling may be comparable to the dissipation, as well as examining a much larger range of solutions in this system. Although we can no longer guarantee the existence of a normally hyperbolic invariant torus, we can still locate and examine the stability of several classes of periodic solutions. The assumption of $\mathbf{S}_{n}$ symmetry introduced by the global coupling is important in forcing a number of periodic solutions to exist in two and four-dimensional invariant subspaces of the phase space. 

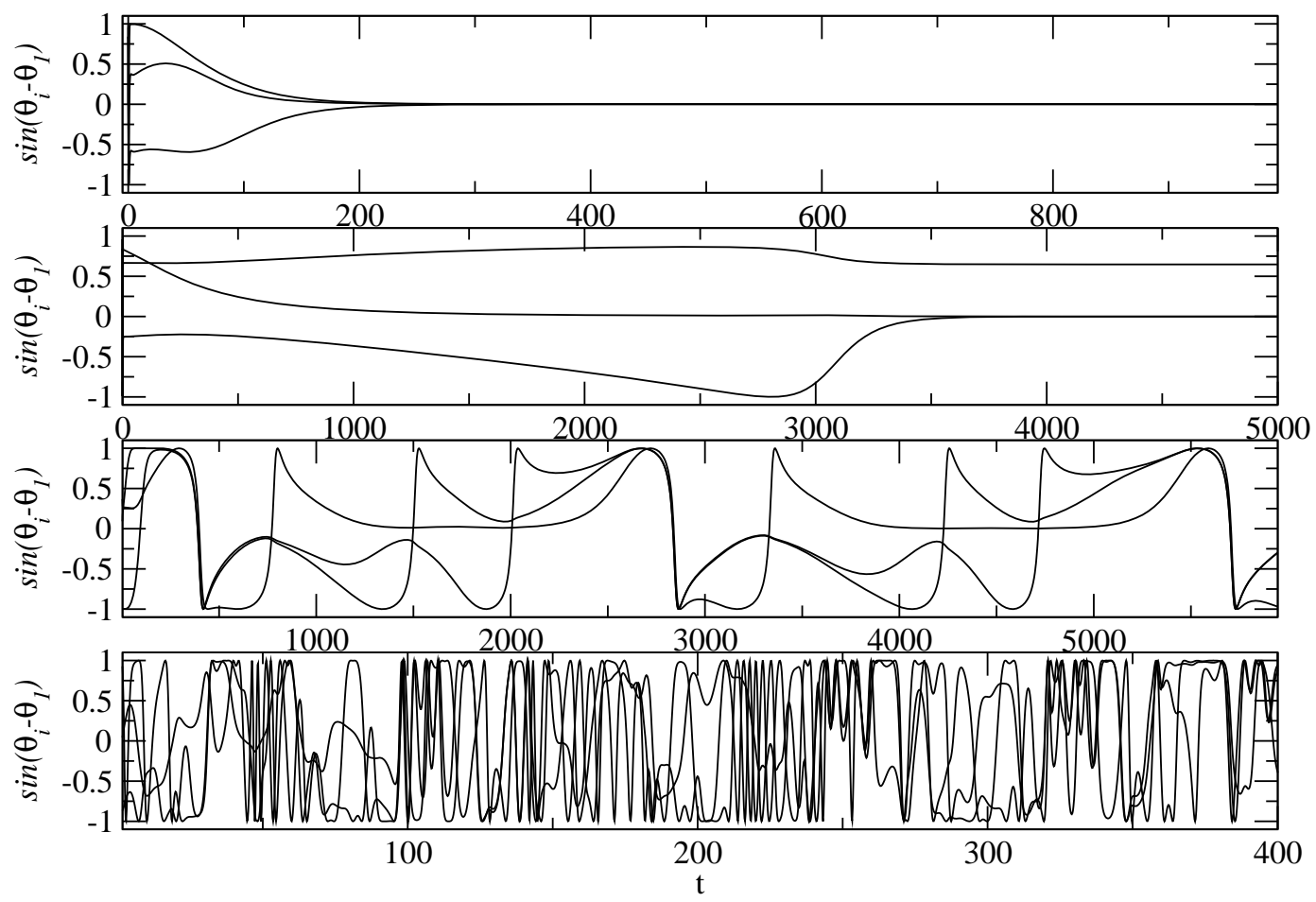

Figure 16: Plots of $\sin \left(\theta_{i}-\theta_{1}\right), i=2,3,4$ for the phase-energy model (38) with different parameters (see text). (a) show convergence to a periodic orbit that on closer inspection has symmetry $\left(\mathbf{S}_{2} \times\right.$ $\left.\mathbf{S}_{2}\right) \times{ }_{s} \mathbf{Z}_{2}$, (b) shows convergence to a periodic solution with symmetry $\mathbf{S}_{3}$, (c) shows the early stages of convergence towards an attracting heteroclinic cycle between $\mathbf{S}_{2} \times \mathbf{S}_{2}$ states, while (d) shows an apparently chaotic trajectory.

In our averaging analysis we have concentrated on periodic solutions for which the frequencies of all oscillators are identical, i.e., on the case of a $(1,1, \ldots, 1)$ resonance. The averaging approach of Section 2 can be applied to other resonances as well, and the presence of isochronicity leads also here to resonance sets consisting of several sheets giving rise to a high multiplicity of oscillating solutions.

The simple phase-energy model introduced in Section 4 is inspired by the form of (6). This can also be seen as being analogous to a system of voltage controlled oscillators (VCOs) of the form investigated in [15], where there is a turning point in the relation between frequency and input voltage. There is work in progress examining the dynamics of (38), examining in particular passage through isochronicity for $n>4$ and also behaviour in the system.

Finally, we would like to highlight that the special types of coupling CDO, CPO and SCD are all 'atypical' in one way or another and hence couplings of these types are likely to miss phenomena that are generic in systems of nonlinearly coupled nonlinear oscillators. Mixed linear coupling (Section 3.3) is in fact 'more typical' and hence we would recommend this for general phenomenological models. 


\section{Acknowledgements}

We thank the London Mathematical Society for providing funds for GD to visit PA in Exeter during spring of 2003 and the Leverhulme Foundation for partial support of PA during writing up. We thank Frank Hoppensteadt and Jon Borresen for conversations related to this work, and the referee for helpful comments regarding the organization of this paper.

\section{References}

[1] D.G. Aronson, G.B. Ermentrout, and N. Kopell. Amplitude response of coupled oscillators. Physica D, 41:403-449, 1990.

[2] D.G. Aronson, M. Krupa and P. Ashwin, Semirotors in the Josephson junction equations. Journal of Nonlinear Science, 6:85-103, 1996.

[3] P. Ashwin and J.W. Swift. The dynamics of $n$ identical oscillators with symmetric coupling. Journal of Nonlinear Science, 2:69-108, 1992.

[4] P. Ashwin. Symmetric chaos in systems of three and four coupled oscillators, Nonlinearity 4:604-618, 1990.

[5] P. Ashwin. Weak coupling of strongly nonlinear, weakly dissipative identical oscillators, $D y$ namics and Stability of Systems 10:203-216, 1995.

[6] P. Ashwin and G. Dangelmayr. Unfolding isochronicity in weakly dissipative coupled oscillators. Dynamics and Stability of Systems 15:263-286, 2000.

[7] N.N. Bogoliubov. Asymptotic methods in nonlinear oscillations. Hindustan Pub. Corp., Delhi, 1961.

[8] F.J. Bourland, R. Haberman, and W.L. Kath. Averaging methods for the phase shift of arbitrarily perturbed strongly nonlinear oscillators with an application to capture. SIAM J. Appl. Math., 51:1150-1167, 1991.

[9] S.-N. Chow and J. Sanders. On the number of critical points of the period. J. Differential Equations 64:51-66, 1986.

[10] G. Dangelmayr and M. Kirby. On diffusively coupled oscillators. In E. Allgower, K. Böhmer, and M. Golubitsky, editors, Bifurcation and Symmetry, Marburg 1991, volume 104 of ISNM, pages 85-97. Birkhäuser, Basel, 1992.

[11] G.B. Ermentrout. Oscillator death in populations of 'all to all' coupled nonlinear oscillators. Physica D 41:219-231, 1990.

[12] G.B. Ermentrout. XPPAUT-The Differential Equations Tool. Available at ftp://ftp.math.pitt.edu.au/pub/bardware/ (1994-).

[13] M. Golubitsky and I. Stewart. Singularities and Groups in Bifurcation Theory, Vol. II. Springer-Verlag, New York, 1988.

[14] D. Hansel, G. Mato and C. Meunier. Clustering and slow switching in globally coupled phase oscillators. Phys. Rev. E 48:3470-3477, 1993. 
[15] F.C. Hoppensteadt and E.M. Izhikevich. Weakly connected neural networks. Volume 126 of App. Math. Sci. Springer, New York, 1997.

[16] J. Kevorkian. Perturbation techniques for oscillatory systems with slowly varying coefficients. SIAM Review, 29:391-461, 1987.

[17] J. Kevorkian and J.D. Cole. Perturbation methods in applied mathematics, volume 34 of App. Math. Sci. Springer, New York, 1981.

[18] Y. Kuramoto. Chemical oscillations, waves, and turbulence Springer, New York, 1984.

[19] H. Kori and Y. Kuramoto. Slow switching in globally coupled oscillators: robustness and occurrence through delayed coupling. Phys. Rev. E 63:046214, 2001.

[20] J.C. Luke. A perturbation method for nonlinear dispersive wave problems. Proc. R. Soc. London A, 292:403-412, 1965. 


\section{A Appendix}

\section{A.1 Details of averaging calculations for the uniform oscillation}

\section{A.1.1 Stability coefficients for generic coupling}

To find the matrices $A, B$ defined in Section 2.1.2, we have to compute the $O(\varepsilon)$-corrections $\phi_{1}^{(1)}(\varphi)$, $\beta_{1}^{(1)}(\varphi)$ in the solution (13) of the linear system that results from linearization of (6) about the uniform oscillation. Setting $\left(\psi_{i}, \alpha_{i}\right)=\left(\psi_{r}+\phi_{i}, \alpha_{r}+\beta_{i}\right)$ and expanding (6) in $\left(\phi_{i}, \beta_{i}\right)$ up to linear order yields a system of the form

$$
\begin{aligned}
\frac{d \phi_{i}}{d t} & =\omega^{\prime} \beta_{i}+\varepsilon\left(a_{1} \phi_{i}+b_{1} \beta_{i}+c_{1} \sum_{j \neq i} \phi_{j}+d_{1} \sum_{j \neq i} \beta_{j}\right)+O\left(\varepsilon^{2}\right), \\
\frac{d \beta_{i}}{d t} & =\varepsilon\left(a_{2} \phi_{i}+b_{2} \beta_{i}+c_{2} \sum_{j \neq i} \phi_{j}+d_{2} \sum_{j \neq i} \beta_{j}\right)+O\left(\varepsilon^{2}\right),
\end{aligned}
$$

where $a_{i}, b_{i}, c_{i}, d_{i}$ are functions of $\varphi$ defined through partial derivatives of the $O(\varepsilon)$-terms in (6). We use $\varphi$ as independent variable and rewrite this system as

$$
\begin{aligned}
\omega \dot{\phi}_{i} & =\omega^{\prime} \beta_{i}+\varepsilon\left(a_{1} \phi_{i}+\tilde{b}_{1} \beta_{i}+c_{1} \sum_{j \neq i} \phi_{j}+d_{1} \sum_{j \neq i} \beta_{j}\right)+O\left(\varepsilon^{2}\right), \\
\omega \dot{\beta}_{i} & =\varepsilon\left(a_{2} \phi_{i}+b_{2} \beta_{i}+c_{2} \sum_{j \neq i} \phi_{j}+d_{2} \sum_{j \neq i} \beta_{j}\right)+O\left(\varepsilon^{2}\right),
\end{aligned}
$$

where $\tilde{b}_{1}=b_{1}-\Omega^{(1)} \omega^{\prime} / \omega$, and all functions of $(\varphi, \alpha)$ can be evaluated at $\alpha=\alpha^{(0)}$ if, as we do, $O\left(\varepsilon^{2}\right)$-terms are neglected. A regular perturbation analysis shows that the first order terms $\phi_{i}^{(1)}(\varphi), \beta_{i}^{(1)}(\varphi)$ in (13) satisfy

$$
\begin{aligned}
& \omega \dot{\phi}_{i}^{(1)}=\omega^{\prime} \beta_{i}^{(1)}+a_{1}\left(\frac{\omega^{\prime}}{\omega} \beta_{i 0} \varphi+\phi_{i 0}\right)+\tilde{b}_{1} \beta_{i 0}+c_{1} \sum_{j \neq i}\left(\frac{\omega^{\prime}}{\omega} \beta_{j 0} \varphi+\phi_{j 0}\right)+d_{1} \sum_{j \neq i} \beta_{j 0}, \\
& \omega \dot{\beta}_{i}^{(1)}=a_{2}\left(\frac{\omega^{\prime}}{\omega} \beta_{i 0} \varphi+\phi_{i 0}\right)+b_{2} \beta_{i 0}+c_{2} \sum_{j \neq i}\left(\frac{\omega^{\prime}}{\omega} \beta_{j 0} \varphi+\phi_{j 0}\right)+d_{2} \sum_{j \neq i} \beta_{j 0},
\end{aligned}
$$

with $\left(\phi_{i 0}, \beta_{i 0}\right)$ the initial values. The solution for $\beta_{i}^{(1)}$ is

$$
\begin{aligned}
\omega \beta_{i}^{(1)}= & \left(\int_{0}^{\varphi}\left(\frac{\omega^{\prime}}{\omega} a_{2} \varphi^{\prime}+b_{2}\right) d \varphi^{\prime}\right) \beta_{i 0}+\left(\int_{0}^{\varphi} a_{2} d \varphi^{\prime}\right) \phi_{i 0}+ \\
& \left(\int_{0}^{\varphi}\left(\frac{\omega^{\prime}}{\omega} c_{2}+d_{2}\right) \varphi^{\prime} d \varphi^{\prime}\right) \sum_{j \neq i} \beta_{j 0}+\left(\int_{0}^{\varphi} c_{2} d \varphi^{\prime}\right) \sum_{j \neq i} \phi_{j 0} .
\end{aligned}
$$

We denote the entries of the matrices $A$ and $B$ by $a_{i j}$ and $b_{i j}$. The entries $b_{21}, b_{22}, a_{21}$ and $a_{22}$ are the coefficients of $\phi_{i 0}, \beta_{i 0}, \sum_{j \neq i} \phi_{j 0}$ and $\sum_{j \neq i} \beta_{j 0}$ in $\beta_{i}^{(1)}(2 \pi)$, respectively, i.e.,

$$
\begin{aligned}
\omega b_{21} & =\int_{0}^{2 \pi} a_{2}(\varphi) d \varphi, & \omega b_{22} & =\int_{0}^{2 \pi}\left(\frac{\omega^{\prime}}{\omega} a_{2}(\varphi) \varphi+b_{2}(\varphi)\right) d \varphi \\
\omega a_{21} & =\int_{0}^{2 \pi} c_{2}(\varphi) d \varphi, & \omega a_{22} & =\int_{0}^{2 \pi}\left(\frac{\omega^{\prime}}{\omega} c_{2}(\varphi) \varphi+d_{2}(\varphi)\right) d \varphi .
\end{aligned}
$$

Similarly, the entries $b_{11}$ and $a_{11}$ are the coefficients of $\phi_{i 0}$ and $\sum_{j \neq i} \phi_{j 0}$ in $\phi_{i}^{(1)}(2 \pi)\left(a_{12}\right.$ and $b_{12}$ are not needed). By substituting $\beta_{i}^{(1)}(\varphi)$ in the equation for $\dot{\phi}_{i}^{(1)}$ and integrating from 0 to $2 \pi$ we 
obtain

$$
\begin{aligned}
\omega b_{11} & =\frac{\omega^{\prime}}{\omega} \int_{0}^{2 \pi} \int_{0}^{\varphi} a_{2}\left(\varphi^{\prime}\right) d \varphi^{\prime} d \varphi+\int_{0}^{2 \pi} a_{1}(\varphi) d \varphi, \\
\omega a_{11} & =\frac{\omega^{\prime}}{\omega} \int_{0}^{2 \pi} \int_{0}^{\varphi} c_{2}\left(\varphi^{\prime}\right) d \varphi^{\prime} d \varphi+\int_{0}^{2 \pi} c_{1}(\varphi) d \varphi .
\end{aligned}
$$

Thus only the functions $a_{1}, a_{2}, b_{2}, c_{1}, c_{2}, d_{2}$ are required. A straightforward computation yields

$$
\begin{aligned}
a_{1} & =-\omega\left(z^{\prime} f\right)_{, \varphi}+(n-1) \omega z^{\prime}\left(\dot{z} q_{, v_{2}}+\omega \ddot{z} q_{, u_{2}}\right), \\
a_{2} & =\omega(\dot{z} f)_{, \varphi}-(n-1) \omega \dot{z}\left(\dot{z} q_{v_{2}}+\omega \ddot{z} q_{, u_{2}}\right), \\
b_{2} & =(\omega \dot{z} f)^{\prime}-(n-1) \omega \dot{z}\left(z^{\prime} q_{, v_{2}}+(\omega \dot{z})^{\prime} q_{, u_{2}}\right), \\
c_{1} & =-\omega z^{\prime}\left(\dot{z} q_{, v_{2}}+\omega \ddot{z} q_{, u_{2}}\right), \\
c_{2} & =\omega \dot{z}\left(\dot{z} q_{v_{2}}+\omega \ddot{z} q_{, u_{2}}\right), \\
d_{2} & =\omega \dot{z}\left(z^{\prime} q_{v_{2}}+(\omega \dot{z})^{\prime} q_{u_{2}}\right),
\end{aligned}
$$

with the partial derivatives of $q\left(v_{1}, u_{1}, v_{2}, u_{2}\right)$ evaluated at $v_{1}=v_{2}=z, u_{1}=u_{2}=\omega \dot{z}$. With these expressions the integrals for the matrix entries can, using some partial integrations, be rewritten as averages over $2 \pi$-periodic functions as follows:

$$
\begin{aligned}
& a_{11}=-2 \pi \overline{z^{\prime} d}+\pi \omega^{\prime} T \overline{\dot{z} d}+\omega^{\prime} T \bar{D} \\
& a_{21}=2 \pi \overline{\dot{z} d} \\
& a_{22}=2 \pi \dot{\dot{z} e}+\pi \omega^{\prime} T \overline{\dot{z} d}-\omega^{\prime} T \bar{D} \\
& b_{11}=2 \pi(n-1) \overline{z^{\prime} d}-\omega^{\prime} T(n-1)(\pi \overline{\dot{z} d}+\bar{D})-\left.\omega^{\prime} T(\dot{z} f)\right|_{\varphi=0} \\
& b_{21}=-2 \pi(n-1) \overline{\dot{z} d} \\
& b_{22}=2 \pi \overline{(\dot{z} f)^{\prime}}-2 \pi(n-1) \overline{\dot{z} e}-\omega^{\prime} T(n-1)(\pi \overline{\dot{z} d}-\bar{D})+\left.\omega^{\prime} T(\dot{z} f)\right|_{\varphi=0},
\end{aligned}
$$

where

$$
d=q_{, v_{2}} \dot{z}+q_{, u_{2}} \omega \ddot{z}, \quad e=q_{, v_{2}} z^{\prime}+q_{,_{2}}(\omega \dot{z})^{\prime}, \quad D=\int_{0}^{\varphi}(\dot{z} d-\bar{z} d) d \varphi .
$$

\section{A.1.2 Derivation of the system (16)}

We assume here that the leading order coupling function depends on the derivatives only, $q=$ $q\left(u_{1}, u_{2}\right)$. As in Section 2.1, all functions of $\left(v_{i}, u_{i}\right)$ are considered as functions of $(\varphi, \alpha)$ via the substitution $\left(v_{i}, u_{i}\right)=(z, \omega \dot{z})$, and are evaluated at $\alpha=\alpha^{(0)}$, the persistence energy determined by equation (10). We set

$$
S(u)=\int u q_{, u_{2}}(u, u) d u .
$$

When the expansion (15) is substituted in (6), the $\alpha_{i}$-equation at $O\left(\varepsilon^{2}\right)$ gives

$$
\omega \dot{\beta}_{i}^{(2)}=\omega(\dot{z} f)_{, \varphi} \phi_{i}+\dot{S} \sum_{j}\left(\phi_{j}-\phi_{i}\right)
$$

with $f$ defined below (8). Since the average of the right hand side of this equation vanishes, no solvability condition has to be imposed at this stage. Note that the second term on the right hand side becomes a $\varphi$-derivative only if there is coupling in the derivatives only. The (zero-mean) solution for $\beta_{i}^{(2)}$ is

$$
\beta_{i}^{(2)}=(\dot{z} f) \phi_{i}+(1 / \omega)(S-\bar{S}) \sum_{j}\left(\phi_{j}-\phi_{i}\right) .
$$


At $O\left(\varepsilon^{2}\right)$ in the $\psi_{i}$-equation we find

$$
\omega \dot{\psi}_{i}^{(2)}=-\phi_{i, \tau}+\omega^{\prime}\left(e_{i}+\beta_{i}^{(2)}\right)-\omega\left(z^{\prime} f\right)_{, \varphi} \phi_{i}-\omega^{2} z^{\prime} \ddot{z} q_{u_{2}} \sum_{j}\left(\phi_{j}-\phi_{i}\right) .
$$

The solvability condition for $\psi_{i}^{(2)}$ leads directly to the first equation in (16).

To find the equation for $e_{i}$ we have to examine the equation for $\alpha_{i}$ at $O\left(\varepsilon^{3}\right)$. The resulting equation for $\beta_{i}^{(3)}$ has the form

$$
\begin{aligned}
\omega \dot{\beta}_{i}^{(3)}= & -e_{i, \tau}+A \phi_{i}+(\omega \dot{z} f)_{, \varphi} \psi_{i}^{(2)}+(\omega \dot{z} f)^{\prime}\left(e_{i}+\beta_{i}^{(2)}\right)+\sum_{j}\left(B\left(\phi_{j}-\phi_{i}\right)\right. \\
& \left.+C\left(\phi_{j}-\phi_{i}\right)^{2}+\dot{S}\left(\psi_{j}^{(2)}-\psi_{i}^{(2)}\right)+S^{\prime}\left(e_{j}-e_{i}+\beta_{j}^{(2)}-\beta_{i}^{(2)}\right)\right)+G,
\end{aligned}
$$

where

$$
\begin{aligned}
A & =\beta^{(1)}(\omega \dot{z} f)_{, \varphi \alpha}+\omega \phi^{(1)}(\dot{z} f)_{, \varphi \varphi}, \\
B & =\beta^{(1)} \dot{S}^{\prime}+\phi^{(1)} \ddot{S}+\omega \dot{z}\left(\dot{z} q_{2, v_{2}}+\omega \ddot{z} q_{2, u_{2}}\right) \\
C & =\left(\omega^{2} / 2\right) \dot{z}\left(\ddot{z} q_{, u_{2}}+\omega \ddot{z}^{2} q_{, u_{2} u_{2}}\right)
\end{aligned}
$$

and

$$
\begin{aligned}
& G=\left(\alpha^{(1)}(\omega \dot{z} f)_{, \varphi \alpha}+\omega(\dot{z} g)_{, \varphi}\right) \phi_{i}+(\omega / 2)(\dot{z} f)_{, \varphi \varphi} \phi_{i}^{2}+\alpha^{(1)} \dot{S}^{\prime} \\
&+(\omega \dot{z} f)_{, \varphi} \phi_{i}^{(2)}+\sum_{j}\left(\ddot{S} \phi_{i}\left(\phi_{j}-\phi_{i}\right)+\dot{S}\left(\phi_{j}^{(2)}-\phi_{i}^{(2)}\right)\right)
\end{aligned}
$$

consists only of terms with zero averages, i.e. $\bar{G}=0$. Note that the last term in $B$, defined in terms of $q_{2}$, is a $\varphi$-derivative if $q_{2}$ also depends only on the derivatives.

The equation for $e_{i}$ follows by averaging the right hand side of the equation for $\beta_{i}^{(3)}$. To obtain the final form of this equation, we combine various terms as follows:

$$
\begin{aligned}
& \omega \overline{(\dot{z} f)_{, \varphi} \psi_{i}^{(2)}}=-\omega \overline{\dot{z} f \dot{\psi}_{i}^{(2)}} \\
& =-\omega^{\prime} \overline{\dot{z} f \beta_{i}^{(2)}}+\omega \overline{\dot{z} f\left(z^{\prime} f\right)_{, \varphi}} \phi_{i}+\overline{z^{\prime} f \dot{S}} K \\
& =\left(\omega \overline{\dot{z} f\left(z^{\prime} f\right)_{, \varphi}}-\omega^{\prime} \overline{(\dot{z} f)^{2}}\right) \phi_{i}+\left(\overline{z^{\prime} f \dot{S}}-\frac{\omega^{\prime} \overline{\dot{z} f S}}{\omega}\right) K \\
& \bar{A} \phi_{i}+\overline{(\omega \dot{z} f)^{\prime} \beta_{i}^{(2)}}=\left(\omega \overline{\dot{z} f \ddot{\phi}^{(1)}}-\overline{(\omega \dot{z} f)^{\prime} \dot{\beta}^{(1)}}\right) \phi_{i}+\overline{(\omega \dot{z} f)^{\prime} \beta_{i}^{(2)}} \\
& =\left(\omega^{\prime} \overline{(\dot{z} f)^{2}}-\omega \overline{\dot{z} f\left(z^{\prime} f\right)_{, \varphi}}\right) \phi_{i}+\frac{1}{\omega} \overline{(\omega \dot{z} f)^{\prime}(S-\bar{S})} K,
\end{aligned}
$$

where $K \equiv \sum_{j}\left(\phi_{j}-\phi_{i}\right)$, hence

$$
\bar{A} \phi_{i}+\overline{(\omega \dot{z} f)^{\prime} \beta_{i}^{(2)}}+\omega \overline{(\dot{z} f)_{, \varphi} \psi_{i}^{(2)}}=\left(\overline{z^{\prime} f \dot{S}}+\overline{(\dot{z} f)^{\prime}(S-\bar{S})}\right) \sum_{j}\left(\phi_{j}-\phi_{i}\right) .
$$

Similarly,

$$
\sum_{j} \overline{\dot{S}\left(\psi_{j}^{(2)}-\psi_{i}^{(2)}\right)}=-\sum_{j} \overline{S\left(\dot{\psi}_{j}^{(2)}-\dot{\psi}_{i}^{(2)}\right)}
$$




$$
\begin{aligned}
= & \sum_{j}\left(\bar{S}\left(\phi_{j, \tau}-\phi_{i, \tau}\right)-\omega^{\prime} \bar{S}\left(e_{j}-e_{i}\right)-\omega^{\prime} S\left(\beta_{j}^{(2)}-\beta_{i}^{(2)}\right)\right. \\
& \left.+\omega\left(\overline{S\left(z^{\prime} f\right)_{, \varphi}}-n \omega \overline{z^{\prime} \ddot{z} S q_{, u_{2}}}\right)\left(\phi_{j}-\phi_{i}\right)\right) / \omega \\
= & \sum_{j}\left(\overline{S\left(z^{\prime} f\right)_{, \varphi}}\left(\phi_{j}-\phi_{i}\right)-\frac{\omega^{\prime}}{\omega} \overline{S\left(\beta_{j}^{(2)}-\beta_{i}^{(2)}\right)}\right) \\
= & \left(\overline{S\left(z^{\prime} f\right)_{, \varphi}}-\frac{\omega^{\prime} \overline{\dot{z} f S}}{\omega}+\frac{n \omega^{\prime}}{\omega^{2}}\left(\overline{S^{2}}-\bar{S}^{2}\right)\right) \sum_{j}\left(\phi_{j}-\phi_{i}\right), \\
\sum_{j} \overline{S^{\prime}\left(\beta_{j}^{(2)}-\beta_{i}^{(2)}\right)}= & \left(\overline{\dot{z} f S^{\prime}}-\frac{n}{\omega}\left(\overline{S S^{\prime}}-\bar{S} \overline{S^{\prime}}\right)\right) \sum_{j}\left(\phi_{j}-\phi_{i}\right), \\
\bar{B}= & -\overline{\dot{\beta}(1) S^{\prime}}+\overline{\ddot{\phi}(1) S}+\omega \overline{\dot{z}^{2} q_{2, v_{2}}}+\omega^{2} \overline{\dot{z} \ddot{z} q_{2, u_{2}}} \\
= & -\overline{\dot{z} f S^{\prime}}+\frac{\omega^{\prime} \overline{\dot{z} f S}}{\omega}-\overline{S\left(z^{\prime} f\right)_{, \varphi}}+\omega \overline{\dot{z}^{2} q_{2, v_{2}}}+\omega^{2} \overline{\dot{z} \ddot{z} q_{2, u_{2}}} .
\end{aligned}
$$

Combining these terms yields

$\sum_{j}\left(\overline{\dot{S}\left(\psi_{j}^{(2)}-\psi_{i}^{(2)}\right)}+\overline{S^{\prime}\left(\beta_{j}^{(2)}-\beta_{i}^{(2)}\right)}+\bar{B}\left(\phi_{j}-\phi_{i}\right)\right)+\bar{A} \phi_{i}+\overline{(\omega \dot{z} f)^{\prime} \beta_{i}^{(2)}}+\omega \overline{(\dot{z} f)_{, \varphi} \psi_{i}^{(2)}}=P \sum_{j}\left(\phi_{j}-\phi_{i}\right)$,

with $P$ as defined below equation (16). The remaining terms in the equation for $\dot{\beta}_{i}^{(3)}$ lead directly to the coefficients $D, N, Q$.

\section{A.1.3 Periodic solutions of the system (16)}

To analyze (16) we set $\varphi_{i}=\phi_{i}-\phi_{n}, \Delta_{i}=e_{i}-e_{n}(1 \leq i<n)$, and $\phi=\sum_{1}^{n} \phi_{i}, E=\sum_{1}^{n} e_{i}$ and transform the equation to

$$
\begin{aligned}
\frac{d \varphi_{i}}{d \tau} & =\omega^{\prime} \Delta_{i}-n M \varphi_{i} \\
\frac{d \Delta_{i}}{d \tau} & =(D-n N) \Delta_{i}-n P \varphi_{i}+Q\left(n \varphi_{i}-2 \sum_{j=1}^{n-1} \varphi_{j}\right) \varphi_{i}
\end{aligned}
$$

and

$$
\begin{aligned}
\frac{d \phi}{d \tau} & =\omega^{\prime} E \\
\frac{d E}{d \tau} & =D E+2 Q\left(n \sum_{i=1}^{n-1} \varphi_{i}^{2}-\sum_{j, k=1}^{n-1} \varphi_{j} \varphi_{k}\right) .
\end{aligned}
$$

Given an equilibrium $\left(\varphi_{i 0}, \Delta_{i 0}\right)$ of (A.1), then, since $D<0$ is assumed, the total energy $E$ will evolve towards a unique equilibrium value $E_{0}$ when $\varphi=\varphi_{i 0}$ is substituted into (A.2). Together with the differences $\Delta_{i 0}=e_{i 0}-e_{n 0}$ this determines all energy perturbations $e_{i 0}$, and the total phase $\phi$ evolves linearly in $\tau$. Thus the equilibria of (A.1) induce periodic solutions of our original system (1), with frequency $\Omega(\varepsilon)+\varepsilon^{2} \omega^{\prime} E_{0}+O\left(\varepsilon^{3}\right)$. Moreover, the stability of this periodic solution is determined by the eigenvalues of the linearization of (A.1) about $\left(\varphi_{i 0}, \Delta_{i 0}\right)$. The Jacobian of (A.1) is composed of the $2 \times 2$ matrices

$$
J_{i j}=\left[\begin{array}{cc}
\frac{\partial \varphi_{i, \tau}}{\partial \varphi_{j}} & \frac{\partial \varphi_{i, \tau}}{\partial \Delta_{j}} \\
\frac{\partial \Delta_{i, \tau}}{\partial \varphi_{j}} & \frac{\partial \Delta_{i, \tau}}{\partial \Delta_{j}}
\end{array}\right]
$$

whose explicit forms are

$$
J_{i i}=\left[\begin{array}{cc}
-n M & \omega^{\prime} \\
-n P+2 Q(n-2) \varphi_{i}-2 Q \sum_{j \neq i} \varphi_{j} & D-n M
\end{array}\right],
$$


and

$$
J_{i j}=\left[\begin{array}{cc}
0 & 0 \\
-2 Q \varphi_{i} & 0
\end{array}\right] \quad(j \neq i) .
$$

The system (A.1) is $\mathbf{S}_{n-1}$-equivariant, and admits the trivial equilibrium $\varphi_{i}=\Delta_{i}=0$ corresponding to the fully synchronized oscillation determined in Section 2.1.1. Since $\left.J_{i j}\right|_{\{(0,0)\}}$ is the zero matrix if $j \neq i$, the eigenvalues for the trivial equilibrium are those of the matrix

$$
\left.B^{(0)} \equiv J_{i i}\right|_{\{(0,0)\}},
$$

repeated $n-1$ times. Generically the trace and the determinant of this matrix,

$$
\operatorname{Tr} B^{(0)}=D-n(N+M), \quad \operatorname{det} B^{(0)}=n M(D+n N)+n \omega^{\prime} P
$$

will be nonzero and hence will fully determine the stability of the uniform oscillations to perturbations of the form (15).

In addition, (A.1) admits for $n>2$ equilibria of the form $\left(\varphi_{i}, \Delta_{i}\right)=\left(\varphi_{0}^{(m)}, \Delta_{0}^{(m)}\right)$ for $1 \leq i \leq m$, and $\left(\varphi_{i}, \Delta_{i}\right)=(0,0)$ for $m+1 \leq i \leq n-1$, where $1 \leq m \leq n-1, n \neq 2 m$, and

$$
\varphi_{0}^{(m)}=\frac{\operatorname{det} B^{(0)}}{(n-2 m) Q \omega^{\prime}}, \quad \Delta_{0}^{(m)}=\frac{n M}{\omega^{\prime}} \varphi_{0}^{(m)} .
$$

For $m<n-1$ each of these equilibria is a cluster solution, and for $m=n-1$ it is a nontrivial $\mathbf{S}_{n-1}{ }^{-}$ symmetric solution of (A.1), i.e. $\left(\varphi_{i}, \Delta_{i}\right)=\left(\varphi_{0}^{(n-1)}, \Delta_{0}^{(n-1)}\right)$ for $1 \leq i \leq n-1$. The eigenvalues for the cluster solutions (see Appendix A.2.2) are those of the $4 \times 4$ matrix

$$
\left[\begin{array}{cc}
J_{11}+(m-1) J_{12} & (n-1-m) J_{1, m+1} \\
m J_{m+1,1} & J_{m+1, m+1}+(n-m-2) J_{m+1, m+2}
\end{array}\right],
$$

which are simple, and those of the $2 \times 2$ matrices $J_{11}-J_{12}$ and $J_{m+1, m+1}-J_{m+1, m+2}$ which are repeated $m-1$ and $n-m-2$ times, respectively, with $J_{i j}$ evaluated at the equilibrium. Since, for our special system, $J_{m+1,1}$ is the zero matrix, the eigenvalues of the $4 \times 4$ matrix are the eigenvalues of the diagonal blocks. Moreover, $J_{m+1, m+1}=B^{(0)}$ and $J_{m+1, m+2}$ is also the zero matrix, hence the eigenvalues for the equilibria of (A.1), determined by $\left(\varphi_{0}^{(m)}, \Delta_{0}^{(m)}\right)$, are those of the $2 \times 2$ matrices

$$
K^{(m)} \equiv J_{11}+(m-1) J_{12}, \quad L^{(m)} \equiv J_{11}-J_{12}, \quad B^{(0)},
$$

which are simple, repeated $m-1$ times, and repeated $n-1-m$ times, respectively. This includes the $\mathbf{S}_{n-1}$-symmetric case $m=n-1$. A simple calculation shows that

$$
\operatorname{Tr} K^{(m)}=\operatorname{Tr} L^{(m)}=\operatorname{Tr} B^{(0)},
$$

and

$$
\operatorname{det} K^{(m)}=-\operatorname{det} B^{(0)}, \quad \operatorname{det} L^{(m)}=-\frac{n \operatorname{det} B^{(0)}}{n-2 m} .
$$

For each $m$ in the range $1 \leq m \leq n-1, n \neq 2 m$, the oscillation pattern associated with the equilibrium of (A.1), determined by $\left(\varphi_{0}^{(m)}, \Delta_{0}^{(m)}\right)$, is a cluster oscillation, i.e. a solution for which the $n$ oscillators are divided in two fully synchronized groups of $m$ and $n-m$ oscillators, respectively, with a phase shift of order $O(\varepsilon)$ and an energy difference of order $O\left(\varepsilon^{2}\right)$ between the two oscillations. Since for each $m$ there are at least two eigenvalue-determining matrices whose determinants have 
opposite signs (proportional to $\pm \operatorname{det} B^{(0)}$ ), all of these cluster oscillations are saddles. In contrast, the stability of the uniform oscillation is fully determined by $B^{(0)}$.

We expect there will be no degeneracy in the matrix $B^{(0)}$ when $\omega^{\prime}=0$, i.e. we do not expect a stability change of the fully synchronized oscillation when passing through isochronicity. In contrast, the existence of the cluster oscillations required $\omega^{\prime} \neq 0$. We conjecture that passage through isochronicity will lead to bifurcations of these saddle solutions, and that an unfolding of isochronicity [6] will yield further solutions which are not captured by the above analysis.

\section{A.2 Details of averaging calculations for the cluster oscillations}

\section{A.2.1 First order frequency and phase shift corrections}

To find the first order terms $\Omega^{(1)}$ and $\Delta_{\nu}^{(1)}$ we have to examine the energy equation at second order. Analogously as in Section 2.1.1 we denote by $\varepsilon^{2} \beta_{\nu}^{(2)}$ the $2 \pi$-periodic, zero-mean corrections of $\alpha_{\nu}$ at order $O\left(\varepsilon^{2}\right)$. Collecting terms at $O\left(\varepsilon^{2}\right)$ in $d \alpha_{\nu} / d t$ then yields,

$$
\Omega^{(1)} \dot{\beta}_{\nu}^{(1)}+\omega \dot{\beta}_{\nu}^{(2)}=\sum_{\mu=1}^{2}\left(a_{\nu \mu}\left(\alpha_{\mu}^{(1)}+\beta_{\mu}^{(1)}\right)+b_{\nu \mu}\left(\Delta_{\mu}^{(1)}+\phi_{\mu}^{(1)}\right)\right)+\omega \dot{z} g_{\nu},
$$

where for $\mu \neq \nu$,

$$
a_{\nu \mu}=\omega \dot{z} e_{\nu}, \quad a_{\nu \nu}=\left(\omega \dot{z} f_{\nu}\right)^{\prime}-a_{\nu \mu}, \quad b_{\nu \mu}=\omega \dot{z} d_{\nu}, \quad b_{\nu \nu}=\omega\left(\dot{z} f_{\nu}\right)_{, \varphi}-b_{\nu \mu},
$$

and

$$
g_{\nu}=p_{2}(v, u)+\left(m_{\nu}-1\right) q_{2}(v, u, v, u)+m_{\mu} q_{2}\left(v, u, v_{\mu}, u_{\mu}\right)
$$

results from the second order terms of the original system. The solvability condition for $\beta_{\nu}^{(2)}$ leads to the following linear system of equations,

$$
\sum_{\mu=1}^{2} \overline{a_{\nu \mu}} \alpha_{\mu}^{(1)}+(-1)^{\nu} b_{\nu}\left(\Delta_{1}^{(1)}-\Delta_{2}^{(1)}\right)+c_{\nu}=0,
$$

where

$$
c_{\nu}=\sum_{\mu=1}^{2}\left(\overline{a_{\nu \mu} \beta_{\mu}^{(1)}}+\overline{b_{\nu \mu} \phi_{\mu}^{(1)}}\right)+\omega \overline{\dot{z} g_{\nu}}
$$

and $b_{\nu}=\overline{b_{\nu \mu}}=\omega \overline{\dot{z} d_{\nu}}(\mu \neq \nu)$. When $\alpha_{\nu}^{(1)}$ from (27) is substituted in (A.3), we are left with two linear equations for $\Omega^{(1)}$ and the first order phase shift $\Delta_{1}^{(1)}-\Delta_{2}^{(1)}$, with the solution

$$
\begin{aligned}
\Omega^{(1)} & =-\frac{\omega\left(\overline{a_{11}} b_{2}+\overline{a_{21}} b_{1}\right) \overline{z^{\prime} f_{1}}+\omega\left(\overline{a_{12}} b_{2}+\overline{a_{22}} b_{1}\right) \overline{z^{\prime} f_{2}}+\omega^{\prime}\left(c_{1} b_{2}+c_{2} b_{1}\right)}{\left(\overline{a_{11}}+\overline{a_{12}}\right) b_{2}+\left(\overline{a_{21}}+\overline{a_{22}}\right) b_{1}} \\
\Delta_{1}^{(1)}-\Delta_{2}^{(1)} & =\frac{\omega\left(\overline{a_{11}} \overline{a_{22}}-\overline{a_{12}} \overline{a_{21}}\right)\left(\overline{z^{\prime} f_{1}}-\overline{z^{\prime} f_{2}}\right)+\omega^{\prime}\left(\overline{\bar{a} 21}+\overline{a_{22}}\right) c_{1}-\omega^{\prime}\left(\overline{a_{11}}+\overline{a_{12}}\right) c_{2}}{\omega^{\prime}\left(\overline{a_{11}}+\overline{a_{12}}\right) b_{2}+\omega^{\prime}\left(\overline{a_{21}}+\overline{a_{22}}\right) b_{1}} .
\end{aligned}
$$

Thus all first order quantities are determined. Notice that for the solution (22) to exist, both the denominator in the expression for $\Omega^{(1)}$ and $\omega^{\prime}$ must be nonzero. 


\section{A.2.2 Stability calculations}

We write the monodromy matrix, $M$, for the cluster oscillations in the form

$$
M=\left[\begin{array}{ll}
M_{11} & M_{12} \\
M_{21} & M_{22}
\end{array}\right],
$$

with $2 m_{\nu} \times 2 m_{\mu}$-matrices $M_{\nu \mu}$. Owing to the $\mathbf{S}_{n}$-symmetry, the matrices $M_{\nu \nu}$ are composed of $2 \times 2$ blocks $A^{(\nu)}, B^{(\nu)}$ in the same manner as the monodromy matrix for the uniform oscillation (diagonal blocks formed by $B^{(\nu)}$, all other blocks formed by $A^{(\nu)}$ ), and the $M_{\nu \mu}$ with $\nu \neq \mu$ are composed of single $2 \times 2$ blocks $C^{(\nu)}$.

The eigenvalues of $M$ in the four-dimensional invariant subspace are those of the $4 \times 4$-matrix

$$
K=\left[\begin{array}{cc}
B^{(1)}+\left(m_{1}-1\right) A^{(1)} & m_{2} C^{(1)} \\
m_{1} C^{(2)} & B^{(2)}+\left(m_{2}-1\right) A^{(2)}
\end{array}\right] .
$$

The other eigenvalues of $M$ are those of the $2 \times 2-$ matrices

$$
L^{(\nu)}=B^{(\nu)}-A^{(\nu)},
$$

and are $\left(m_{\nu}-1\right)$-fold degenerate. These eigenvalues govern the stability in directions transverse to the invariant subspace.

At order $O(1)$ the linearized system for phase deviations $\phi_{i}$ and energy deviations $\beta_{i}$ is

$$
\frac{d \phi_{i}}{d t}=\omega^{\prime} \beta_{i}+O(\varepsilon), \quad \frac{d \beta_{i}}{d t}=O(\varepsilon)
$$

which gives

$$
B^{(\nu)}=\left[\begin{array}{cc}
1 & \omega^{\prime} T \\
0 & 1
\end{array}\right]+O(\varepsilon), \quad A^{(\nu)}=O(\varepsilon), \quad C^{\nu)}=O(\varepsilon) .
$$

We first examine the eigenvalues of $L^{(\nu)}$. These matrices have the same asymptotic form as the matrix $L$ for the uniform oscillations, thus we can draw the same conclusions. Letting $L^{(\nu)}=$ $L^{(\nu, 0)}+\varepsilon L^{(\nu, 1)}+O\left(\varepsilon^{2}\right)$, we denote the entries of $L^{(\nu, 1)}$ by $l_{i j}^{(\nu)}$. As in the case of the fully synchronized solution we have to distinguish the following two generic cases:

(a) If $\omega^{\prime} l_{21}^{(\nu)}>0$, then the eigenvalues $\lambda_{1,2}^{(\nu)}$ of $L^{(\nu)}$ are real and given by $\lambda_{1,2}^{(\nu)}=1 \pm \sqrt{\omega^{\prime} T l_{21}^{(\nu)}} \varepsilon+O(\varepsilon)$.

(b) If $\omega^{\prime} l_{21}^{(\nu)}<0$, then the eigenvalues $\lambda_{1,2}^{(\nu)}$ of $L^{(\nu)}$ are complex conjugate with $\left|\lambda_{1,2}^{(\nu)}\right|^{2}=1+l^{(\nu)} \varepsilon+$ $O\left(\varepsilon^{2}\right)$, where $l^{(\nu)}=l_{11}^{(\nu)}+l_{22}^{(\nu)}-\omega^{\prime} T l_{21}^{(\nu)}$.

Notice that, as in Section 2.1.2, passage through isochronicity induces a change in the sign of $\omega^{\prime} T l_{21}^{(\nu)}$.

The relevant $O(\varepsilon)$-corrections of $A^{(\nu)}, B^{(\nu)}, C^{(\nu)}$ are computed in Appendix A.2.3 and lead to the expressions for $l_{21}^{(\nu)}$ and $l^{(\nu)}$ given in equation (29).

Next we examine the eigenvalues of $J=K-I$, where $I$ is the $4 \times 4$ identity matrix. Since $K$ has an eigenvalue one, $J$ has a zero eigenvalue. The cubic equation for the remaining three eigenvalues $\tilde{\lambda}$ has the form,

$$
\tilde{\lambda}^{3}-\left(k_{31} \varepsilon+O\left(\varepsilon^{2}\right)\right) \tilde{\lambda}^{2}-\left(\omega^{\prime} T k_{21} \varepsilon+O\left(\varepsilon^{2}\right)\right) \tilde{\lambda}+\omega^{\prime} T k_{12} \varepsilon^{2}+O\left(\varepsilon^{3}\right)=0,
$$


with certain coefficients $k_{12}, k_{21}, k_{31}$ depending on the first order corrections of the entries of $A^{(\nu)}, B^{(\nu)}, C^{(\nu)}$. The equation for the eigenvalues has a real root of order $O(\varepsilon)$, given by $\tilde{\lambda}=$ $\left(k_{12} / k_{21}\right) \varepsilon+O\left(\varepsilon^{2}\right)$. The two other roots are at leading order given by $\tilde{\lambda}= \pm \sqrt{\omega^{\prime} T k_{21} \varepsilon}+O(\varepsilon)$. If $\omega^{\prime} T k_{21}>0$ they are real and of opposite signs. If $\omega^{\prime} T k_{21}<0$ we need the real part at $O(\varepsilon)$. A simple calculation shows that $2 \operatorname{Re}(\tilde{\lambda})=\left(k_{31}-k_{12} / k_{21}\right) \varepsilon+O\left(\varepsilon^{3 / 2}\right)$. Thus the eigenvalues $\lambda_{1,2,3}$ of $K$ that are different from unity can be characterized as follows:

(1) There is a real eigenvalue $\lambda^{(0)}=1+r \varepsilon+O\left(\varepsilon^{2}\right)$, where $r=k_{12} / k_{21}$.

(2a) If $\omega^{\prime} T k_{21}>0$, then the two other eigenvalues $\lambda_{1,2}$ are real and given by $\lambda_{1,2}=1 \pm \sqrt{\omega^{\prime} T k_{21} \varepsilon}+$ $O(\varepsilon)$. In this case the cluster oscillations always form a saddle.

(2b) If $\omega^{\prime} T k_{21}<0$, then $\lambda_{1,2}$ are complex conjugate with $\left|\lambda_{1,2}\right|^{2}=1+k \varepsilon+O\left(\varepsilon^{3 / 2}\right)$, where $k=k_{31}-\omega^{\prime} T k_{21}-k_{12} / k_{21}$.

By using the $O(\varepsilon)$-corrections $a_{i j}^{(\nu)}, b_{i j}^{(\nu)}, c_{i j}^{(\nu)}$ computed in Appendix A.2.3, the stability coefficients $r, k_{21}, k$ can be represented by averages as follows:

$$
\begin{aligned}
r & =2 \pi \frac{m_{1} \overline{\dot{z} d_{2}} \overline{\left(\dot{z} f_{1}\right)^{\prime}}+m_{2} \overline{\dot{z} d_{1}} \overline{\left(\dot{z} f_{2}\right)^{\prime}}}{m_{1} \dot{\dot{z} d_{2}}+m_{2} \dot{\dot{z} d_{1}}}, \\
k_{21} & =-2 \pi\left(m_{1} \overline{\dot{z} d_{2}}+m_{2} \overline{\dot{z} d_{1}}\right), \\
k & =2 \pi m_{1}\left(\overline{z^{\prime} d_{2}}-\overline{\dot{z} e_{2}}\right)+2 \pi m_{2}\left(\overline{z^{\prime} d_{1}}-\overline{\dot{z} e_{1}}\right)+2 \pi \frac{m_{1} \overline{\dot{z} d_{2}} \overline{\left(\dot{z} f_{2}\right)^{\prime}}+m_{2} \overline{\dot{z} d_{1}} \overline{\left(\dot{z} f_{1}\right)^{\prime}}}{m_{1} \overline{\dot{z} d_{2}}+m_{2} \dot{\dot{z} d_{1}}} .
\end{aligned}
$$

Again the eigenvalues defined through $\left(k_{21}, k\right)$ change their type at isochronicity.

\section{A.2.3 First order terms of the monodromy matrix}

The computation of the relevant $O(\varepsilon)$-terms of the monodromy matrix for the cluster oscillations proceeds analogously to the computation for the uniform oscillation pursued in Appendix A.1.1. We denote the phase-energy variables in the linearized system again by $\left(\phi_{j}, \beta_{j}\right)$, and by $\left(\phi_{j 0}, \beta_{j 0}\right)$ their initial values.

Assume cluster $\nu(=1,2)$ consists of all oscillators with indices in the set $I_{\nu}\left(\left|I_{\nu}\right|=m_{\nu}\right)$, and $\nu \in I_{\nu}$. We decompose $I_{\nu}$ into $I_{\nu}=\{\nu\} \dot{\cup} J_{\nu}$ and examine the linearized equations for $\left(\phi_{\nu}, \beta_{\nu}\right)$. After switching from $t$ to $\varphi$ as independent variable, these equations take the form,

$$
\begin{aligned}
& \omega \dot{\phi}_{\nu}=\omega^{\prime} \beta_{\nu}+\varepsilon\left(a_{1}^{(\nu)} \phi_{\nu}+\tilde{b}_{1}^{(\nu)} \beta_{\nu}+\sum_{j \in J_{\nu}}\left(c_{1}^{(\nu)} \phi_{j}+d_{1}^{(\nu)} \beta_{j}\right)+\sum_{i \in I_{\mu}}\left(e_{1}^{(\nu)} \phi_{i}+f_{1}^{(\nu)} \beta_{i}\right)\right)+O\left(\varepsilon^{2}\right), \\
& \omega \dot{\beta}_{\nu}=\varepsilon\left(a_{2}^{(\nu)} \phi_{\nu}+b_{2}^{(\nu)} \beta_{\nu}+\sum_{j \in J_{\nu}}\left(c_{2}^{(\nu)} \phi_{j}+d_{2}^{(\nu)} \beta_{j}\right)+\sum_{i \in I_{\mu}}\left(e_{2}^{(\nu)} \phi_{i}+f_{2}^{(\nu)} \beta_{i}\right)\right)+O\left(\varepsilon^{2}\right),
\end{aligned}
$$

where $\nu, \mu \in\{1,2\}, \mu \neq \nu$. The $2 \pi$-periodic functions $a_{1,2}^{(\nu)}, b_{1,2}^{(\nu)}, c_{1,2}^{(\nu)}, d_{1,2}^{(\nu)}, e_{1,2}^{(\nu)}$, and $f_{1,2}^{(\nu)}$ follow from the partial derivatives of the phase energy system (6) evaluated at the cluster solution, and $\tilde{b}_{1}^{(\nu)}=b_{1}^{(\nu)}-\Omega^{(1)} \omega^{\prime} / \omega$ (cf. Appendix A.1.1). Expanding the solution according to equation (13), the first order terms $\phi_{\nu}^{(1)}, \beta_{\nu}^{(1)}$ satisfy

$$
\begin{aligned}
\omega \dot{\phi}_{\nu}^{(1)}= & \omega^{\prime} \beta_{\nu}^{(1)}+a_{1}^{(\nu)}\left(\frac{\omega^{\prime}}{\omega} \beta_{\nu 0} \varphi+\phi_{\nu 0}\right)+\tilde{b}_{1}^{(\nu)} \beta_{\nu 0}+c_{1}^{(\nu)} \sum_{j \in J_{\nu}}\left(\frac{\omega^{\prime}}{\omega} \beta_{j 0} \varphi+\phi_{j 0}\right) \\
& +d_{1}^{(\nu)} \sum_{j \in J_{\nu}} \beta_{j 0}+e_{1}^{(\nu)} \sum_{i \in I_{\nu}}\left(\frac{\omega^{\prime}}{\omega} \beta_{i 0} \varphi+\phi_{i 0}\right)+f_{1}^{(\nu)} \sum_{i \in I_{\nu}} \beta_{i 0}, \\
\omega \dot{\beta}_{\nu}^{(1)}= & a_{2}^{(\nu)}\left(\frac{\omega^{\prime}}{\omega} \beta_{\nu 0} \varphi+\phi_{\nu 0}\right)+b_{2}^{(\nu)} \beta_{\nu 0}+c_{2}^{(\nu)} \sum_{j \in J_{\nu}}\left(\frac{\omega^{\prime}}{\omega} \beta_{j 0} \varphi+\phi_{j 0}\right) \\
& +d_{2}^{(\nu)} \sum_{j \in J_{\nu}} \beta_{j 0}+e_{2}^{(\nu)} \sum_{i \in I_{\nu}}\left(\frac{\omega^{\prime}}{\omega} \beta_{i 0} \varphi+\phi_{i 0}\right)+f_{2}^{(\nu)} \sum_{i \in I_{\nu}} \beta_{i 0} .
\end{aligned}
$$


The solution for $\beta_{\nu}^{(1)}$ is found by direct integration. When this solution is evaluated at $\varphi=2 \pi$, we can read off the entries $b_{21}^{(\nu)}, b_{22}^{(\nu)}$ of $B^{(\nu)}$, the entries $a_{21}^{(\nu)}, a_{22}^{(\nu)}$ of $A^{(\nu)}$, and the entries $c_{21}^{(\nu)}, c_{22}^{(\nu)}$ of $C^{(\nu)}$ from the coefficients of $\phi_{\nu 0}, \beta_{\nu 0}$, the coefficients of $\sum_{j \in J_{\nu}} \phi_{j 0}, \sum_{j \in J_{\nu}} \beta_{j 0}$, and the coefficients of $\sum_{i \in I_{\mu}} \phi_{i 0}, \sum_{i \in I_{\mu}} \beta_{i 0}$, respectively. The result is

$$
\begin{aligned}
\omega b_{21}^{(\nu)} & =\int_{0}^{2 \pi} a_{2}^{(\nu)}(\varphi) d \varphi, & \omega b_{22}^{(\nu)} & =\int_{0}^{2 \pi}\left(\frac{\omega^{\prime}}{\omega} a_{2}^{(\nu)}(\varphi) \varphi+b_{2}^{(\nu)}(\varphi)\right) d \varphi, \\
\omega a_{21}^{(\nu)} & =\int_{0}^{2 \pi} c_{2}^{(\nu)}(\varphi) d \varphi, & \omega a_{22}^{(\nu)} & =\int_{0}^{2 \pi}\left(\frac{\omega^{\prime}}{\omega} c_{2}^{(\nu)}(\varphi) \varphi+d_{2}^{(\nu)}(\varphi)\right) d \varphi, \\
\omega c_{21}^{(\nu)} & =\int_{0}^{2 \pi} e_{2}^{(\nu)}(\varphi) d \varphi, & \omega c_{22}^{(\nu)} & =\int_{0}^{2 \pi}\left(\frac{\omega^{\prime}}{\omega} e_{2}^{(\nu)}(\varphi) \varphi+f_{2}^{(\nu)}(\varphi)\right) d \varphi .
\end{aligned}
$$

Similarly the entries $b_{11}^{(\nu)}, a_{11}^{(\nu)}$, and $c_{11}^{(\nu)}$ of $B^{(\nu)}, A^{(\nu)}$, and $C^{(\nu)}$ can be read off from the coefficients of $\phi_{\nu 0}, \sum_{j \in J_{\nu}} \phi_{j 0}$, and $\sum_{i \in I_{\nu}} \phi_{i 0}$ in $\phi_{\nu}^{(1)}(2 \pi)$. After substituting $\beta_{\nu}^{(1)}(\varphi)$ into the $\dot{\phi}_{\nu}^{(1)}$-equation and integrating from 0 to $2 \pi$ we obtain

$$
\begin{aligned}
\omega b_{11}^{(\nu)} & =\frac{\omega^{\prime}}{\omega} \int_{0}^{2 \pi} \int_{0}^{\varphi} a_{2}^{(\nu)}\left(\varphi^{\prime}\right) d \varphi^{\prime} d \varphi+\int_{0}^{2 \pi} a_{1}^{(\nu)}(\varphi) d \varphi \\
\omega a_{11}^{(\nu)} & =\frac{\omega^{\prime}}{\omega} \int_{0}^{2 \pi} \int_{0}^{\varphi} c_{2}^{(\nu)}\left(\varphi^{\prime}\right) d \varphi^{\prime} d \varphi+\int_{0}^{2 \pi} c_{1}^{(\nu)}(\varphi) d \varphi \\
\omega c_{11}^{(\nu)} & =\frac{\omega^{\prime}}{\omega} \int_{0}^{2 \pi} \int_{0}^{\varphi} e_{2}^{(\nu)}\left(\varphi^{\prime}\right) d \varphi^{\prime} d \varphi+\int_{0}^{2 \pi} e_{1}^{(\nu)}(\varphi) d \varphi .
\end{aligned}
$$

Now we represent the functions $a_{1,2}^{(\nu)}, b_{2}^{(\nu)}, c_{1,2}^{(\nu)}, d_{2}^{(\nu)}, e_{1,2}^{(\nu)}$, and $f_{2}^{(\nu)}$, on which the relevant matrix entries depend, in terms of the dissipation and coupling functions. Since the cluster solution is represented by $\Delta / 2$-shifts of $z\left(\varphi, \alpha^{(0)}\right)$ (equation (22)), we denote by $z_{\nu}, \tilde{f}_{\nu}, \tilde{d}_{\nu}, \tilde{e}_{\nu}, \hat{d}_{\nu}$, and $\hat{e}_{\nu}$ the $2 \pi$-periodic functions $z, f_{\nu}, d, e, d_{\nu}$, and $e_{\nu}$ (equations $\left.(24),(28)\right)$ with $\varphi$ replaced by $\varphi+(-1)^{\nu} \Delta / 2$, respectively. With this notation the relevant functions $a_{1,2}^{(\nu)}$ etc. can be written as $(\mu \neq \nu)$

$$
\begin{aligned}
a_{1}^{(\nu)} & =-\omega\left(z_{\nu}^{\prime} \tilde{f}_{\nu}\right)_{, \varphi}+\left(m_{\nu}-1\right) \omega z_{\nu}^{\prime} \tilde{d}_{\nu}+m_{\mu} \omega z_{\nu}^{\prime} \hat{d}_{\nu}, \\
a_{2}^{(\nu)} & =\omega\left(\dot{z}_{\nu} \tilde{f}_{\nu}\right)_{, \varphi}-\left(m_{\nu}-1\right) \omega \dot{z}_{\nu} \tilde{d}_{\nu}-m_{\mu} \omega \dot{z}_{\nu} \hat{d}_{\nu}, \\
b_{2}^{(\nu)} & =\left(\omega \dot{z}_{\nu} \tilde{f}_{\nu}\right)^{\prime}-\left(m_{\nu}-1\right) \omega \dot{z}_{\nu} \tilde{e}_{\nu}-m_{\mu} \omega \dot{z}_{\nu} \hat{e}_{\nu},
\end{aligned}
$$

and

$$
\begin{aligned}
c_{1}^{(\nu)} & =-\omega z_{\nu}^{\prime} \tilde{d}_{\nu}, & e_{1}^{(\nu)} & =-\omega z_{\nu}^{\prime} \hat{d}_{\nu}, \\
c_{2}^{(\nu)} & =\omega \dot{z}_{\nu} \tilde{d}_{\nu}, & e_{2}^{(\nu)} & =\omega \dot{z}_{\nu} \hat{d}_{\nu}, \\
d_{2}^{(\nu)} & =\omega \dot{z}_{\nu} \tilde{e}_{\nu}, & f_{2}^{(\nu)} & =\omega \dot{z}_{\nu} \hat{e}_{\nu} .
\end{aligned}
$$

By substituting these expressions into the integrals above, reversing the phase shift, and performing some integrations by parts, the relevant matrix entries can be represented by averages over $2 \pi-$ periodic functions as follows:

$$
\begin{aligned}
a_{11}^{(\nu)} & =-2 \pi \overline{z^{\prime} d}+\omega^{\prime} T\left(\pi \overline{\dot{z} d}+\overline{D_{\nu 0}}\right) \\
a_{21}^{(\nu)} & =2 \pi \overline{\dot{z} d} \\
a_{22}^{(\nu)} & =2 \pi \overline{\dot{z} e}+\omega^{\prime} T\left(\pi \overline{\dot{z} d}-\overline{D_{\nu 0}}\right) \\
b_{11}^{(\nu)} & =2 \pi\left(m_{\nu}-1\right) \overline{z^{\prime} d}+2 \pi m_{\mu} \overline{z^{\prime} d_{\nu}}-\omega^{\prime} T\left(m_{\nu}-1\right)\left(\pi \overline{\dot{z} d}+\overline{D_{\nu 0}}\right)
\end{aligned}
$$




$$
\begin{aligned}
& -\omega^{\prime} T m_{\mu}\left(\pi \overline{\dot{z} d_{\nu}}+\overline{D_{\nu}}\right)-\left.\omega^{\prime} T\left(\dot{z} f_{\nu}\right)\right|_{\varphi=\varphi_{\nu}} \\
b_{21}^{(\nu)}= & -2 \pi\left(m_{\nu}-1\right) \overline{\dot{z} d}-2 \pi m_{\mu} \overline{\dot{z} d_{\nu}} \\
b_{22}^{(\nu)}= & 2 \pi \overline{\left(\dot{z} f_{\nu}\right)^{\prime}}-2 \pi\left(m_{\nu}-1\right) \overline{\dot{z} e}-2 \pi m_{\mu} \overline{\dot{z} e_{\nu}}-\omega^{\prime} T\left(m_{\nu}-1\right)\left(\pi \overline{\dot{z} d}-\overline{D_{\nu 0}}\right) \\
& -\omega^{\prime} T m_{\mu}\left(\pi \overline{\dot{z} d_{\nu}}-\overline{D_{\nu}}\right)+\left.\omega^{\prime} T\left(\dot{z} f_{\nu}\right)\right|_{\varphi=\varphi_{\nu}} \\
c_{11}^{(\nu)}= & -2 \pi \overline{z^{\prime} d_{\nu}}+\omega^{\prime} T\left(\pi \overline{\dot{z} d_{\nu}}+\overline{D_{\nu}}\right) \\
c_{21}^{(\nu)}= & 2 \pi \overline{\dot{z} d_{\nu}} \\
c_{22}^{(\nu)}= & 2 \pi \overline{\dot{z} e_{\nu}}+\omega^{\prime} T\left(\pi \overline{\dot{z} d_{\nu}}-\overline{D_{\nu}}\right),
\end{aligned}
$$

where $\mu \neq \nu, \varphi_{\nu}=(-1)^{\nu} \Delta / 2$, functions $d, e, d_{\nu}, e_{\nu}$ are as defined in equation (24), and

$$
D_{\nu 0}=\int_{\varphi_{\nu}}^{\varphi+\varphi_{\nu}}(\dot{z} d-\overline{\dot{z}} d) d \varphi, \quad D_{\nu}=\int_{\varphi_{\nu}}^{\varphi+\varphi_{\nu}}\left(\dot{z} d_{\nu}-\overline{\dot{z} d_{\nu}}\right) d \varphi
$$

The expressions for $l_{21}^{(\nu)}, l^{(\nu)}$ follow directly from the above average representations of the matrix entries.

The coefficients that occur in the characteristic equation (A.4) are:

$$
\begin{aligned}
k_{12} & =\sum_{\nu=1}^{2}\left(\tilde{b}_{21}^{(\nu)}\left(\tilde{b}_{11}^{(\mu)}+\tilde{b}_{22}^{(\mu)}\right)-m_{1} m_{2} c_{21}^{(\nu)}\left(c_{11}^{(\mu)}+c_{22}^{(\mu)}\right)\right) \\
k_{21} & =\tilde{b}_{21}^{(1)}+\tilde{b}_{21}^{(2)} \\
k_{31} & =\tilde{b}_{11}^{(1)}+\tilde{b}_{22}^{(1)}+\tilde{b}_{11}^{(2)}+\tilde{b}_{22}^{(2)},
\end{aligned}
$$

where $\mu \neq \nu$, and

$$
\tilde{b}_{i j}^{(\nu)}=b_{i j}^{(\nu)}+\left(m_{\nu}-1\right) a_{i j}^{(\nu)} .
$$

Substitution of the matrix entries above then leads to the expressions (A.5) for $r=k_{12} / k_{21}, k_{21}$, $k=k_{31}-\omega^{\prime} T k_{21}-r$.

\section{A.3 Persistence conditions and stability coefficients for the system (36)}

Basic solutions The persistence conditions for the basic solutions $U O, R W, O P C$ (arbitrary $\left.c_{1}, c_{2}, c_{3}\right)$, and $O P C 13\left(c_{2}=0\right)$ are

$$
\begin{aligned}
U O & : p_{0}=0, \\
R W & : p_{0}+c_{2}\left(\overline{\dot{z}\left(\dot{z}_{\pi}-\dot{z}\right)}+{\overline{\dot{z}}\left(\dot{z}_{\pi / 2}+\dot{z}_{-\pi / 2}-2 \dot{z}\right)}\right)=0, \\
O P C & : p_{0}+2 c_{2} \overline{\dot{z}\left(\dot{z}_{\pi}-\dot{z}\right)}=0, \\
O P C 13 & : p_{0}=0 \quad\left(c_{2}=0\right),
\end{aligned}
$$

where

$$
p_{0}=s \overline{\dot{z}^{2}}-\overline{\dot{z}^{2}(z-2)^{2}},
$$

and $z_{\Delta}$ denotes the phase shifted function $z_{\Delta}(\varphi)=z(\varphi+\Delta)$. Note that these persistence conditions are identical if $c_{2}=0$.

We now summarize in turn the stability coefficients for $U O, R W, O P C$, and $O P C 13$.

Uniform oscillation UO:

$$
l_{21}=-8 \pi c_{1} \overline{\dot{z}^{2}}, \quad l=l_{0}-4 c_{2} T, \quad r=l_{0},
$$


where

$$
l_{0}=-4 \pi \omega \overline{\dot{z} \dot{z}^{\prime}\left((z-2)^{2}-s\right)}-4 \pi \omega \overline{z^{\prime} \dot{z}^{2}(z-2)} .
$$

Numerical computation shows that $l_{0}<0$ when evaluated along the solution of the $U O$-persistence condition $p_{0}=0$.

Rotating wave oscillation $R W$ : We set

$$
\dot{z}_{ \pm}=\dot{z}_{\pi / 2} \pm \dot{z}_{-\pi / 2}, \quad \dot{z}_{3, \pi}=\dot{z}_{\pi}\left(z_{\pi}-z\right)^{2}, \quad \dot{z}_{3, \pm}=\dot{z}_{\pi / 2}\left(z_{\pi / 2}-z\right)^{2} \pm \dot{z}_{-\pi / 2}\left(z_{-\pi / 2}-z\right)^{2} .
$$

With this notation the stability coefficients for $R W$ can be written as

$$
\begin{aligned}
l_{21}^{(1,3)} & =-2 \pi c_{1} \overline{\dot{z}\left(\dot{z}_{+}+2 \dot{z}_{\pi}\right)}-6 \pi c_{3}\left(\overline{\dot{z} \dot{z}_{3,+}}+2 \overline{\dot{z} \dot{z} \dot{z}_{3, \pi}}\right) \pm 2 \pi i \omega c_{2} \overline{\dot{z} \dot{z}_{-}}, \\
l^{(1,3)} & =l_{0}-2 \pi c_{2}\left(\omega^{\prime} \overline{\dot{z}\left(\dot{z}_{+}+2 \dot{z}_{\pi}\right)}+2 \omega \overline{\dot{z}^{\prime}\left(\dot{z}_{\pi}+3 \dot{z}\right)}\right) \pm 4 \pi i\left(c_{1} \overline{z^{\prime} \dot{z}_{-}}+3 c_{3} \overline{z^{\prime} \dot{z}_{3,-}}\right), \\
l_{21}^{(2)} & =-4 \pi c_{1} \overline{\dot{z} \dot{z}_{+}}-12 \pi c_{3} \overline{\dot{z} \dot{z}_{3,+}}, \\
l^{(2)} & =l_{0}-4 \pi c_{2}\left(\omega^{\prime} \overline{\dot{z} \dot{z}_{+}}+\omega \overline{\dot{z}^{\prime}\left(\dot{z}_{+}+3 \dot{z}-\dot{z}_{\pi}\right)}\right), \\
r & =l_{0}+4 \pi \omega c_{2} \overline{\dot{z}^{\prime}\left(\dot{z}_{+}+\dot{z}_{\pi}-3 \dot{z}\right)} .
\end{aligned}
$$

Since $\dot{z}$ is odd, we have $\overline{\dot{z} \dot{z}_{\pi}}<0$ and $\overline{\dot{z} \dot{z}_{3, \pi}}<0$. Numerical inspection shows that the averages multiplying $c_{1}$ and $c_{3}$ in $l_{21}^{(1,3)}$ are both negative, and the corresponding averages in $l_{21}^{(2)}$ are negative and positive, respectively.

Out of phase cluster oscillation OPC:

$$
\begin{aligned}
l_{21} & =-4 \pi c_{1} \overline{\dot{z}\left(\dot{z}+\dot{z}_{\pi}\right)}-12 \pi c_{3} \overline{\dot{z} \dot{z}_{3, \pi}}, \\
l & =l_{0}-2 c_{2} T-4 \pi c_{2}\left(\omega^{\prime} \overline{\dot{z}} \dot{z}_{\pi}+2 \omega \overline{\dot{z}^{\prime} \dot{z}}\right), \\
k_{21} & =-8 \pi \overline{\dot{z}} \dot{z}_{\pi}-24 \pi c_{3} \overline{\dot{z} \dot{z}_{3, \pi}}, \\
k & =l_{0}-8 \pi c_{2}\left(\omega^{\prime} \dot{z} \dot{z} \dot{z}_{\pi}+\omega \dot{\dot{z}^{\prime}\left(\dot{z}+\dot{z}_{\pi}\right)}\right), \\
r & =l_{0}+8 \pi \omega c_{2} \overline{\dot{z}^{\prime}\left(\dot{z}_{\pi}-\dot{z}\right)} .
\end{aligned}
$$

Out of phase cluster oscillation OPC13 $\left(c_{2}=0\right)$ :

$$
\begin{gathered}
l_{21}=-2 \pi c_{1} \overline{\dot{z}\left(3 \dot{z}+\dot{z}_{\pi}\right)}-6 \pi c_{3} \overline{\dot{z} \dot{z}_{\pi}\left(z_{\pi}-z\right)^{2}}, \\
k_{21}=-8 \pi c_{1} \overline{\dot{z} \dot{z} \dot{z}_{\pi}}-24 \pi c_{3} \overline{\dot{z} \dot{z}_{\pi}\left(z_{\pi}-z\right)^{2}}, \\
l=k=r=l_{0} .
\end{gathered}
$$

Notice that the two averages in $l_{21}$ have opposite signs, and in $k_{21}$ they have equal signs.

Cluster oscillations The two persistence conditions for $C_{\varepsilon}(2,2)$ and $C_{\varepsilon}(1,3)$ can be rewritten in the form

$$
\begin{aligned}
& C_{\varepsilon}(2,2): \quad c_{1} \overline{\dot{z}\left(z_{\Delta}-z_{-\Delta}\right)}+c_{3} \overline{\dot{z}\left(\left(z_{\Delta}-z\right)^{3}-\left(z_{-\Delta}-z\right)^{3}\right)}=0, \\
& \overline{\dot{z}^{2}\left(s-(z-2)^{2}\right)}+c_{2} \overline{\dot{z}\left(\dot{z}_{\Delta}+\dot{z}_{-\Delta}-2 \dot{z}\right)}=0 \text {, } \\
& C_{\varepsilon}(1,3): \quad c_{1} \overline{\dot{z}\left(3 z_{\Delta}-z_{-\Delta}\right)}+c_{3}\left(\overline{3 \dot{z}\left(z_{\Delta}-z\right)^{3}-\dot{z}\left(z_{-\Delta}-z\right)^{3}}\right)+\omega c_{2} \overline{\dot{z}\left(3 \dot{z}_{\Delta}-\dot{z}_{-\Delta}-2 \dot{z}\right)}=0 \text {, } \\
& \overline{\dot{z}^{2}\left(s-(z-2)^{2}\right)}+\left(3 c_{2} / 4\right) \overline{\dot{z}\left(\dot{z}_{\Delta}+\dot{z}_{-\Delta}-2 \dot{z}\right)}=0 \text {. }
\end{aligned}
$$


The second equation for $C_{\varepsilon}(2,2)$ does not depend on $c_{2}$ because $\overline{\dot{z}\left(\dot{z}_{\Delta}-\dot{z}_{-\Delta}\right)}=0$.

If $c_{2}=0$, the second equation in both cases coincides with the persistence condition for the basic solutions and so defines a segment of the persistence graph of Figure 2 (a). In this case $\Delta=\pi$ is a distinguished solution of the first equation also for $C_{\varepsilon}(1,3)$ because $z_{\pi}$ is even. Moreover, since

$$
g(\Delta, \alpha)=c_{1} \overline{\dot{z} z_{\Delta}}+c_{3} \overline{\dot{z}\left(z_{\Delta}-z\right)^{3}}
$$

is an odd function of $\Delta$, the first persistence condition for both $C_{\varepsilon}(1,3)$ and $C_{\varepsilon}(2,2)$ reduces to the single equation $g(\Delta, \alpha)=0$, hence the two branches are identical.

The transverse stability coefficients and the coefficient $k_{21}$ are

$$
\begin{aligned}
l_{21}^{( \pm)} & =-4 \pi c_{1} \overline{\dot{z}\left(\dot{z}+\dot{z}_{ \pm \Delta}\right)}-12 \pi c_{3} \overline{\dot{z} \dot{z}_{ \pm \Delta}\left(z_{ \pm \Delta}-z\right)^{2}}-4 \pi \omega c_{2} \bar{z}_{\ddot{z}_{ \pm \Delta}} \\
l^{( \pm)} & =l_{0}-2 c_{2} T-8 \pi \omega c_{2} \overline{\dot{z}} \dot{z}^{\prime}-4 \pi \omega^{\prime} c_{2} \overline{\dot{z}} \dot{z}_{ \pm \Delta}, \\
k_{21} & =-4 \pi c_{1} \overline{\dot{z}\left(\dot{z}_{\Delta}+\dot{z}_{-\Delta}\right)}-12 \pi c_{3} \dot{\dot{z}\left(\dot{z}_{\Delta}\left(z_{\Delta}-z\right)^{2}+\dot{z}_{-\Delta}\left(z_{-\Delta}-z\right)^{2}\right)}
\end{aligned}
$$

for $C_{\varepsilon}(2,2)$, and

$$
\begin{aligned}
l_{21} & =-2 \pi c_{1} \overline{\dot{z}\left(3 \dot{z}+\dot{z}_{-\Delta}\right)}-6 \pi c_{3} \overline{\dot{z} \dot{z}-\Delta\left(z_{-\Delta}-z\right)^{2}}-2 \pi \omega c_{2} \overline{\dot{z} \ddot{z}_{-\Delta}}, \\
l & =l_{0}-3 c_{2} T-4 \pi \omega c_{2} \overline{\dot{z}} \dot{z}^{\prime}+\pi \omega^{\prime} c_{2} \overline{\dot{z}^{2}}-(3 \pi / 2) \omega^{\prime} c_{2} \overline{\dot{z}\left(\dot{z}_{\Delta}+\dot{z}_{-\Delta}\right)}, \\
k_{21} & =-2 \pi c_{1} \overline{\dot{z}\left(3 \dot{z}_{\Delta}+\dot{z}_{-\Delta}\right)}-6 \pi c_{3} \overline{\dot{z}\left(3 \dot{z}_{\Delta}\left(z_{\Delta}-z\right)^{2}+\dot{z}_{-\Delta}\left(z_{-\Delta}-z\right)^{2}\right)}-2 \pi \omega c_{2} \overline{\dot{z}\left(3 \ddot{z}_{\Delta}+\ddot{z}_{-\Delta}\right)},
\end{aligned}
$$

for $C_{\varepsilon}(1,3)$. The two remaining coefficients $k, r$ are messy and are not written down explicitly. We refer to Appendix A.2 for their general forms. Numerically we observe that $k, l^{( \pm)}, l, r<0$ for the parameter ranges we have studied.

Cluster oscillations $C_{1}(2,2)$ and $C_{1}(1,3)$ : To present the persistence conditions for cluster oscillations with $O(1)$ energy differences, we introduce the function $y(\varphi, \alpha)=z(\varphi, h(\alpha))$, where $\beta=h(\alpha)$ defines the nontrivial branch of the $1: 1$ resonance set, $\omega(\alpha)=\omega(h(\alpha))$. With this notation the two persistence conditions for $C_{1}\left(m_{1}, m_{2}\right)$ can be written as

$$
s \omega \overline{\dot{z}^{2}}-f=0, \quad s \omega \overline{\dot{y}^{2}}-g=0,
$$

where

$$
\begin{aligned}
& f=\omega \overline{\dot{z}^{2}(z-2)^{2}}-m_{1} c_{1} \overline{\dot{z}\left(y_{\Delta}-z\right)}-m_{1} c_{3} \overline{\dot{z}\left(y_{\Delta}-z\right)^{3}}-m_{1} \omega c_{2} \overline{\dot{z}\left(\dot{y}_{\Delta}-\dot{z}\right)}, \\
& g=\omega \overline{\dot{y}^{2}(y-2)^{2}}-m_{2} c_{1} \overline{\dot{y}\left(z_{-\Delta}-y\right)}-m_{2} c_{3} \overline{\dot{y}\left(z_{-\Delta}-y\right)^{3}}-m_{2} \omega c_{2} \overline{\dot{y}\left(\dot{z}_{-\Delta}-\dot{y}\right)} .
\end{aligned}
$$

The solution branches shown in Sections 3.2 and 3.3 have been calculated by solving the equation $\overline{\dot{y}^{2}} f-\overline{\dot{z}^{2}} g=0$ for $\Delta(\alpha)$ and then computing $s(\alpha)=f / \omega \overline{\dot{z}^{2}}$. 